\title{
Fivebrane instantons, topological wave functions and hypermultiplet moduli spaces
}

\author{
Sergei Alexandrov, ${ }^{a}$ Daniel Persson ${ }^{b}$ and Boris Pioline ${ }^{c}$ \\ ${ }^{a}$ Laboratoire de Physique Théorique \& Astroparticules, CNRS UMR 520\%, \\ Université Montpellier II, \\ 34095 Montpellier Cedex 05, France \\ ${ }^{b}$ Institut für Theoretische Physik, ETH Zürich, \\ CH-8093 Zürich, Switzerland \\ ${ }^{c}$ Laboratoire de Physique Théorique et Hautes Energies, CNRS UMR 7589, \\ Université Pierre et Marie Curie - Paris 6, \\ 4 place Jussieu, 75252 Paris Cedex 05, France \\ E-mail: sergey.alexandrov@univ-montp2.fr, \\ daniel.persson@itp.phys.ethz.ch, pioline@lpthe.jussieu.fr
}

ABSTRACT: We investigate quantum corrections to the hypermultiplet moduli space $\mathcal{M}$ in Calabi-Yau compactifications of type II string theories, with particular emphasis on instanton effects from Euclidean NS5-branes. Based on the consistency of D- and NS5instanton corrections, we determine the topology of the hypermultiplet moduli space at fixed string coupling, as previewed in [1]. On the type IIB side, we compute corrections from $(p, k)$-fivebrane instantons to the metric on $\mathcal{M}$ (specifically, the correction to the complex contact structure on its twistor space $\mathcal{Z}$ ) by applying S-duality to the D-instanton sum. For fixed fivebrane charge $k$, the corrections can be written as a non-Gaussian theta series, whose summand for $k=1$ reduces to the topological A-model amplitude. By mirror symmetry, instanton corrections induced from the chiral type IIA NS5-brane are similarly governed by the wave function of the topological B-model. In the course of this investigation we clarify charge quantization for coherent sheaves and find hitherto unnoticed corrections to the Heisenberg, monodromy and S-duality actions on $\mathcal{M}$, as well as to the mirror map for Ramond-Ramond fields and D-brane charges.

KEYwords: Nonperturbative Effects, Discrete and Finite Symmetries, Topological Strings, String Duality

ARXIV EPRINT: 1010.5792 


\section{Contents}

1 Introduction and summary 1

1.1 The hypermultiplet moduli space 2

1.2 Sum over $H$-flux and Gaussian theta series 3

1.3 NS5-brane partition function and non-Gaussian theta series 4

1.4 Topology of the hypermultiplet moduli space and quantum corrections 6

1.5 Quantum mirror symmetry, S-duality and $(p, k)$ fivebranes 7

$\begin{array}{lll}1.6 & \text { Fivebrane partition function and twistors } & 9\end{array}$

$\begin{array}{lll}1.7 & \text { Outline } & 9\end{array}$

2 Flux partition functions and topological wave functions 10

$\begin{array}{ll}2.1 \text { Setting the stage } & 10\end{array}$

$\begin{array}{lll}2.2 & \text { Factorizing the non-chiral partition function } & 13\end{array}$

$\begin{array}{lll}2.3 & \text { Chiral partition function and Gaussian theta series } & 16\end{array}$

$\begin{array}{lll}2.4 & \text { Non-Gaussian theta series and wave-functions } & 18\end{array}$

$\begin{array}{ll}2.5 \text { Topological wave functions in different polarizations } & 19\end{array}$

$\begin{array}{lll}2.5 .1 & \text { Griffiths polarization } & 19\end{array}$

$\begin{array}{lll}2.5 .2 & \text { Weil polarization } & 21\end{array}$

$\begin{array}{ll}2.5 .3 & \text { Real polarization and intertwiners } \\ 2.5 .4 & 22\end{array}$

2.5.4 Holomorphic topological partition function 23

2.5.5 Topological A-model and Donaldson-Thomas invariants 24

3 Topology of the type IIA hypermultiplet moduli space $\quad \mathbf{2 5}$

$\begin{array}{ll}3.1 & \text { Perturbative HM moduli space } \\ & 3.16\end{array}$

3.1.1 Ten-dimensional origin of the one-loop correction 27

$\begin{array}{lll}3.2 & \text { Topology and instanton corrections } & 27\end{array}$

3.2.1 Instanton contributions to the HM moduli space 28

$\begin{array}{ll}3.2 .2 & \text { Large gauge transformations } \\ 3.2 .3 & \text { Mon }\end{array}$

$\begin{array}{lll}3.2 .3 & \text { Monodromies } & 30\end{array}$

4 Mirror symmetry, S-duality and NS5-branes in type IIB 32

4.1 Topology of the perturbative type IIB HM moduli space 33

4.1.1 Kähler moduli space $\quad 33$

$\begin{array}{lll}\text { 4.1.2 Charge quantization } & 34\end{array}$

4.1.3 Symplectic Jacobian and mirror symmetry 36

4.1.4 Monodromy around the large volume point 37

$\begin{array}{lll}4.2 & \text { S-duality } & 38\end{array}$

$\begin{array}{lll}4.3 & \text { Semi-classical }(p, k) 5 \text {-brane instantons } & 40\end{array}$ 
5 NS5-instantons in twistor space

5.1 Twistor techniques 43

$\begin{array}{lll}5.2 & \text { Twistorial description of } \mathcal{M} & 45\end{array}$

5.2.1 Perturbative twistor space $\quad 45$

$\begin{array}{lll}5.2 .2 & \text { D-instanton corrected twistor space } & 46\end{array}$

$\begin{array}{lll}5.2 .3 & \text { S-duality in twistor space } & 48\end{array}$

$\begin{array}{lll}5.3 & \text { Contact structure for fivebrane instantons } & 49\end{array}$

5.4 Poincaré series for NS5-instantons and the topological string amplitude 51

5.5 Fivebrane partition function from twistor space 53

$\begin{array}{lll}\text { 5.5.1 Penrose transform } & 54\end{array}$

5.5.2 Non-Gaussian fivebrane partition function $\quad 56$

$\begin{array}{llr}6 & \text { Discussion } & 58\end{array}$

A Action of Heisenberg shifts and monodromies on $\boldsymbol{H}_{k, p, \hat{\gamma}} \quad 60$

$\begin{array}{lll}\text { A.1 Heisenberg symmetry } & 61\end{array}$

A.2 Monodromy transformations $\quad 63$

B List of notations $\quad 64$

\section{Introduction and summary}

To date, the hypermultiplet (HM) moduli space $\mathcal{M}$ in type II string theories compactified on a Calabi-Yau (CY) threefold $\mathcal{X}$ has remained poorly understood. Yet, its importance can hardly be overstated: quantum corrections to the moduli space metric encode important geometric invariants of $\mathcal{X}$, which are closely related to the degeneracies of BPS black holes [2]. Moreover, this encoding naturally incorporates chamber dependence and duality invariance. Obtaining the exact moduli space metric on $\mathcal{M}$ would have far-reaching implications for mirror symmetry, and provide incisive tests of string dualities. It may also be relevant for model building, as many $\mathcal{N}=1$ string vacua can be obtained by gauging isometries on the hypermultiplet branch.

Quantum corrections to the tree-level metric on $\mathcal{M}$ fall in three categories: perturbative, D-brane instantons and NS5-brane instantons [3]. Each of these corrections must preserve the quaternion-Kähler property of the metric, as required for supersymmetry [4]. While the perturbative and D-instanton corrections are by now fairly well understood, NS5brane instantons have remained elusive. One technical reason is that fivebrane instantons break all continuous isometries, and their implementation consistently with supersymmetry necessitates the full machinery of twistor techniques for quaternion-Kähler manifolds. Another deeper reason is that the type IIA fivebrane supports a chiral worldvolume theory [5], the partition function of which is notoriously subtle to define. On the type IIB side, the world-volume theory of the NS5-brane is non-chiral [5] and follows in principle 
by S-duality from that of the D5-brane, but S-duality is also notoriously subtle in vacua with $\mathcal{N}=2$ supersymmetry. In this paper, we take steps towards constructing fivebrane instanton corrections on both sides, using insights from topological strings, mirror symmetry and S-duality. The remainder of the introduction is devoted to an account of the relevant background for the paper, while concurrently providing a concise summary of our main results.

\subsection{The hypermultiplet moduli space}

At tree level, the quaternion-Kähler metric on the HM moduli space $\mathcal{M}$ in type IIA (respectively, type IIB) string theory compactified on $\mathcal{X}$ is obtained by the so-called $c$-map procedure from the special Kähler metric on the vector multiplet (VM) moduli space of type IIB (respectively, type IIA) string theory compactified on the same CY threefold $\mathcal{X}[6,7]$. It describes the vacuum expectation values and couplings of the dilaton $e^{\phi} \sim 1 / g_{(4)}^{2}$, the complex structure (respectively, complexified Kähler) moduli $z^{a}$, the periods $\left(\zeta^{\Lambda}, \tilde{\zeta}_{\Lambda}\right)$ of the Ramond-Ramond (RR) fields on $\mathcal{X}$, and notably the Neveu-Schwarz (NS) axion $\sigma$, dual to the Kalb-Ramond field $B$ in four dimensions (the range and meaning of the indices $a$ and $\Lambda$ will be specified below). At fixed, vanishingly small string coupling $g_{s}$ and ignoring the NS-axion (i.e., modding out by translations along $\sigma$, which are symmetries of the perturbative theory), the "reduced" HM moduli space in type IIA string theory is known to be topologically the intermediate Jacobian ${ }^{1} \mathcal{J}_{c}(\mathcal{X})$ of the CY family $\mathcal{X}$, which is a torus bundle over the complex structure moduli space $\mathcal{M}_{c}(\mathcal{X})$ with fiber $\mathcal{T} \equiv H^{3}(\mathcal{X}, \mathbb{R}) / H^{3}(\mathcal{X}, \mathbb{Z})$, which parametrizes the space of harmonic RR three-form fields $C$ over $\mathcal{X}$ modulo large gauge transformations [8]. In type IIB, the "reduced" HM moduli space is similarly a torus bundle over the complexified Kähler moduli space $\mathcal{M}_{K}(\mathcal{X})$, the fiber of which is the quotient $\mathcal{T}=H^{\text {even }}(\mathcal{X}) / \Gamma$ where $\Gamma$ is the lattice of D-brane charges. As in type IIA, $\mathcal{T}$ parametrizes the space of harmonic $\mathrm{RR}$ fields over $\mathcal{X}$, modulo large gauge transformations. We shall refer to the total space of this torus bundle as the symplectic Jacobian $\mathcal{J}_{K}(\mathcal{X})$. In type IIA (respectively, IIB), the NS-axion $\sigma$ parametrizes a circle bundle $\mathcal{C}$ over $\mathcal{J}_{c}(\mathcal{X})$ (respectively, $\mathcal{J}_{K}(\mathcal{X})$ ), the topology of which has remained hitherto obscure. One of our goals will be to clarify the topology of the circle bundle $\mathcal{C}$, by analyzing the breaking of translational isometries along $\sigma$ due to fivebrane instantons.

The quantum corrected quaternion-Kähler metric on $\mathcal{M}$ is most conveniently described in terms of the twistor space $\mathcal{Z}_{\mathcal{M}}$ associated to $\mathcal{M}$, a complex contact manifold locally of the form $\mathbb{C} P^{1} \times \mathcal{M}$ (see e.g. [9-12]). By the LeBrun-Salamon theorem, the quaternionKähler metric on $\mathcal{M}$ can be recovered from the complex contact structure on $\mathcal{Z}$, and linear perturbations of $\mathcal{M}$ respecting the quaternion-Kähler property are encoded in holomorphic sections of the Čech cohomology group $H^{1}\left(\mathcal{Z}_{\mathcal{M}}, \mathcal{O}(2)\right)$. The one-loop correction to the treelevel metric was computed and further studied in [13-17]. It is believed to exhaust the series of perturbative corrections, in the sense that higher loop corrections are expected to be removable by field redefinitions. The twistorial description of the one-loop correction was

\footnotetext{
${ }^{1}$ The intermediate Jacobian usually refers to the torus $\mathcal{T}$ itself, rather than the total space of the fiber bundle over $\mathcal{M}_{c}(\mathcal{X})$. However, since $\mathcal{X}$ is really a family of CY manifolds with varying complex structure, we find this language convenient.
} 
found in [12], as a logarithmic singularity of a certain canonical Darboux coordinate at the north and south poles of $\mathbb{C} P^{1}$.

The D-instanton corrections to the complex contact structure of $\mathcal{Z}_{\mathcal{M}}$ (and, therefore, to the quaternion-Kähler metric on $\mathcal{M}$ ) were derived in [18-20], building on earlier work [17, 21-23]. Key ingredients in this derivation were S-duality, electric-magnetic duality (i.e. monodromy invariance) and mirror symmetry. In the type IIA language, these D-instanton corrections are parametrized by a chamber-dependent "instanton measure" $n_{\gamma}(z)$, which counts the number of stable special Lagrangian (sLag) submanifolds of $\mathcal{X}$ in the homology class $\gamma \in H_{3}(\mathcal{X}, \mathbb{Z})$, on which Euclidean D2-branes can be wrapped. The resulting complex contact structure is closely analogous to the complex symplectic structure found on the twistor space of the (hyperkähler) HM moduli space in $\mathcal{N}=2$ super-Yang Mills theories on $\mathbb{R}^{3} \times S^{1}$ [24]. Indeed, upon compactifying the type IIA string theory further on a circle and applying T-duality, $\mathcal{M}$ is mapped to the VM moduli space of the dual type IIB string theory in 3 dimensions, thus providing a string theory analog of the set-up studied in [24].

Similarly as in $\mathcal{N}=2$ field theories [24], the global consistency of the complex contact structure on $\mathcal{Z}_{\mathcal{M}}$ requires that the instanton measure $n_{\gamma}(z)$ satisfies certain wall-crossing constraints, identical to the constraints for the generalized Donaldson-Thomas (DT) invariants established in $[25,26]$. This supports the expectation that the instanton measure $n_{\gamma}(z)$ is in fact equal to the generalized DT invariant $\Omega(\gamma, z)$ defined in $[25,26]$, and also to the indexed degeneracy of $4 \mathrm{D}$ black holes in type IIB string theory on $\mathcal{X}$ in a vacuum with asymptotic values of the VM moduli determined by $z^{a}$. In the following we shall therefore identify these objects and denote them by the common symbol $\Omega(\gamma, z)$, sometimes omitting the dependence on the moduli $z^{a}$. Note however that due to the exponential growth of the degeneracies of BPS black holes, D-instanton corrections only make sense as an asymptotic series. The ambiguity of this asymptotic series can be argued to be of the order of the corrections expected from Kaluza-Klein monopoles in type IIB on $\mathcal{X} \times S^{1}$, or after T-duality, NS5-branes in type IIA on $\mathcal{X}[27]$.

\subsection{Sum over $H$-flux and Gaussian theta series}

In contrast to D-instantons, fivebrane instantons have remained largely mysterious. The contribution of one Euclidean type IIA NS5-brane wrapped on $\mathcal{X}$ (or one M5-brane in the M-theory picture) is expected to involve a sum over harmonic configurations $H \in \Gamma=$ $H^{3}(\mathcal{X}, \mathbb{Z})$ of the 3 -form flux $H$ supported on the fivebrane worldvolume. Equivalently, one expects contributions from all D2-NS5 (or M2-M5) bound states, where the D2/M2-brane can wrap any sLag submanifold ${ }^{2} \gamma \in \Gamma$ of $\mathcal{X}$. However, the self-duality constraint on $H$ implies that fluxes on three-cycles $\gamma, \gamma^{\prime}$ with non-zero intersection product $\left\langle\gamma, \gamma^{\prime}\right\rangle$ cannot be measured (nor defined) simultaneously, and so the sum should run over a Lagrangian sublattice $\Gamma_{e} \subset \Gamma$ only [28] (see also [29-36]). As we review in section 2, the partition function $\mathcal{Z}_{\mathrm{G}}^{(1)}$ for a Gaussian self-dual three-form on $\mathcal{X}$ is then a holomorphic section of a

\footnotetext{
${ }^{2}$ Up to torsion, the lattices $\Gamma=H^{3}(\mathcal{X}, \mathbb{Z})$ and $\Gamma^{*}=H_{3}(\mathcal{X}, \mathbb{Z})$ are isomorphic by Poincaré duality. In this work we shall ignore torsion and identify $\Gamma=\Gamma^{*}$.
} 
certain line bundle $\mathcal{L}_{\Theta}$ over the intermediate Jacobian torus, which we shall call the "theta line bundle". The first Chern class of $\mathcal{L}_{\Theta}$ is known to be equal to the Kähler class of $\mathcal{T}$ [28]:

$$
\left.c_{1}\left(\mathcal{L}_{\Theta}\right)\right|_{\mathcal{T}}=\omega_{\mathcal{T}}
$$

To specify the line bundle $\mathcal{L}_{\Theta}$ uniquely, one must choose a set of characteristics ${ }^{3} \Theta$ (more precisely, a quadratic refinement $\sigma_{\Theta}$ of the intersection form on $H^{3}(\mathcal{X}, \mathbb{Z})$ ) which determine the periodicity properties of the sections of $\mathcal{L}_{\Theta}$ under translations by $\Gamma$, i.e. under large gauge transformations $[28,34,35]$. By the Kodaira vanishing theorem, $\mathcal{L}_{\Theta}$ admits a unique holomorphic section $\vartheta_{\Theta}(\overline{\mathcal{N}}, C)$, which is proportional to the level $1 / 2$ Siegel theta series $\vartheta_{\text {Siegel }}\left[\begin{array}{l}\theta \\ \phi\end{array}\right]\left(\overline{\mathcal{N}}, \omega_{\Lambda}\right)$ of rank $b_{3}(\mathcal{X}) / 2$ with characteristics $\Theta=\left(\theta^{\Lambda}, \phi_{\Lambda}\right)$, where $\mathcal{N}$ is the (Weil) period matrix of $\mathcal{X}$ and $\omega_{\Lambda}$ are complex coordinates on the torus $\mathcal{T}$. Thus, the Gaussian flux partition function $\mathcal{Z}_{\mathrm{G}}^{(1)}$ for $k=1$ is given, up to a metric-dependent normalization factor, by the Siegel theta series $\vartheta_{\Theta}(\overline{\mathcal{N}}, C)$.

In general, for $k>1$ fivebranes wrapped on $\mathcal{X}$, the partition sum $\mathcal{Z}_{\mathrm{G}}^{(k)}$ over fluxes for the "diagonal" Gaussian self-dual three-form (related by supersymmetry to the scalar fields describing overall transverse fluctuations of the stack of fivebranes) is expected to be a holomorphic section of $\mathcal{L}_{\Theta}^{k}$. By the same vanishing theorem, $\mathcal{L}_{\Theta}^{k}$ admits $k^{b_{3}(\mathcal{X}) / 2}$ linearly independent holomorphic sections, given by level $k / 2$ generalizations of the Siegel theta series, and thus $\mathcal{Z}_{\mathrm{G}}^{(k)}$ is expected to be a linear combination of these theta series with coefficients depending on the metric on $\mathcal{X}$.

\subsection{NS5-brane partition function and non-Gaussian theta series}

Although it is a closely related object, the partition sum $\mathcal{Z}_{\mathrm{G}}^{(k)}$ of a Gaussian self-dual threeform reviewed above is not yet the fivebrane partition function $\mathcal{Z}_{\mathrm{NS} 5}^{(k)}$ relevant for instanton corrections to the hypermultiplet moduli space. There are several reasons for this:

i) Firstly, $\mathcal{Z}_{\mathrm{G}}^{(1)}$ only captures the topological degrees of freedom in the three-form $H$. However, for the purpose of computing NS5-instanton corrections to the HM moduli space metric, we require the partition function of the full $(2,0)$ supermultiplet on the brane world-volume. While the other fields in the multiplets do not couple to the $C$-field, they do contribute to the metric-dependent normalization factor.

ii) Secondly, one should include an insertion of $(-1)^{2 J_{3}}\left(2 J_{3}\right)^{2}$, where $J_{3}$ is a angular momentum operator on the fivebrane, in order to absorb fermionic zero-modes. Since the $H$-flux contributes to angular momentum, this may result in additional insertions in the Siegel theta series.

iii) Thirdly, the action for $H$ is Gaussian only in the limit where the flux $H$ is much smaller than the inverse string coupling $1 / g_{s}$. Thus, the Gaussian partition function $Z_{\mathrm{G}}^{(1)}$ is only valid at $g_{s}=0$, after factoring out a vanishingly small factor $e^{-S_{\mathrm{NS5}}}$ corresponding to the classical action $S_{\mathrm{NS} 5}=4 \pi e^{\phi}+\mathrm{i} \pi \sigma$ of the fivebrane. To compute

\footnotetext{
${ }^{3}$ The characteristics may in general depend on the metric on $\mathcal{X}$. They can in principle be computed in M-theory, see [33].
} 
the partition function at non-zero $g_{s}$, we require the full non-linear, kappa-symmetric action for the $(2,0)$ multiplet on $\mathcal{X}$, topologically twisted as a result of the insertion of $(-1)^{2 J_{3}}\left(2 J_{3}\right)^{2}$.

To address this last point in more detail, recall that two distinct kappa-symmetric descriptions of the $(2,0)$ multiplet have been developed in the literature: (a) The action constructed in [37-40], of Born-Infeld type, contains an auxiliary one-form (the gradient of a scalar field), whose equation of motion implies the self-duality condition; this action is well suited when $\mathcal{X}$ allows for globally well-defined oneforms, but seems inadequate for our purposes since for CY-threefolds $b_{1}(\mathcal{X})=0$. (b) In the second approach [41, 42], the action is polynomial and contains an auxiliary 5 -form, the elimination of which again leads to a Born-Infeld type action; the selfduality condition must however be imposed by hand (the action of $[41,42]$ is then more properly a pseudo-action). Using this approach, it may be possible to separate the self-dual and anti-self-dual parts of $H$ after a suitable Poisson resummation, generalizing the procedure in $[28,29,32,35]$, and then perform the topological twist.

iv) Finally, for $k>1$ fivebranes, the degrees of freedom on the fivebrane worldvolume are presumably no-longer field-theoretical, but should involve the full little string theory in 6 dimensions $[43,44]$. With our current understanding of this theory, there seems to be no way of fixing the linear combinations of level $k / 2$ theta series entering in $\mathcal{Z}_{\mathrm{G}}^{(k)}$ from first principles.

While all the reasons above show that the Gaussian theta series $\mathcal{Z}_{\mathrm{G}}^{(k)}$ and the fivebrane partition function $\mathcal{Z}_{\mathrm{NS} 5}^{(k)}$ governing instanton corrections to the HM moduli space are distinct functions of the metric and of the $C$-field, we nevertheless expect that both should transform in the same way under large gauge transformations of the $C$-field. Indeed, the latter depends only on the Wess-Zumino-type coupling between the $C$-field and the $H$-flux, which is independent of the detailed dynamics on the fivebrane world-volume. Thus we expect that $\mathcal{Z}_{\mathrm{G}}^{(k)}$ and $\mathcal{Z}_{\mathrm{NS} 5}^{(k)}$ are both sections of the same circle bundle $\mathcal{C}_{\Theta}=\mathcal{L}_{\Theta}^{\circ}$ over the intermediate Jacobian torus $\mathcal{T}$, where $\mathcal{L}_{\Theta}^{\circ}$ denotes the unit circle inside the line bundle $\mathcal{L}_{\Theta}$. In particular, $\mathcal{Z}_{\mathrm{NS} 5}^{(k)}$ should be given by a theta series with the same characteristics $\Theta$, albeit of non-Gaussian type. Unlike $\mathcal{Z}_{\mathrm{G}}^{(k)}$ however, $\mathcal{Z}_{\mathrm{NS5}}^{(k)}$ does not need to extend to a holomorphic section of $\mathcal{L}_{\Theta}$, and may have different metric dependence.

Supersymmetry further requires that $\mathcal{Z}_{\mathrm{NS5}}^{(k)}$ should be independent of the Kähler moduli (since the latter are part of the VM moduli space in type IIA), and that corrections induced from $\mathcal{Z}_{\mathrm{NS} 5}^{(k)}$ should preserve the quaternion-Kähler property of the HM metric. Thus, $\mathcal{Z}_{\mathrm{NS} 5}^{(k)}$ should (in a sense which we shall make precise below) lift to a holomorphic section on the twistor space $\mathcal{Z}_{\mathcal{M}}$ over $\mathcal{M}$. Using twistorial techniques as well as insights from S-duality and mirror symmetry, we shall construct the non-Gaussian theta series $\mathcal{Z}_{\mathrm{NS} 5}^{(k)}$, and show that its zero-coupling limit is a Gaussian theta series $\mathcal{Z}_{\mathrm{NS} \text {-G }}^{(k)}$. The latter is almost identical to the usual Gaussian flux partition function $\mathcal{Z}_{\mathrm{G}}^{(k)}$, but differs in its normalization factor, and in having an insertion of a power of $H$ in the sum over $H$-fluxes, cf. eq. (5.59) below. In particular, for $k=1$ the normalization factor of $\mathcal{Z}_{\mathrm{G}}^{(1)}$ can be expressed as a certain 
product of holomorphic Ray-Singer torsions, the same product which appears in the oneloop amplitude of the topological B-model [45]. ${ }^{4}$ This meshes nicely with the topology of the one-loop corrected HM moduli space, as we now discuss.

\subsection{Topology of the hypermultiplet moduli space and quantum corrections}

Irrespective of their microscopic origin, instanton corrections from $k$ fivebranes wrapped on $\mathcal{X}$ are characterized by their non-trivial dependence on the Neveu-Schwarz (NS) axion $\sigma$ through an overall phase factor $e^{-\mathrm{i} k \pi \sigma}$. Large gauge transformations of the $B$-field require that $\sigma$ is a compact coordinate with periodicity $\sigma \mapsto \sigma+2$ (in our conventions), consistent with the above exponential dependence of fivebrane instantons. Thus, $e^{\mathrm{i} \pi \sigma}, 0 \leq \sigma<2$ parametrizes the fiber of a circle bundle $\mathcal{C}$ over the intermediate Jacobian $\mathcal{J}_{c}(\mathcal{X})$. On the other hand, fivebrane instanton corrections to the HM metric are expected to be of the form

$$
\left.\delta \mathrm{d} s^{2}\right|_{\mathrm{NS} 5} \sim e^{-4 \pi|k| e^{\phi}-\pi \mathrm{i} k \sigma} \mathcal{Z}_{\mathrm{NS} 5-\mathrm{G}}^{(k)} .
$$

Indeed, as we show in section 3.2.1, such a coupling reproduces the classical fivebrane action computed from the 4D effective supergravity description in [49]. For the coupling (1.2) to be globally well-defined, the circle bundle $\mathcal{C}$ should be isomorphic to the circle bundle $\mathcal{C}_{\mathrm{NS} 5}$ where the fivebrane partition function $\mathcal{Z}_{\mathrm{NS} 5}^{(1)}$ is valued.

On the other hand, the perturbative moduli space metric (3.1) exhibits a one-loop correction to the kinetic term (3.2) of the NS-axion proportional to the Euler number $\chi(\mathcal{X})$ of the threefold $\mathcal{X}$. As we explain in section 3 , the origin of this correction can be traced to the familiar $B \wedge I_{8}$ topological coupling in 10 dimensions. This fact provides an alternative derivation of the one-loop correction to the HM metric, related by supersymmetry to the computation in $[13,15]$. In particular, the form of the axion kinetic term implies that the curvature of the connection on the circle bundle $\mathcal{C}$ is given by

$$
\mathrm{d}\left(\frac{D \sigma}{2}\right)=\omega_{\mathcal{T}}+\frac{\chi(\mathcal{X})}{24} \omega_{\mathcal{S K}}
$$

where $\omega_{\mathcal{T}}$ and $\omega_{\mathcal{S K}}$ are the Kähler forms on $\mathcal{T}$ and $\mathcal{M}_{c}$. The first term in (1.3) has support on the intermediate Jacobian torus $\mathcal{T}$. It reflects the fact that translations along the torus fiber $\mathcal{T}$ of $\mathcal{J}_{c}(\mathcal{X})$ (i.e. large gauge transformations of the $C$-field) commute only up to a translation along the circle fiber of $\mathcal{C}$ (i.e. a large gauge transformation of the $B$-field). As a result, large gauge transformations obey a Heisenberg group law, see eq. (3.11). Comparing (1.1) and (1.3), we see that $c_{1}(\mathcal{C})-c_{1}\left(\mathcal{C}_{\mathrm{NS} 5}\right)$ has no support on $\mathcal{T}$. Thus, the coupling (1.2) is indeed invariant under large gauge transformations, provided $e^{\mathrm{i} \pi \sigma}$ transforms in the same way as $\mathcal{Z}_{\mathrm{NS} \text {-G }}^{(1)}$, i.e. as a section of $\mathcal{L}_{\Theta}$.

The second term in (1.3), on the other hand, has support on the complex structure moduli space $\mathcal{M}_{c}(\mathcal{X})$, and implies that the NS-axion picks up anomalous variations under monodromies in $\mathcal{M}_{c}$. At this point it is worth pointing out that the $B \wedge I_{8}$ topological

\footnotetext{
${ }^{4}$ This connection to the topological B-model is not surprising in view of the fact that the BPS sector of the world-volume dynamics of a single M5-brane is governed by a deformation of Kodaira-Spencer theory [46-48]. It is also generally expected from mirror symmetry and S-duality, as we discuss in section 1.5.
} 
coupling, which is responsible for the second term in (1.3), is also responsible for an anomaly in the phase of the fivebrane partition function [28]. Indeed, the two effects are related by the anomaly inflow mechanism [50,51], which ultimately ensures that the fivebrane instanton coupling (1.2) is well-defined.

Now, the second term in (1.3), is equal to $\chi(\mathcal{X}) / 24$ times the curvature of the Hodge line bundle $\mathcal{L}$ over $\mathcal{M}_{c}(\mathcal{X})$, in which the holomorphic three-form $\Omega_{3,0}$ on $\mathcal{X}$ is valued. This indicates that the restriction of the circle bundle $\mathcal{C}$ to $\mathcal{M}_{c}(\mathcal{X})$ is $\mathcal{L}^{\chi(\mathcal{X}) / 24}$. Unless $\chi(\mathcal{X})$ is a multiple of 24 however, the definition of $\mathcal{L}^{\chi(\mathcal{X}) / 24}$ requires additional data, namely a homomorphism $M \mapsto e^{2 \pi \mathrm{i} \kappa(M)}$ from the monodromy group to the group of 24:th roots of unity - put differently, $e^{\mathrm{i} \pi \sigma}$ is valued in a twisted circle bundle. ${ }^{5}$ As a result, $\sigma$ transforms under monodromies according to

$$
\sigma \mapsto \sigma+\frac{\chi(\mathcal{X})}{24 \pi} \operatorname{Im} f_{M}+2 \kappa(M),
$$

where $f_{M}$ is a local holomorphic function on $\mathcal{M}_{c}(\mathcal{X})$ which determines the rescaling of the holomorphic three-form under monodromy, $\Omega_{3,0} \mapsto e^{f_{M}} \Omega_{3,0}$. (Here one must make a choice of branch of the logarithm in defining $f_{M}$, correlated with the choice of branch in defining $\kappa(M)$.) In order for the product (1.2) to be well-defined, the fivebrane partition function $\mathcal{Z}_{\text {NS5-G }}^{(1)}$ should transform in the opposite fashion. Since, as mentioned at the end of the previous subsection, $\mathcal{Z}_{\mathrm{NS5-G}}^{(k)}$ is proportional to the holomorphic part $e^{f_{1}}$ of the B-model one-loop amplitude, this entails that $e^{2 \pi \mathrm{i} \kappa(M)}$ also governs the modular properties of $e^{f_{1}}$ under monodromies in $\mathcal{M}_{c}$.

\subsection{Quantum mirror symmetry, S-duality and $(p, k)$ fivebranes}

The NS5-brane instanton corrections to the HM metric may also be obtained using mirror symmetry $^{6}$ and S-duality. Indeed, according to the quantum mirror symmetry conjecture [3] (see $[22,23,54]$ for recent discussions), the same moduli space $\mathcal{M}$ must arise as the HM moduli space of type IIB string theory compactified on the mirror threefold $\hat{\mathcal{X}}$. At the perturbative level, this amounts to the classical mirror symmetry statement $\mathcal{M}_{c}(\mathcal{X})=\mathcal{M}_{K}(\hat{\mathcal{X}})$ between the complex structure moduli space of $\mathcal{X}$ and the complexified Kähler moduli space of $\hat{\mathcal{X}}$, the one-loop correction being controlled by the same invariant $\chi(\mathcal{X})=-\chi(\hat{\mathcal{X}})$ on both sides. At the non-perturbative level, the identity between the two HM moduli spaces requires a matching between the D-instanton effects on the type IIA side, corresponding to D2-branes wrapped on special Lagrangian submanifolds of $\mathcal{X}$, and D5-D3-D1-D(-1)-instantons on the type IIB side, corresponding to stable coherent sheaves on $\hat{\mathcal{X}}$. In section 4.1 , we explain how the fractional charges predicted by the K-theory description of D5-D3-D1-D(-1)-instantons are consistent with the fact that D2brane charges are classified ${ }^{7}$ by $H_{3}(\mathcal{X}, \mathbb{Z})$. The resolution of this apparent paradox hinges

\footnotetext{
${ }^{5}$ We are grateful to G. Moore, A. Neitzke and D. Zagier for discussions which have helped shaping this point of view.

${ }^{6}$ For rigid Calabi-Yau threefolds, mirror symmetry is not available. NS5-instanton effects in this case have been recently addressed in $[52,53]$ by postulating automorphic symmetries.

${ }^{7}$ It would be interesting to formulate mirror symmetry directly at the level of the K-theory groups $K(\hat{\mathcal{X}})$ and $K^{1}(\mathcal{X})$, but we shall not attempt to do so here.
} 
on the quadratic ambiguity $A_{\Lambda \Sigma}$ of the prepotential (4.4) in the large volume limit, and in fact allows to derive constraints on the fractional part of the matrix $A_{\Lambda \Sigma}$, some of which had been observed in the early days of classical mirror symmetry [55-58]. Incorporating these effects leads to novel corrections to the "Ramond-Ramond mirror map" and S-duality action derived in $[14,18,20]$, which we exhibit in eqs. (4.18) and (4.31).

Assuming that these D-instanton corrections do match on both sides of the mirror map, quantum mirror symmetry further requires that fivebrane corrections on the type IIA (or M-theory) side match NS5-brane corrections, or more generally $(p, k) 5$-brane corrections, on the type IIB side. The latter are related by type IIB S-duality ${ }^{8}$ to D5-brane instantons, governed by the generalized DT invariants $\Omega(\gamma)$. Thus, by combining S-duality and mirror symmetry, one expects that the type IIA NS5-instanton contributions are expressible in terms of the topological B-model amplitude on $\mathcal{X}[32,59]$ for $k=1$, and more generally in terms of the generalized DT invariants of $\mathcal{X}$.

To implement these ideas, we start from holomorphic sections of $H^{1}\left(\mathcal{Z}_{\mathcal{M}}, \mathcal{O}(2)\right)$ describing D5-D3-D1-D(-1) instanton configurations, with charges $\gamma=\left(p^{0}, p^{a}, q_{a}, q_{0}\right)$, and sum over their images under S-duality. This twistor construction ensures that all instanton corrections respect the quaternion-Kähler property of the metric, as required by supersymmetry. The resulting (local) holomorphic functions encode corrections to the contact structure from $(p, k)$-brane instantons with $\operatorname{gcd}(p, k)=p^{0}$, bound to D3-D1-D(-1) instantons, and take the following schematic form

$$
H_{\mathrm{NS} 5}^{(k)}(\xi, \tilde{\xi}, \tilde{\alpha}) \sim e^{-\mathrm{i} \pi k \tilde{\alpha}} H_{\mathrm{NS} 5}^{(k)}(\xi, \tilde{\xi})
$$

where the variables $(\xi, \tilde{\xi}, \tilde{\alpha})$ are complex Darboux coordinates on $\mathcal{Z}_{\mathcal{M}}$. These functions form an invariant set under the Heisenberg action (3.15) and under monodromies around the point of infinite volume in $\mathcal{M}_{K}(\hat{\mathcal{X}})$, up to subtle phases which we compute in appendix A. These phases indicate some tension between S-duality, Heisenberg and monodromy invariance, and suggest that it may be necessary to relax some of our assumptions about the way these symmetries are realized. In the absence of a satisfactory resolution and since, by and large, our results seem to support our general assumptions, we dauntlessly forge ahead.

Ignoring these subtle phases then, the holomorphic functions (1.5) can be combined into a formal Poincaré series, and rewritten as a non-Gaussian theta series with kernel given in (5.37). For $k=1$, the summand of the theta series can be recognized, using the DT/GW relation between (ordinary, rank one) Donaldson-Thomas invariants and Gromov-Witten invariants [60, 61], as the topological amplitude of the A-model on $\hat{\mathcal{X}}$ (see eq. (5.42) below). Thus, the partition function of a single type IIB NS5-brane is given by a theta series built on the A-model topological string amplitude. Using mirror symmetry, the partition function of a single type IIA NS5-brane is then also a theta series whose wave function coincides with that of the B-model on $\mathcal{X}$. By similar arguments, the partition function of type IIB $(p, k) 5$-branes is governed by $\operatorname{rank} r=\operatorname{gcd}(p, k)$ Donaldson-Thomas invariants on $\hat{\mathcal{X}}$.

\footnotetext{
${ }^{8}$ As in earlier investigations $[18,20,21]$, we shall work under the assumption that the full $\operatorname{SL}(2, \mathbb{Z})$ S-duality of ten-dimensional type IIB string theory stays valid in vacua with $\mathcal{N}=2$ supersymmetry.
} 


\subsection{Fivebrane partition function and twistors}

The object $H_{\mathrm{NS} 5}^{(k)}(\xi, \tilde{\xi})$ in (1.5) is formally a holomorphic section of $H^{1}\left(\mathcal{Z}_{\mathcal{M}}, \mathcal{O}(2)\right)$, and provides, by a standard (though cumbersome) procedure described e.g. in [12], an infinitesimal deformation of the metric tensor on $\mathcal{M}$ consistent with the quaternion-Kähler property. By construction, $H_{\mathrm{NS} 5}^{(k)}(\xi, \tilde{\xi})$ and the phase $e^{-\mathrm{i} \pi k \tilde{\alpha}}$ are separately invariant under rescaling of the holomorphic three-form $\Omega_{3,0}$ (up to subtleties in the definition of $\mathcal{L}^{\chi(\mathcal{X}) / 24}$ mentioned at the end of section 1.4). Moreover, similarly as in (1.2), the two factors in (1.5) transform oppositely under translations along the torus $\mathcal{T}$. Hence the fivebrane correction (1.5) to the complex contact structure on $\mathcal{Z}$ is well-defined under both large gauge transformations and monodromies (up to subtle phases that we ignore).

While this gives a (partial) answer to the question of main interest in this paper, namely to compute fivebrane instanton corrections to the metric on $\mathcal{M}$, one may ask a different though related question: is it possible to construct a scalar-valued function on $\mathcal{M}$ which would generalize the Gaussian fivebrane partition function $\mathcal{Z}_{\mathrm{G}}^{(k)}$ at finite coupling? A possible answer to this question is obtained by viewing (1.5) instead as a formal holomorphic section of $H^{1}\left(\mathcal{Z}_{\mathcal{M}}, \mathcal{O}(-2)\right)$ (barring global issues) and applying the standard Penrose transform, which relates sections of $H^{1}\left(\mathcal{Z}_{\mathcal{M}}, \mathcal{O}(-2)\right)$ to functions on $\mathcal{M}$ satisfying a certain set of second-order partial differential equations (see e.g. [10]). While this procedure is not rigorous in general, it has been made rigorous in the case of fourdimensional quaternion-Kähler manifolds (see [62]), and can also serve as a warm-up for the more intensive computations involved in extracting the explicit metric on $\mathcal{M}$. In either case, the answer involves contour integrals over the $\mathbb{C} P^{1}$ fiber of the twistor space $\mathcal{Z}$, and reduces to the same saddle points in certain classical limits.

Performing this computation in the large volume limit, we indeed find that the Penrose transform of (1.5) reduces to a non-Gaussian theta series, whose summand displays the expected non-linear action for $(p, k) 5$-branes. Moreover, in the zero coupling limit, this reduces to a Gaussian partition function of the type discussed in section 1.2, with a normalization factor given by the same product of holomorphic Ray-Singer torsions which appears in the one-loop amplitude of the topological B-model on $\mathcal{X}$, and an additional fluxdependent insertion in the sum. As a side-product, we also find a twistorial interpretation for the "auxiliary parameter" $t$ introduced by [32] to relate the Griffiths and Weil complex structures on the intermediate Jacobian of $\mathcal{X}$.

\subsection{Outline}

The remainder of this article is organized as follows. • In section 2, we review the construction of the partition function of a Gaussian self-dual 3-form by holomorphic factorization, and we recall some useful facts about topological strings. - In section 3, we discuss the topology of the hypermultiplet moduli space, using the qualitative form of D-instanton and NS5-instanton corrections to identify the periodicities of the RR and NS axions. - Then, in section 4, we describe the corresponding type IIB picture and, using mirror symmetry and S-duality, we find the classical action for general fivebrane instantons, and show that it reduces to the Gaussian flux action in the weak coupling limit. • In section 5, we review 
the twistorial description of the perturbative and D-instanton corrected HM moduli space, and obtain a candidate section of $H^{1}\left(\mathcal{Z}_{\mathcal{M}}, \mathcal{O}(2)\right)$ which should govern deformations of the complex contact structure on twistor space $\mathcal{Z}_{\mathcal{M}}$ induced by $(p, k)$-fivebranes. The partition function $\mathcal{Z}_{\text {NS5 }}^{(k)}$ of $k$ NS5-branes, including non-linear effects, follows by Penrose transform from this holomorphic section. - We conclude in section 6 with a summary and discussion. - The transformation properties of the candidate fivebrane transition functions $H_{k, p, \hat{\gamma}}$ under various actions are discussed in appendix A, and a directory of notations is provided in appendix B. Note in particular the pervasive use of the notation $\mathbf{E}(z)=\exp [2 \pi \mathrm{i} z]$.

\section{Flux partition functions and topological wave functions}

In this section we begin by revisiting previous constructions of the fivebrane partition function, originally spelled out in [28] and further discussed in [30, 32-35]. We also review some relevant background material on topological strings, which will play an important role in section 5 .

- In section 2.1, we set up the problem and introduce our conventions for the moduli space of metrics and RR $C$-fields on $\mathcal{X}$. - In section 2.2, we obtain, up to an overall metric-dependent factor, the partition function $\mathcal{Z}_{\mathrm{G}}^{(k)}$ for a self-dual 3-form, by holomorphic factorization of the partition function of a non-chiral Gaussian harmonic three-form. In this construction, a key role is played by a certain $\mathbb{Z}_{2}$-valued function $\sigma_{\Theta}$ (a quadratic refinement of the intersection form on $H^{3}(\mathcal{X}, \mathbb{Z})$ modulo 2). As we discuss in section 2.3, the quadratic refinement $\sigma_{\Theta}$ ensures that $\mathcal{Z}_{\mathrm{G}}^{(k)}$ is valued in a single line bundle $\mathcal{L}_{\Theta}^{k}$ with characteristics $\Theta \in(\mathbb{Z} / 2 \mathbb{Z})^{b_{3}}$. • In section 2.4 we discuss non-Gaussian generalizations of this partition function and their interpretation in terms of certain wave functions.

Section 2.5 is then devoted to a discussion of various aspects of the topological A- and B-model, with particular emphasis on the wave function properties of their respective partition functions. In particular, we recall some useful relations between topological wave functions and the partition functions of Donaldson-Thomas and Gromov-Witten invariants.

Our exposition in sections 2.2 and 2.3 follows closely [28, 35] but is specifically geared towards the partition function of the NS5-brane on a Calabi-Yau threefold. As was already stressed in section $1, \mathcal{Z}_{\mathrm{G}}^{(k)}$ is not the partition function of the NS5-brane, but only an approximation thereof. In order to construct $\mathcal{Z}_{\mathrm{G}}^{(k)}$ through holomorphic factorization, one is forced to make some simplifying assumptions, which we find in section 5 are in fact not valid to accurately describe the NS5-brane. Nevertheless, we find it useful and illuminating to go through the derivation of $\mathcal{Z}_{\mathrm{G}}^{(k)}$ in some detail in our conventions in order to facilitate the comparison with the correct NS5-partition function constructed in section 5 using twistor techniques. Moreover, $\mathcal{Z}_{\mathrm{G}}^{(k)}$ does capture many of the key topological features which are expected of the NS5-brane partition function.

\subsection{Setting the stage}

Let us consider type IIA string theory compactified on a Calabi-Yau threefold $\mathcal{X}$, with a stack of $k$ Euclidean NS5-branes whose world-volume $W$ consists of $\mathcal{X}$ itself. For $k=1$, the world-volume $W$ supports 5 scalar fields (describing transverse fluctuations of $W$ in 
$\mathbb{R}^{10} \times S^{1}$, where $S^{1}$ is the M-theory circle), two symplectic Majorana-Weyl fermions and a 2-form potential $\mathcal{B}$, whose 3 -form field strength $H=\mathrm{d} \mathcal{B}$ must be imaginary self-dual [5],

$$
{ }_{W} H=\mathrm{i} H .
$$

The world-volume $W$ is a magnetic source for the NS 2-form potential $B$ propagating in the 10-dimensional bulk of space-time, while the flux $H$ is an electric source for the RR 3 -form potential $C$, mimicking D2-branes bound to the NS5-brane.

For $k>1$ fivebranes wrapping $\mathcal{X}$, the world-volume dynamics is poorly understood, but presumably splits into "twisted sectors" labeled by pairs of integers $(p, q)$ such that $p q=k$ [35], where the $k$ fivebranes on $\mathcal{X}$ recombine into $q$ fivebranes wrapping $p$ copies of $\mathcal{X}$. The dynamics in the $(p, q)$ twisted sector should be described by $q^{3}$ interacting self-dual two-forms $\mathcal{B}_{i j k}, i, j, k=1 \ldots q$, such that the $q$ two-forms $\mathcal{B}_{i i i}$ stay massless on the Coulomb branch. We shall focus on the Abelian 2-form $\mathcal{B}=\sum_{i=1 \ldots q} \mathcal{B}_{i i i}$ describing the center of mass degrees of freedom. Moreover, we restrict to a regime where $g_{s}|H| \ll 1$, such that the flux $H$ contributes quadratically to the fivebrane action.

Our goal in this section is to revisit the construction of the Gaussian topological partition sum $\mathcal{Z}_{\mathrm{G}}^{(k)}$ for the field $H$ together with its supersymmetric partners, as a function of the moduli of the $\mathrm{CY}$ threefold $\mathcal{X}$ and the periods of the background $C$-field on $\mathcal{X}$. By topological partition sum, we mean the partition sum weighted by $(-1)^{2 J_{3}}\left(2 J_{3}\right)^{2}$, which is relevant for two-derivative couplings in the low energy effective action. As already mentioned in section 1.3 , the assumption that $g_{s}|H| \ll 1$ eventually breaks down for large values of the flux, and the Gaussian action should be replaced by its non-linear, Born-Infeld type completion [37-41] in order to obtain the physical NS5-brane partition function. We shall return to this issue in section 5.5.

As will become apparent shortly, $\mathcal{Z}_{\mathrm{G}}^{(k)}$ is independent of the Kähler structure of $\mathcal{X}$, and depends only on its complex structure. The moduli space $\mathcal{M}_{c}(\mathcal{X})$ of complex structures can be parametrized as usual by the complex periods

$$
X^{\Lambda}=\int_{\mathcal{A}^{\Lambda}} \Omega_{3,0}, \quad F_{\Lambda}=\int_{\mathcal{B}_{\Lambda}} \Omega_{3,0}
$$

of the holomorphic 3 -form ${ }^{9} \Omega$ along a symplectic basis $\left(\mathcal{A}^{\Lambda}, \mathcal{B}_{\Lambda}\right), \Lambda=0, \ldots, h_{2,1}(\mathcal{X})$, of $H_{3}(\mathcal{X}, \mathbb{Z})$ modulo holomorphic rescalings $\Omega_{3,0} \mapsto e^{f} \Omega_{3,0}$. We shall denote $\Omega \equiv\left(X^{\Lambda}, F_{\Lambda}\right)^{T}$. The vector $\Omega$ thus takes values in an homogeneous complex Lagrangian cone $\mathcal{C}_{\mathcal{X}}$, generated locally by a prepotential $F\left(X^{\Lambda}\right)$ such that $F_{\Lambda}=\partial_{X^{\Lambda}} F(X)$. The complex structure moduli space $\mathcal{M}_{c}(\mathcal{X})$ is the quotient $\mathcal{C}_{X} / \mathbb{C}^{\times}$by homogeneous rescalings, which we parametrize by complex coordinates $z^{a}$. Conversely, the cone $\mathcal{C}_{X}$ provides a canonical complex line bundle over $\mathcal{M}_{c}(\mathcal{X})$, known as the Hodge line bundle, which we denote by $\mathcal{L}$. Recall that $\mathcal{M}_{c}(\mathcal{X})$ carries a special Kähler metric, with Kähler potential

$$
\mathcal{K}=-\log \left[\mathrm{i}\left(\bar{X}^{\Lambda} F_{\Lambda}-X^{\Lambda} \bar{F}_{\Lambda}\right)\right]
$$

\footnotetext{
${ }^{9}$ We trust that the reader will not confuse the holomorphic 3-form $\Omega$ with the generalized DonaldsonThomas invariant $\Omega(\gamma)$. The latter will always come with an argument.
} 
and Kähler class $\omega_{\mathcal{S K}}=-\frac{1}{2 \pi} \mathrm{d} \mathcal{A}_{K}$, where

$$
\mathcal{A}_{K}=\frac{\mathrm{i}}{2}\left(\mathcal{K}_{a} \mathrm{~d} z^{a}-\mathcal{K}_{\bar{a}} \mathrm{~d} \bar{z}^{\bar{a}}\right)
$$

is the Kähler connection. ${ }^{10}$ Under a monodromy $M$ in $\mathcal{M}_{c}(\mathcal{X})$, the symplectic basis $\left(\mathcal{A}^{\Lambda}, \mathcal{B}_{\Lambda}\right)$ transforms by an integer-valued symplectic rotation $\rho(M)=\left(\begin{array}{ll}\mathcal{D} & \mathcal{C} \\ \mathcal{B} & \mathcal{A}\end{array}\right) \in$ $\operatorname{Sp}\left(b_{3}(\mathcal{X}), \mathbb{Z}\right)$ with $b_{3}(\mathcal{X})=2 h_{2,1}+2$. At the same time $\Omega_{3,0} \mapsto e^{f_{M}} \Omega_{3,0}$ is generically rescaled, so the period vector $\Omega$ transforms into $e^{f_{M}} \rho(M) \Omega$. In the process, $\mathcal{K}$ and $\mathcal{A}$ change respectively by Kähler transformations and gauge transformations of the form

$$
\mathcal{K} \longmapsto \mathcal{K}-f_{M}-\bar{f}_{M}, \quad \mathcal{A}_{K} \longmapsto \mathcal{A}_{K}+\mathrm{d} \operatorname{Im}\left(f_{M}\right) .
$$

A topologically trivial real $C$-field on $\mathcal{X}$ can be parametrized by its real periods

$$
\zeta^{\Lambda}=\int_{\mathcal{A}^{\Lambda}} C, \quad \tilde{\zeta}_{\Lambda}=\int_{\mathcal{B}_{\Lambda}} C .
$$

Again, we shall abuse notation and write $C$ for the vector $\left(\zeta^{\Lambda}, \tilde{\zeta}_{\Lambda}\right)^{T}$. By charge quantization, the entries of $C$ are periodic with unit periods, and parametrize a point on the $b_{3}(\mathcal{X})$-dimensional real torus

$$
\mathcal{T}=H^{3}(\mathcal{X}, \mathbb{R}) / H^{3}(\mathcal{X}, \mathbb{Z})
$$

equipped with the canonical symplectic form ${ }^{11}$

$$
\omega_{\mathcal{T}}=-\int_{\mathcal{X}} \mathrm{d} C \wedge \mathrm{d} C=\mathrm{d} \tilde{\zeta}_{\Lambda} \wedge \mathrm{d} \zeta^{\Lambda}
$$

The torus $\mathcal{T}$ is fibered over the complex structure moduli space $\mathcal{M}_{c}(\mathcal{X})$, such that $\left(\zeta^{\Lambda}, \tilde{\zeta}_{\Lambda}\right)$ transforms by the symplectic rotation $\rho(M)$ under a monodromy $M$. We shall refer to the total space of this fibration,

$$
\begin{aligned}
H^{3}(\mathcal{X}, \mathbb{R}) / H^{3}(\mathcal{X}, \mathbb{Z}) \rightarrow & \mathcal{J}_{c}(\mathcal{X}) \\
& \downarrow \\
& \mathcal{M}_{c}(\mathcal{X})
\end{aligned}
$$

as the intermediate Jacobian $\mathcal{J}_{c}(\mathcal{X})$. The Hodge star $\star \mathcal{X}$ on $H^{3}(\mathcal{X}, \mathbb{R})$ endows the fiber $\mathcal{T}$ with a complex structure and a positive-definite Kähler metric

$$
\mathrm{d} s_{\mathcal{T}}^{2}=\frac{1}{2} \int_{\mathcal{X}} \mathrm{d} C \wedge \star \mathcal{X} \mathrm{d} C=-\frac{1}{2} \mathrm{~d} \omega_{\Lambda} \operatorname{Im} \mathcal{N}^{\Lambda \Sigma} \mathrm{d} \bar{\omega}_{\Sigma}
$$

where the complex coordinates $\omega_{\Lambda}$ are given by

$$
\omega_{\Lambda}=\tilde{\zeta}_{\Lambda}-\overline{\mathcal{N}}_{\Lambda \Sigma} \zeta^{\Sigma}, \quad \bar{\omega}_{\Lambda}=\tilde{\zeta}_{\Lambda}-\mathcal{N}_{\Lambda \Sigma} \zeta^{\Sigma} .
$$

\footnotetext{
${ }^{10}$ Note that our definition of $\mathcal{A}_{K}$ differs by a factor of 2 from that used in $[12,17,18]$.

${ }^{11}$ In this equation and (2.10) below, $\mathrm{d} C$ denotes the variation of $C$, not its exterior derivative on $\mathcal{X}$.
} 
The Kähler class of the metric on $\mathcal{T}$ is the symplectic form $\omega_{\mathcal{T}}$ in (2.8) above. Equipped with the complex structure just defined, which we refer to as the Weil complex structure, $\mathcal{T}$ is a principally polarized Abelian variety.

The Weil period matrix $\mathcal{N}_{\Lambda \Sigma}$ is related to the Griffiths period matrix $\tau_{\Lambda \Sigma}=\partial_{\Lambda} \partial_{\Sigma} F(X)$ via the usual special geometry relation

$$
\mathcal{N}_{\Lambda \Lambda^{\prime}}=\bar{\tau}_{\Lambda \Lambda^{\prime}}+2 \mathrm{i} \frac{[\operatorname{Im} \tau \cdot X]_{\Lambda}[\operatorname{Im} \tau \cdot X]_{\Lambda^{\prime}}}{X^{\Sigma} \operatorname{Im} \tau_{\Sigma \Sigma^{\prime}} X^{\Sigma^{\prime}}}
$$

Recall that $\operatorname{Im} \mathcal{N}_{\Lambda \Sigma}$ and its inverse $\operatorname{Im} \mathcal{N}^{\Lambda \Sigma}$ are negative definite symmetric matrices, while $\operatorname{Im} \tau_{\Lambda \Sigma}$ and its inverse $\operatorname{Im} \tau^{\Lambda \Sigma}$ have signature $\left(1, b_{3}(\mathcal{X})-1\right)$. Under monodromies in $\mathcal{M}_{c}(\mathcal{X})$, both $\mathcal{N}_{\Lambda \Lambda^{\prime}}$ and $\tau_{\Lambda \Lambda^{\prime}}$ transform by fractional linear transformations

$$
\mathcal{N} \mapsto(\mathcal{A N}+\mathcal{B})(\mathcal{C N}+\mathcal{D})^{-1}, \quad \tau \mapsto(\mathcal{A} \tau+\mathcal{B})(\mathcal{C} \tau+\mathcal{D})^{-1}
$$

However, unlike the Griffiths period matrix $\tau_{\Lambda \Sigma}$, the Weil period matrix $\mathcal{N}_{\Lambda \Sigma}$ does not vary holomorphically over $\mathcal{M}_{c}(\mathcal{X})$.

As is well-known, the self-duality constraint on $H$ leads to several complications in defining its partition function. A self-dual flux $H$ can only couple to the anti-self-dual part of the background 3 -form $C$, so one would naively expect $\mathcal{Z}_{\mathrm{G}}^{(k)}$ to be a holomorphic function on $\mathcal{T}$, independent of $\bar{\omega}_{\Lambda}$. Instead, as emphasized in [28] and reviewed in detail below, $\mathcal{Z}_{\mathrm{G}}^{(k)}$ is more properly viewed as a holomorphic section of a certain line bundle over the torus $\mathcal{T}$. As in the case of two-dimensional chiral bosons [63, 64], it is convenient to obtain the partition function of a chiral 2-form by factorizing the partition function of a non-chiral two-form.

\subsection{Factorizing the non-chiral partition function}

To construct the partition function we first ignore the self-duality condition on the self-dual 3 -form, and restrict to the limit of small string coupling. Then the action for the 3 -form flux on the world-volume $p \mathcal{X}$ of $q$ fivebranes is given by $[28,35]$

$$
S(H, C)=p\left[\frac{\pi}{q} \int_{\mathcal{X}}(H-q C) \wedge \star(H-q C)-\mathrm{i} \pi \int_{\mathcal{X}} C \wedge H\right] .
$$

The overall factor of $p$ reflects the wrapping number of each fivebrane on $\mathcal{X}$, while the $1 / q$ factor is the familiar fractionization effect, coming from expanding $\sqrt{q^{2} / g_{s}^{4}+H^{2} / g_{s}^{2}}-|q| / g_{s}^{2}$ at small coupling. The factor of $q$ in $H-q C$ arises by summing $H_{i i i}-C$ over $i=1 \ldots q$.

Flux quantization requires that $H \in H^{3}(\mathcal{X}, \mathbb{Z})$, i.e. that the periods

$$
n^{\Lambda}=\int_{\mathcal{A}^{\Lambda}} H, \quad m_{\Lambda}=\int_{\mathcal{B}_{\Lambda}} H
$$

be integer valued. Using (2.10), the classical action (2.14) then reads

$$
S(\mathcal{N}, H, C)=-\frac{\pi p}{2 q}\left(\tilde{m}_{\Lambda}-\mathcal{N}_{\Lambda \Lambda^{\prime}} \tilde{n}^{\Lambda^{\prime}}\right) \operatorname{Im} \mathcal{N}^{\Lambda \Sigma}\left(\tilde{m}_{\Sigma}-\overline{\mathcal{N}}_{\Sigma \Sigma^{\prime}} \tilde{n}^{\Sigma^{\prime}}\right)-\mathrm{i} \pi p\left(m_{\Lambda} \zeta^{\Lambda}-n^{\Lambda} \tilde{\zeta}_{\Lambda}\right),
$$


where we defined $\tilde{m}_{\Lambda}=m_{\Lambda}-q \tilde{\zeta}_{\Lambda}, \tilde{n}^{\Lambda}=n^{\Lambda}-q \zeta^{\Lambda}$. We also indicated the dependence on the period matrix $\mathcal{N}$. As advertised above, (2.16) is independent of the Kähler moduli of $\mathcal{X}$. In terms of the self-dual and anti-self-dual components of the flux $H$,

$$
R_{\Lambda}=m_{\Lambda}-\overline{\mathcal{N}}_{\Lambda \Sigma} n^{\Sigma}, \quad \bar{R}_{\Lambda}=m_{\Lambda}-\mathcal{N}_{\Lambda \Sigma} n^{\Sigma},
$$

and of the $C$-field, eq. (2.11), this is equivalently rewritten (in agreement with [35], eq. 2.4) as

$$
S(\mathcal{N}, H, C)=-\operatorname{Im} \mathcal{N}^{\Lambda \Sigma}\left[\frac{\pi p}{2 q} R_{\Lambda} \bar{R}_{\Sigma}-\pi p \bar{\omega}_{\Lambda} R_{\Sigma}+\frac{\pi p q}{2} \omega_{\Lambda} \bar{\omega}_{\Sigma}\right] .
$$

Thus, the self-dual part $\omega_{\Lambda}$ of $C$ decouples, save for the last term in (2.18) which originates from the quadratic term $C \wedge \star_{\mathcal{X}} C$ in (2.14).

The partition function for a Gaussian 3-form flux in the twisted sector $(p, q)$, with $k=p q$, is now obtained as

$$
\mathcal{Z}_{\mathrm{G}}^{(p, q)}(\mathcal{N}, C)=\sum_{H \in H^{3}(\mathcal{X}, \mathbb{Z})} r(\mathcal{N}, H, C) e^{-S(\mathcal{N}, H, C)},
$$

where the prefactor $r(\mathcal{N}, H, C)$ corresponds to the fluctuation determinant around the harmonic flux configuration $H=n^{\Lambda} \alpha_{\Lambda}-m_{\Lambda} \beta^{\Lambda}$, where $\left(\alpha_{\Lambda}, \beta^{\Lambda}\right)$ is the symplectic basis on the lattice $\Gamma=H^{3}(\mathcal{X}, \mathbb{Z})$ dual ${ }^{12}$ to $\left(\mathcal{A}^{\Lambda}, \mathcal{B}_{\Lambda}\right)$. In view of our ignorance of the non-Abelian dynamics of $q$ fivebranes, we do not know how to evaluate $r(\mathcal{N}, H, C)$ from first principles. Nevertheless, it was argued in $[34,35]$ on topological grounds that $r(\mathcal{N}, H, C)$ must take the form

$$
r(\mathcal{N}, H, C)=|\mathcal{F}|^{2}[\sigma(H)]^{k},
$$

where the normalization factor $|\mathcal{F}|^{2}$ depends only on the metric on $\mathcal{X}$ (and possibly, on the string coupling), but not on $H$-flux nor on the background $C$-field. We shall see in section 5.5 that this assumption does not hold for the fivebrane instanton partition $\mathcal{Z}_{\text {NS5-G }}^{(k)}$ derived from twistor techniques, where the factor $|\mathcal{F}|^{2}$ appears to depend on $H$ and $C$, see (5.60) below. This dependence however is unessential for the topological properties that we wish to emphasize here, and we proceed in the remainder of this section under the assumption that $|\mathcal{F}|^{2}$ is independent of the flux $H$ and the background field $C$.

The second (essential) factor $\sigma(H)$ in $(2.20)$ is a "quadratic refinement of the intersection form" on the lattice $\Gamma$. This is defined as a homomorphism $\sigma: \Gamma \rightarrow \mathrm{U}(1)$, i.e. a phase assignment such that

$$
\sigma\left(H+H^{\prime}\right)=(-1)^{\left\langle H, H^{\prime}\right\rangle} \sigma(H) \sigma\left(H^{\prime}\right)
$$

where

$$
\left\langle H, H^{\prime}\right\rangle=m_{\Lambda} n^{\prime \Lambda}-m_{\Lambda}^{\prime} n^{\Lambda} .
$$

The most general solution of (2.21) is given by [35]

$$
\sigma_{\Theta}(H)=\mathbf{E}\left(-\frac{1}{2} m_{\Lambda} n^{\Lambda}+m_{\Lambda} \theta^{\Lambda}-n^{\Lambda} \phi_{\Lambda}\right),
$$

\footnotetext{
${ }^{12}$ Namely, $\int_{\mathcal{A}^{\Lambda}} \alpha_{\Sigma}=\delta_{\Sigma}^{\Lambda}, \int_{\mathcal{B}_{\Lambda}} \beta^{\Sigma}=-\delta_{\Lambda}^{\Sigma}, \int_{\mathcal{A}^{\Lambda}} \beta^{\Sigma}=\int_{\mathcal{B}^{\Lambda}} \alpha_{\Sigma}=0$.
} 
where $\Theta=\left(\theta^{\Lambda}, \phi_{\Lambda}\right)^{T}$ are independent of $H$ and defined modulo $\Gamma$. As we shall see below, $\Theta$ determines the characteristics of the theta series governing the chiral partition function. By analogy with the standard Jacobi theta series, $\Theta$ is sometimes referred to as a "generalized spin structure" on $\mathcal{X}$. $\Theta$ in general depends on the metric on $\mathcal{X}$ : under monodromies in $\mathcal{M}_{c}(\mathcal{X}), \Theta$ transforms as

$$
\left(\begin{array}{c}
\theta^{\Lambda} \\
\phi_{\Lambda}
\end{array}\right) \mapsto \rho(M) \cdot\left[\left(\begin{array}{c}
\theta^{\Lambda} \\
\phi_{\Lambda}
\end{array}\right)-\frac{1}{2}\left(\begin{array}{c}
\left(\mathcal{A}^{T} \mathcal{C}\right)_{d} \\
\left(\mathcal{D}^{T} \mathcal{B}\right)_{d}
\end{array}\right)\right],
$$

where $(A)_{d}$ denotes the diagonal of a matrix $A$ [65]. Since the $H$-flux transforms as $H \mapsto$ $\rho(M) \cdot H$, the quadratic refinement $\sigma_{\Theta}(H)$ is invariant. Moreover, while it is consistent to assume that $\sigma_{\Theta}^{2}(H)=1,2 \Theta \in \Gamma$ for the partition of a self-dual three-form [28, 35], in general, this is not so for the full fivebrane partition function, where $\sigma_{\Theta}(H)$ may be $\mathrm{U}(1)$-valued. In this subsection, we nevertheless assume that $\sigma_{\Theta}^{2}(H)=1$, which simplifies the holomorphic factorization procedure. We shall relax this assumption in section 2.3 onwards.

In order to decouple the self-dual and anti-self dual parts of $H$, we now split the lattice $\Gamma=H^{3}(\mathcal{X}, \mathbb{Z})$ into the sum of two Lagrangian lattices $\Gamma_{e} \oplus \Gamma_{m}$ (where ' $e$ ' and ' $m$ ' stand for 'electric' and 'magnetic'), given by

$$
\Gamma_{e}=\sum_{\Lambda} \mathbb{Z} \beta^{\Lambda}, \quad \Gamma_{m}=\sum_{\Lambda} \mathbb{Z} \alpha_{\Lambda}
$$

and perform a Poisson resummation on the integers $m_{\Lambda} \in \Gamma_{e}$. Denoting by $r^{\Lambda} \in \Gamma_{e}^{*}=\Gamma_{m}$ the dual summation variable, we find

$$
\mathcal{Z}_{\mathrm{G}}^{(p, q)}(\mathcal{N}, C)=\mathcal{D}^{1 / 2}|\mathcal{F}|^{2} \sum_{\left(n^{\Lambda}, r^{\Lambda}\right) \in \Gamma_{m}} e^{-\tilde{S}},
$$

where $\mathcal{D} \equiv \operatorname{det}\left(-\frac{2 q}{p} \operatorname{Im} \mathcal{N}\right)$. The dual action separates into

$$
\begin{aligned}
\tilde{S}= & \mathrm{i} \pi k\left(p_{R}^{\Lambda} \mathcal{N}_{\Lambda \Sigma} p_{R}^{\Sigma}-p_{L}^{\Lambda} \overline{\mathcal{N}}_{\Lambda \Sigma} p_{L}^{\Sigma}\right)+2 \pi \mathrm{i} k \bar{\omega}_{\Lambda} p_{R}^{\Lambda}+2 \pi \mathrm{i} k q \phi_{\Lambda}\left(p_{L}^{\Lambda}+p_{R}^{\Lambda}\right) \\
& -\frac{\pi k}{2} \bar{\omega}_{\Lambda} \operatorname{Im} \mathcal{N}^{\Lambda \Sigma}\left(\omega_{\Sigma}-\bar{\omega}_{\Sigma}\right)
\end{aligned}
$$

where the vectors $p_{L}, p_{R} \in \Gamma_{m} /(2|k|)$ are the following linear combinations of $n^{\Lambda}$ and $r^{\Lambda}$,

$$
\begin{aligned}
& p_{R}^{\Lambda}=\frac{1}{2}\left(q^{-1}+q\right) n^{\Lambda}-\left(p^{-1} r^{\Lambda}+q \theta^{\Lambda}\right), \\
& p_{L}^{\Lambda}=\frac{1}{2}\left(q^{-1}-q\right) n^{\Lambda}+\left(p^{-1} r^{\Lambda}+q \theta^{\Lambda}\right) .
\end{aligned}
$$

For later reference, we note that the last term in (2.27) may be rewritten in terms of the real periods (2.6) of the $C$-field as

$$
\frac{\pi k}{2} \bar{\omega}_{\Lambda} \operatorname{Im} \mathcal{N}^{\Lambda \Sigma}\left(\omega_{\Sigma}-\bar{\omega}_{\Sigma}\right)=\mathrm{i} \pi k \zeta^{\Lambda}\left(\tilde{\zeta}_{\Lambda}-\mathcal{N}_{\Lambda \Sigma} \zeta^{\Sigma}\right)
$$


Expressing the sum over $\left(n^{\Lambda}, r^{\Lambda}\right)$ as a sum over $p_{L}, p_{R}$ in suitably shifted lattices, the total partition function then decomposes as ([35], Thm. E.1 $)^{13}$

$$
\mathcal{Z}_{\mathrm{G}}^{(p, q)}(\mathcal{N}, C)=\mathcal{D}^{1 / 2} \sum_{\substack{\mu_{p} \in\left(\Gamma_{m} / p\right) / \Gamma_{m} \\ \mu_{q} \in\left(\Gamma_{m} / q\right) / \Gamma_{m}}} \mathcal{Z}_{\Theta, \mu_{p}+\mu_{q}}^{(k)}(\mathcal{N}, 0) \overline{\mathcal{Z}_{\Theta, \mu_{p}-\mu_{q}}^{(k)}(\mathcal{N}, C)},
$$

analogous to the sum of products of holomorphic and anti-holomorphic conformal blocks appearing in the partition function of a compact scalar field on a two-torus. The "holomorphic conformal block" $\mathcal{Z}_{\Theta, \mu}^{(k)}$ appearing in $(2.31)$ is given by ${ }^{14}$

$$
\mathcal{Z}_{\Theta, \mu}^{(k)}(\mathcal{N}, C)=\mathcal{F} \sum_{n \in \Gamma_{m}+\mu+\theta} \mathbf{E}\left(\frac{k}{2}\left(n^{\Lambda} \overline{\mathcal{N}}_{\Lambda \Sigma} n^{\Sigma}+\theta^{\Lambda} \phi_{\Lambda}\right)+k\left(\omega_{\Lambda}-\phi_{\Lambda}\right) n^{\Lambda}+\frac{\mathrm{i} k}{4} \omega_{\Lambda} \operatorname{Im} \mathcal{N}^{\Lambda \Sigma}\left(\omega_{\Sigma}-\bar{\omega}_{\Sigma}\right)\right)
$$

In this expression, both the modulus and phase of the prefactor $\mathcal{F}$ are unspecified at this point, but depend only on the complex structure of $\mathcal{X}$ and string coupling. Up to this normalization ambiguity, $\mathcal{Z}_{\mathrm{G}}^{(k)} \equiv \mathcal{Z}_{\Theta, \mu}^{(k)}$ is then the sought-for partition function of a selfdual three-form on the worldvolume $\mathcal{X}$, in the weak coupling limit, and in the topological sector with quadratic refinement $\Theta=(\theta, \phi)$. The shift vector $\mu$ runs over the $|k|^{b_{3}(\mathcal{X})}$ elements in $\left(\Gamma_{m} /|k|\right) / \Gamma_{m}$, so for $|k| \geq 1$ the partition function is vector-valued. While the derivation of (2.32) from holomorphic factorization has assumed that $2 \Theta \in \Gamma$, the result (2.32) is well-defined for any $\Theta$, allowing us the relax the assumption that $\Theta$ was half-integer. We reiterate that $\mathcal{Z}_{\mathrm{G}}^{(k)}$ differs from the fivebrane instanton partition function in the weak coupling limit $\mathcal{Z}_{\mathrm{NS5}-\mathrm{G}}^{(k)}$ to be computed in section 5, although the difference is inessential at the level of the present discussion.

\subsection{Chiral partition function and Gaussian theta series}

For $k=1$, the partition function of a Gaussian self-dual three-form (2.32) is recognized as a Siegel theta series of rank $b_{3}(\mathcal{X}) / 2$ with in general, real characteristics $\Theta=\left(\theta^{\Lambda}, \phi_{\Lambda}\right)$, evaluated at the period matrix $\overline{\mathcal{N}}$ on the Siegel upper-half plane. More precisely, defining the standard holomorphic Siegel theta series as

$$
\vartheta_{\text {Siegel }}\left[\begin{array}{l}
\theta \\
\phi
\end{array}\right]\left(\overline{\mathcal{N}}, \omega_{\Lambda}\right)=\sum_{n \in \Gamma_{m}+\theta} \mathbf{E}\left(\frac{1}{2} n^{\Lambda} \overline{\mathcal{N}}_{\Lambda \Sigma} n^{\Sigma}+\left(\omega_{\Lambda}-\phi_{\Lambda}\right) n^{\Lambda}\right)
$$

we have, independently of $\mu$,

$$
\mathcal{Z}_{\Theta, \mu}^{(1)}(\mathcal{N}, C)=\mathcal{F} \mathbf{E}\left(\frac{1}{2} \theta^{\Lambda} \phi_{\Lambda}+\frac{\mathrm{i}}{4} \omega_{\Lambda} \operatorname{Im} \mathcal{N}^{\Lambda \Sigma}\left(\omega_{\Sigma}-\bar{\omega}_{\Sigma}\right)\right) \vartheta_{\text {Siegel }}\left[\begin{array}{c}
\theta \\
\phi
\end{array}\right]\left(\overline{\mathcal{N}}, \omega_{\Lambda}\right)
$$

\footnotetext{
${ }^{13}$ Eq. (2.31) holds under the assumptions that $\Theta$ is half-integer, $p q=p(\bmod 2)$ and $\operatorname{gcd}(p, q)=1$, in particular $q$ is odd. More generally, the partition function can always be decomposed into a sum of products of level $p q / 2 m^{2}$ theta series, where $m=\operatorname{gcd}(p, q)$. The quadratic refinement $\sigma(H)$ in $(2.20)$ is crucial in ensuring that a single characteristics $\Theta$ appears in the sum [64].

${ }^{14}$ This matches eq. E.9 in [35] upon identifying $\left(T, a_{1}, a_{2}, \gamma, n^{I}, w^{I}\right)$ there with $\left(\overline{\mathcal{N}}, \zeta, \tilde{\zeta}, \mu, n^{\Lambda}, r^{\Lambda}+p q n^{\Lambda}\right)$ here.
} 
More generally, for $|k|>1$ eq. (2.32) is a level $k / 2$ generalization of the Siegel theta series. Using (2.30), it may be usefully rewritten as

$$
\mathcal{Z}_{\Theta, \mu}^{(k)}(\mathcal{N}, C)=\mathcal{F} \sum_{n \in \Gamma_{m}+\mu+\theta} \mathbf{E}\left(\frac{k}{2}\left(\zeta^{\Lambda}-n^{\Lambda}\right) \overline{\mathcal{N}}_{\Lambda \Sigma}\left(\zeta^{\Sigma}-n^{\Sigma}\right)+k\left(\tilde{\zeta}_{\Lambda}-\phi_{\Lambda}\right) n^{\Lambda}+\frac{k}{2}\left(\theta^{\Lambda} \phi_{\Lambda}-\zeta^{\Lambda} \tilde{\zeta}_{\Lambda}\right)\right)
$$

Under translations by a vector $H=\left(\eta^{\Lambda}, \tilde{\eta}_{\Lambda}\right) \in \Gamma$ on the torus $\mathcal{T}$, i.e. large gauge transformations of the $C$-field, the partition function (2.35) satisfies the twisted periodicity property $^{15}$

$$
\mathcal{Z}_{\Theta, \mu}^{(k)}(\mathcal{N}, C+H)=\left(\sigma_{\Theta}(H)\right)^{k} \mathbf{E}\left(\frac{k}{2}\left(\eta^{\Lambda} \tilde{\zeta}_{\Lambda}-\tilde{\eta}_{\Lambda} \zeta^{\Lambda}\right)\right) \mathcal{Z}_{\Theta, \mu}^{(k)}(\mathcal{N}, C)
$$

Thus, $\mathcal{Z}_{\Theta, \mu}^{(k)}$ is not a function on $\mathcal{T}$, but rather a section of $\left(\mathcal{C}_{\Theta}\right)^{k}$, where $\mathcal{C}_{\Theta}$ is the circle bundle over $\mathcal{T}$ with first Chern class equal to the Kähler class $\omega_{\mathcal{T}}$ in (2.8), and whose sections satisfy the twisted periodicity property (2.36) for $k=1$ [28]. It is important to stress that $\mathcal{C}_{\Theta}$ and $\mathcal{C}_{\Theta^{\prime}}$ are non-isomorphic bundles unless $\Theta-\Theta^{\prime} \in \Gamma$. Yet, the theta series for different values of the characteristics are related by a translation along the torus,

$$
\mathcal{Z}_{\Theta, \mu}^{(k)}(\mathcal{N}, C)=\mathbf{E}\left(\frac{k}{2}\left(\left\langle\Theta, \Theta^{\prime}\right\rangle+\left\langle C, \Theta-\Theta^{\prime}\right\rangle\right)\right) \mathcal{Z}_{\Theta^{\prime}, \mu}^{(k)}\left(\mathcal{N}, C+\Theta^{\prime}-\Theta\right) .
$$

Moreover, thanks to its Gaussian character, the flux partition function is actually a holomorphic section of $\left(\mathcal{L}_{\Theta}\right)^{k}$, where $\mathcal{L}_{\Theta}$ is the line bundle with circle bundle $\mathcal{L}_{\Theta}^{\circ}=\mathcal{C}_{\Theta}$. Indeed, $\mathcal{Z}_{\Theta, \mu}^{(k)}$ is annihilated by the covariant anti-holomorphic derivative

$$
\left(\frac{\partial}{\partial \bar{\omega}_{\Lambda}}-\frac{\pi k}{2} \operatorname{Im} \mathcal{N}^{\Lambda \Sigma} \omega_{\Sigma}\right) \mathcal{Z}_{\Theta, \mu}^{(k)}\left(\mathcal{N}, \omega_{\Lambda}, \bar{\omega}_{\Lambda}\right)=0
$$

By the usual index theorem and Kodaira vanishing argument [28], the line bundle $\mathcal{L}_{\Theta}^{k}$ admits exactly $|k|^{b_{3}(\mathcal{X}) / 2}$ holomorphic sections, corresponding to the possible values of $\mu \in\left(\Gamma_{m} /|k|\right) / \Gamma_{m}$. Physically, this is the familiar degeneracy of the Landau levels for a particle moving on a $b_{3}(\mathcal{X})$-dimensional torus with $k$ units of magnetic flux.

Having described the behavior of the flux partition function (2.35) under large gauge transformations, we now turn to its behavior under monodromies in complex structure moduli space $\mathcal{M}_{c}(\mathcal{X})$. Under a monodromy $M$, the period matrix transforms by fractional linear transformations (2.13), the $C$ field transforms as $C \mapsto \rho(M) \cdot C$ and the characteristics $\Theta$ transform as (2.24), leaving the non-chiral partition function $\mathcal{Z}_{\mathrm{G}}^{(p, q)}$ invariant. In contrast, the modular properties of the Siegel theta series imply that the chiral fivebrane partition function $\mathcal{Z}_{\Theta, \mu}^{(k)}$ is mapped to a linear superposition of $\mathcal{Z}_{\Theta^{\prime}, \mu^{\prime}}^{(k)}$ with $\mu^{\prime}$ ranging over $\left(\Gamma_{m} /|k|\right) / \Gamma_{m}$, and fixed characteristics $\Theta^{\prime}$. Thus, the bundle where $\mathcal{Z}_{\Theta, \mu}^{(k)}$ takes values is also non-trivially fibered over $\mathcal{M}_{c}(\mathcal{X})$, as will be further elaborated upon in section 3 . Specifically, under symplectic transformations the level $k / 2$ theta series $\vartheta_{(\theta, \phi), \mu}^{(k)} \equiv \mathcal{F}^{-1} \mathcal{Z}_{\Theta, \mu}^{(k)}$ transforms as [35]:

\footnotetext{
${ }^{15}$ Note that the defining property $(2.21)$ of the quadratic refinement is crucial in ensuring the consistency of this periodicity condition.
} 
1. for $\rho(M)=\left(\begin{array}{cc}\mathcal{A}^{-T} & 0 \\ 0 & \mathcal{A}\end{array}\right)$ :

$$
\vartheta_{(\theta, \phi), \mu}^{(k)}\left(\mathcal{A N} \mathcal{A}^{T}, \zeta, \tilde{\zeta}\right)=\vartheta_{\left(\mathcal{A}^{T} \theta, \mathcal{A}^{-1} \phi\right), \mathcal{A}^{T} \mu}^{(k)}\left(\mathcal{N}, \mathcal{A}^{T} \zeta, \mathcal{A}^{-1} \tilde{\zeta}\right)
$$

2. for $\rho(M)=\left(\begin{array}{ll}1 & 0 \\ \mathcal{B} & 1\end{array}\right)$, where $\mathcal{B}$ is a symmetric integer matrix,

$$
\begin{aligned}
\vartheta_{(\theta, \phi), \mu}^{(k)}(\mathcal{N}+\mathcal{B}, \zeta, \tilde{\zeta})= & \mathbf{E}\left(-\frac{k}{4} \mathcal{B}_{\Lambda \Lambda}\left(\theta^{\Lambda}+2 \mu^{\Lambda}\right)+\frac{k}{2} \mathcal{B}_{\Lambda \Sigma} \mu^{\Lambda} \mu^{\Sigma}\right) \\
& \times \vartheta_{\left(\theta^{\Lambda}, \phi_{\Lambda}-\mathcal{B}_{\Lambda \Sigma} \theta^{\Sigma}-\frac{1}{2} \mathcal{B}_{\Lambda \Lambda}\right), \mu}^{(k)}(\mathcal{N}, \zeta, \tilde{\zeta}-\mathcal{B} \zeta)
\end{aligned}
$$

3. for $\rho(M)=\left(\begin{array}{cc}0 & -1 \\ 1 & 0\end{array}\right)$,

$$
\vartheta_{(\theta, \phi), \mu}^{(k)}\left(-\mathcal{N}^{-1}, \zeta, \tilde{\zeta}\right)=\frac{\sqrt{\operatorname{det}(-\mathrm{i} \mathcal{N})}}{k^{b_{3}(\mathcal{X}) / 2}} \sum_{\mu^{\prime} \in\left(\Gamma_{e} /|k|\right) / \Gamma_{e}} \mathbf{E}\left(-k \mu^{\prime} \mu\right) \vartheta_{(-\phi, \theta), \mu^{\prime}}^{(k)}(\mathcal{N},-\tilde{\zeta}, \zeta) .
$$

\subsection{Non-Gaussian theta series and wave-functions}

Our discussion of the topological nature of the chiral partition function thus far was based on the weak coupling result (2.35). However, as explained in section 1.3, we expect that upon including the effects of non-Abelian dynamics and non-linearities on the fivebrane world-volume, the exact NS5-brane partition function $\mathcal{Z}_{\mathrm{NS} 5}^{(k)}$ will continue to take values in the same bundle. With this in mind, we note that the most general solution to the periodicity conditions (2.36) can be written as a non-Gaussian theta series

$$
\mathcal{Z}_{\Theta, \mu}^{(k), \mathrm{NG}}\left(\mathcal{N}, \zeta^{\Lambda}, \tilde{\zeta}_{\Lambda}\right)=\sum_{n \in \Gamma_{m}+\mu+\theta} \Psi_{\mathbb{R}}^{k, \mu}\left(\zeta^{\Lambda}-n^{\Lambda}\right) \mathbf{E}\left(k\left(\tilde{\zeta}_{\Lambda}-\phi_{\Lambda}\right) n^{\Lambda}+\frac{k}{2}\left(\theta^{\Lambda} \phi_{\Lambda}-\zeta^{\Lambda} \tilde{\zeta}_{\Lambda}\right)\right)
$$

where $\Psi_{\mathbb{R}}^{k, \mu}\left(\zeta^{\Lambda}\right)$ will also depend on the complex structure moduli and the string coupling. In general, unlike its Gaussian counterpart, eq. (2.42) is a section of the circle bundle $\mathcal{C}_{\Theta}^{k}=\left(\mathcal{L}_{\Theta}^{\circ}\right)^{k}$ but not a holomorphic section of the line bundle $\mathcal{L}_{\Theta}^{k}$.

In order to satisfy the same transformation law as (2.35) under monodromies in $\mathcal{M}_{c}(\mathcal{X})$, $\Psi_{\mathbb{R}}^{k, \mu}\left(\zeta^{\Lambda}\right)$ should be invariant under the combined action of fractional linear transformations (2.13) on the period matrix $\mathcal{N}$ and under monodromies acting in the metaplectic representation $\rho_{m}(M)$. This implies that $\Psi_{\mathbb{R}}^{k, \mu}\left(\zeta^{\Lambda}\right)$ should be viewed as the wave function of a certain state $\left|\Psi^{k, \mu}\right\rangle$ in the Hilbert space $\mathcal{H}$ of square-integrable functions on $H^{3}(\mathcal{X}, \mathbb{R})$. To spell this out, consider first a specific state $\left|\Psi^{\Gamma_{m}, k, \mu}\right\rangle \in \mathcal{H}$, conveniently represented as follows in terms of its wave function $\Psi_{\mathbb{R}}^{\Gamma_{m}, k, \mu}\left(\zeta^{\Lambda}\right)$ in the $\zeta$-representation (corresponding to the real polarization for the topological wave function as further explained in the next subsection):

$$
\Psi_{\mathbb{R}}^{\Gamma_{m}, k, \mu}\left(\zeta^{\Lambda}\right)=e^{-\mathrm{i} \pi k \theta^{\Lambda} \phi_{\Lambda}} \sum_{n \in \Gamma_{m}+\mu+\theta} \delta\left(\zeta^{\Lambda}-n^{\Lambda}\right) e^{2 \pi \mathrm{i} k \phi_{\Lambda} n^{\Lambda}}
$$


We further introduce two sets of operators, $T^{\Lambda}$ and $\tilde{T}_{\Lambda}$, acting on $\mathcal{H}$. Their action on an arbitrary wave function in the real polarization reads

$$
T^{\Lambda} \cdot \Psi_{\mathbb{R}}\left(\zeta^{\Lambda}\right)=2 \pi k \zeta^{\Lambda} \Psi_{\mathbb{R}}\left(\zeta^{\Lambda}\right), \quad \tilde{T}_{\Lambda} \cdot \Psi_{\mathbb{R}}\left(\zeta^{\Lambda}\right)=-\mathrm{i} \partial_{\zeta^{\Lambda}} \Psi_{\mathbb{R}}\left(\zeta^{\Lambda}\right),
$$

so that they satisfy the Heisenberg algebra

$$
\left[T^{\Lambda}, \tilde{T}_{\Sigma}\right]=2 \pi \mathrm{i} k \delta_{\Sigma}^{\Lambda}
$$

Our main observation is then that the non-Gaussian theta series (2.42) can be rewritten as a matrix element involving the two states introduced above:

$$
\mathcal{Z}_{\Theta, \mu}^{(k), \mathrm{NG}}(\mathcal{N}, C)=\left\langle\Psi^{\Gamma_{m}, k, \mu}\left|e^{-\mathrm{i}\left(\zeta^{\Lambda} \tilde{T}_{\Lambda}-\tilde{\zeta}_{\Lambda} T^{\Lambda}\right)}\right| \Psi^{k, \mu}\right\rangle .
$$

In particular, the Gaussian chiral partition function (2.35) is recovered by choosing the state $\left|\Psi^{k, \mu}\right\rangle$ as follows:

$$
\Psi_{\mathbb{R}}^{k, \mu}\left(\zeta^{\Lambda}\right)=\mathcal{F} e^{\pi \mathrm{i} k \zeta^{\Lambda} \overline{\mathcal{N}}_{\Lambda \Sigma} \zeta^{\Sigma}}
$$

where $\mathcal{F}$ is the normalization factor in $(2.35)$.

In section 5 we will find that the relation (2.46) indeed captures the correct partition function $\mathcal{Z}_{\text {NS5 }}^{(k)}$ of the NS5-brane, provided we identify $\left|\Psi^{k, \mu}\right\rangle$ with a particular state associated with the B-model topological string, for which the Gaussian wave function (2.47) corresponds to the weak coupling approximation. To pave the way for these developments, we will in the next section introduce some relevant background material on topological strings.

\subsection{Topological wave functions in different polarizations}

In this section we review in some detail various important properties of topological string wave functions that will play an important role in section 5 . We begin the analysis from the point of view of the topological B-model, while towards the end discussing the map to the A-model. A novel observation of this section is that the chiral partition function $\mathcal{Z}_{\Theta, \mu}^{(k)}$ in (2.35), restricted to $k=1$, can be represented as the state (2.43) in the so called "Weil polarization" as shown in (2.73).

\subsubsection{Griffiths polarization}

Recall that in the topological B-model on $\mathcal{X}$, the partition function is defined as the generating function of the genus $g$ correlation functions $C_{a_{1} \cdots a_{n}}^{(g)}$ of $n$ chiral fields [45]:

$$
\Psi_{\mathrm{BCOV}}(z, \bar{z} ; \lambda, x)=\lambda^{\frac{\chi(\mathcal{X})}{24}-1} \exp \left(\sum_{g=0}^{\infty} \sum_{n=0}^{\infty} \frac{1}{n !} \lambda^{2 g-2} C_{a_{1} \cdots a_{n}}^{(g)}(z, \bar{z}) x^{a_{1}} \cdots x^{a_{n}}\right) .
$$

The correlation functions $C_{a_{1} \cdots a_{n}}^{(g)}$, which are taken to vanish for $2 g-2+n \leq 0$, are global sections of the vector bundle $\left(T^{*}\right)^{n} \otimes \mathcal{L}^{2-2 g}$ over the complex structure moduli space $\mathcal{M}_{c}(\mathcal{X})$. Here, $T^{*}$ is the holomorphic cotangent bundle of $\mathcal{M}_{c}(\mathcal{X})$ and $\mathcal{L}$ is the line bundle over $\mathcal{M}_{c}(\mathcal{X})$ in which the holomorphic three-form $\Omega_{3,0}$ is valued. In particular, under rescalings $\Omega_{3,0} \mapsto e^{f} \Omega_{3,0}$, the correlation functions transform as $C_{a_{1} \cdots a_{n}}^{(g)} \mapsto e^{(2-2 g) f} C_{a_{1} \cdots a_{n}}^{(g)}$. 
As a result, $\Psi_{\mathrm{BCOV}}$ is a section of the line bundle ${ }^{16} \mathcal{L}^{\frac{\chi(\mathcal{X})}{24}-1}$, provided $\lambda$ transforms as a section of $\mathcal{L}$.

Moreover, $\Psi_{\mathrm{BCOV}}$ satisfies the holomorphic anomaly conditions obtained in [45], eq. 3.17-18. As explained in [66], it is illuminating to rescale $x^{a}$ and $\Psi_{\mathrm{BCOV}}$ as $^{17}$

$$
\Psi_{\mathrm{G}}\left(z, \bar{z} ; \lambda^{-1}, x\right)=e^{f_{1}(z)} \Psi_{\mathrm{BCOV}}(z, \bar{z} ; \lambda, \lambda x),
$$

where $f_{1}$ is (locally) a holomorphic function determined from the factorization of the oneloop vacuum amplitude $F_{1}$ by

$$
F_{1}=\log \left[e^{f_{1}(z)+\bar{f}_{1}(\bar{z})} / \sqrt{M(z, \bar{z})}\right],
$$

where

$$
M(z, \bar{z})=|g| e^{-2\left(\frac{b_{3}}{4}-\frac{\chi(\mathcal{X})}{24}+1\right) \mathcal{K}}, \quad|g|=\operatorname{det}\left(g_{a \bar{b}}\right) .
$$

More accurately, $e^{f_{1}(z)}$ is a section of $\mathcal{L}^{1-\frac{\chi(\mathcal{X})}{24}+\frac{b_{3}}{4}} \otimes K_{c}^{1 / 2}$, where $K_{c}$ is the canonical bundle of $\mathcal{M}_{c}$, locally trivialized by the section $d z^{1} \wedge \cdots \wedge d z^{h_{2,1}}$. Thus, $f_{1}$ transforms under holomorphic change of coordinates $z \mapsto z^{\prime}(z)$ and rescaling $\left(\lambda^{-1}, x^{a}\right) \mapsto e^{-f}\left(\lambda^{-1}, x^{a}\right)$ according to

$$
f_{1} \mapsto f_{1}+\left(\frac{b_{3}}{4}-\frac{\chi(\mathcal{X})}{24}+1\right) f+\frac{1}{2} \log \operatorname{det}\left(\partial z / \partial z^{\prime}\right) .
$$

This implies that the rescaled amplitude $\Psi_{\mathrm{G}}$ and its covariant derivative $\nabla_{a} \Psi_{\mathrm{G}}$ transform as $\mathcal{L}^{b_{3} / 4}$-valued holomorphic half-densities on $\mathcal{M}_{c}(\mathcal{X})$, namely

$$
\Psi_{\mathrm{G}} \mapsto \sqrt{\operatorname{det}\left(\partial z / \partial z^{\prime}\right)} e^{b_{3} f / 4} \Psi_{\mathrm{G}} .
$$

In terms of $\Psi_{\mathrm{G}}$, the holomorphic anomaly equations of [45] take the more appealing form $[66,67]$

$$
\begin{array}{r}
{\left[\frac{\partial}{\partial \bar{z}^{a}}-\frac{1}{2} e^{2 \mathcal{K}} \bar{C}_{\bar{a} \bar{b} \bar{c}} g^{b \bar{b}} g^{c \bar{c}} \frac{\partial^{2}}{\partial x^{b} \partial x^{c}}-g_{\bar{a} b} x^{j} \frac{\partial}{\partial \lambda^{-1}}\right] \Psi_{\mathrm{G}}=0,} \\
{\left[\nabla_{a}-\Gamma_{a b}^{c} x^{b} \frac{\partial}{\partial x^{c}}-\frac{1}{2} \partial_{a} \log |g|-\lambda^{-1} \frac{\partial}{\partial x^{i}}+\frac{1}{2} C_{a b c} x^{b} x^{c}\right] \Psi_{\mathrm{G}}=0,}
\end{array}
$$

where $\nabla_{a}$ is the covariant derivative

$$
\nabla_{a}=\frac{\partial}{\partial z^{a}}+\partial_{a} \mathcal{K}\left(x^{b} \frac{\partial}{\partial x^{b}}+\lambda^{-1} \frac{\partial}{\partial \lambda^{-1}}+\frac{b_{3}}{4}\right) .
$$

The holomorphic anomaly equations (2.54) then guarantee that the inner product

$$
\begin{aligned}
\langle\Psi \mid \Psi\rangle= & \int \mathrm{d} x^{a} \mathrm{~d} \bar{x}^{\bar{a}} \mathrm{~d} \lambda^{-1} \mathrm{~d} \bar{\lambda}^{-1} \sqrt{|g|} e^{-\frac{b_{3}}{4} \mathcal{K}} \\
& \exp \left(-e^{-\mathcal{K}} x^{a} g_{a \bar{b}} \bar{x}^{\bar{b}}+e^{-\mathcal{K}^{-1}} \bar{\lambda}^{-1}\right) \Psi_{\mathrm{G}}^{*}\left(\bar{z}, z ; \bar{\lambda}^{-1}, \bar{x}\right) \Psi_{\mathrm{G}}\left(z, \bar{z} ; \lambda^{-1}, x\right)
\end{aligned}
$$

is well defined, and (at least formally) independent of the complex structure moduli.

\footnotetext{
${ }^{16}$ As mentioned at the end of section 1.4, the definition of fractional power $\mathcal{L}^{\chi / 24}$ requires a homomorphism $M \mapsto \mathbf{E}(\kappa(M))$ from the monodromy group to the group of 24:th roots of unity. In the present case, this homomorphism is just the multiplier system of the one-loop amplitude $e^{-f_{1}} / \sqrt{J_{\mathrm{G}}}$, where $f_{1}$ and $J_{\mathrm{G}}$ are defined in (2.50) and below (2.68).

${ }^{17}$ The index $\mathrm{G}$ refers to the Griffiths complex structure, as will become clear momentarily.
} 
As explained in [66-69], the above equations may be interpreted as the fact that $\Psi_{\mathrm{G}}$ is the wave-function for a particular state $|\Psi\rangle \equiv\left|\Psi^{\text {top }}\right\rangle$ in the Hilbert space $\mathcal{H}$ quantizing the symplectic space $H^{3}(\mathcal{X}, \mathbb{R})$ in a complex polarization determined by the Griffiths complex structure. Indeed, for a given choice of the holomorphic $\Omega_{3,0}$ on $\mathcal{X}$, any 3 -form $C \in$ $H^{3}(\mathcal{X}, \mathbb{R})$ admits the Hodge decomposition

$$
\sqrt{2 \pi} C=\lambda^{-1} \Omega_{3,0}+x^{a} D_{a} \Omega_{3,0}+\bar{x}^{\bar{a}} D_{\bar{a}} \bar{\Omega}_{3,0}+\bar{\lambda}^{-1} \bar{\Omega}_{3,0},
$$

where $\lambda^{-1}, x^{a}$ are complex coordinates for the Griffiths complex structure on $H^{3}(\mathcal{X}, \mathbb{C})$ and the normalization factor $\sqrt{2 \pi}$ is inserted for later convenience. The solutions of $(2.54)$ may be written as the overlap

$$
\Psi_{\mathrm{G}}\left(z, \bar{z} ; \lambda^{-1}, x\right)={ }_{(z, \bar{z})}\left\langle\lambda^{-1}, x \mid \Psi\right\rangle,
$$

where ${ }_{(z, \bar{z})}\left\langle\lambda^{-1}, x\right|$ is a basis of coherent states diagonalizing the action of the operators quantizing the components $\lambda^{-1}$ and $x^{a}$ in (2.57). The holomorphic equations (2.54) then reflect the unitary transformation undergone by the coherent states under changes of complex structure.

\subsubsection{Weil polarization}

Alternatively, one may choose to diagonalize the operators quantizing $\bar{\lambda}^{-1}$ and $x^{a}$, which are complex coordinates for the Weil complex structure on $H^{3}(\mathcal{X}, \mathbb{C})$. The new topological wave function in the "Weil complex polarization"

$$
\Psi_{\mathrm{W}}\left(z, \bar{z} ; \bar{\lambda}^{-1}, x\right)={ }_{(z, \bar{z})}\left\langle\bar{\lambda}^{-1}, x \mid \Psi\right\rangle
$$

is obtained from the topological wave function in the "Griffiths complex polarization" (2.58) by Fourier transforming over $\lambda^{-1}$,

$$
\Psi_{\mathrm{W}}\left(z, \bar{z} ; \bar{\lambda}^{-1}, x\right)=\int \mathrm{d} \lambda^{-1} \exp \left(\mathrm{i} e^{-\mathcal{K}}(\lambda \bar{\lambda})^{-1}\right) \Psi_{G}\left(z, \bar{z} ; \lambda^{-1}, x\right),
$$

such that the inner product is now given by a positive definite Gaussian kernel,

$$
\begin{aligned}
\langle\Psi \mid \Psi\rangle= & \int \mathrm{d} x^{a} \mathrm{~d} \bar{x}^{\bar{a}} \mathrm{~d} \lambda^{-1} \mathrm{~d} \bar{\lambda}^{-1} \sqrt{|g|} e^{-\frac{b_{3}}{4} \mathcal{K}} \\
& \exp \left(-e^{-\mathcal{K}} x^{a} g_{a \bar{b}} \bar{x}^{\bar{b}}-e^{-\mathcal{K}} \lambda^{-1} \bar{\lambda}^{-1}\right) \Psi_{W}^{*}\left(\bar{z}, z ; \lambda^{-1}, \bar{x}\right) \Psi_{W}\left(z, \bar{z} ; \bar{\lambda}^{-1}, x\right) .
\end{aligned}
$$

It is in principle straightforward to work out the analog of (2.54) for this new polarization. Since $\Psi_{\mathrm{G}}$ transformed as (2.53) under holomorphic change of coordinates and rescalings, $\Psi_{\mathrm{W}}$ must now transform as a $\mathcal{L}^{b_{3} / 4-1}$-valued holomorphic half-density,

$$
\Psi_{\mathrm{W}} \mapsto \sqrt{\operatorname{det}\left(\partial z / \partial z^{\prime}\right)} e^{\left(\frac{b_{3}}{4}-1\right) f} \Psi_{\mathrm{W}} .
$$

Importantly, the relations (2.53), (2.62) are properties of the coherent state bases ${ }_{(z, \bar{z})}\left\langle\lambda^{-1}, x\right|$ and ${ }_{(z, \bar{z})}\left\langle\bar{\lambda}^{-1}, x\right|$, and hold for an arbitrary state $|\Psi\rangle \in \mathcal{H}$, not only $\left|\Psi^{\text {top }}\right\rangle \in \mathcal{H}$. Moreover, both $\Psi_{\mathrm{G}}$ and $\Psi_{\mathrm{W}}$ depend on the complex structure of $\mathcal{X}$, but are independent of any choice of symplectic basis on $H^{3}(\mathcal{X}, \mathbb{R})$. 


\subsubsection{Real polarization and intertwiners}

However, at the expense of fixing a symplectic basis $\alpha_{\Lambda}, \beta^{\Lambda}$ of $H^{3}(\mathcal{X}, \mathbb{R})$, it becomes possible to express the state $|\Psi\rangle$ in a "background independent" way, i.e. independent of the complex structure on $\mathcal{X}$. Indeed, one may expand $C \in H^{3}(\mathcal{X}, \mathbb{R})$ as in $(2.6)$,

$$
C=\zeta^{\Lambda} \alpha_{\Lambda}-\tilde{\zeta}_{\Lambda} \beta^{\Lambda},
$$

where $\zeta^{\Lambda}$ and $\tilde{\zeta}_{\Lambda}$ are real Darboux coordinates on $H^{3}(\mathcal{X}, \mathbb{R})$, and express $|\Psi\rangle$ on a basis of wave functions diagonalizing the operators associated to $\zeta^{\Lambda}$, say. The resulting wave function

$$
\Psi_{\mathbb{R}}\left(\zeta^{\Lambda}\right)=\left\langle\zeta^{\Lambda} \mid \Psi\right\rangle
$$

in the so called "real polarization", is locally independent of the complex structure of $\mathcal{X}$, but transforms according to the metaplectic representation $\rho_{m}$ under changes of symplectic basis, in particular under monodromies in $\mathcal{M}_{c}(\mathcal{X})$. On square-integrable functions $\Psi_{\mathbb{R}}\left(\zeta^{\Lambda}\right)$, the metaplectic representation acts according to

$$
\begin{aligned}
{\left[\rho_{m}\left(\left(\begin{array}{cc}
\mathcal{A}^{-T} & 0 \\
0 & \mathcal{A}
\end{array}\right)\right) \cdot \Psi_{\mathbb{R}}\right]\left(\zeta^{\Lambda}\right) } & =\Psi_{\mathbb{R}}\left(\mathcal{A}^{T} \zeta^{\Lambda}\right), \\
{\left[\rho_{m}\left(\left(\begin{array}{cc}
1 & 0 \\
\mathcal{B} & 1
\end{array}\right)\right) \cdot \Psi_{\mathbb{R}}\right]\left(\zeta^{\Lambda}\right) } & =\mathbf{E}\left(\frac{1}{2} \mathcal{B}_{\Lambda \Sigma} \zeta^{\Lambda} \zeta^{\Sigma}\right) \Psi_{\mathbb{R}}\left(\zeta^{\Lambda}\right), \\
{\left[\rho_{m}\left(\left(\begin{array}{cc}
0 & -1 \\
1 & 0
\end{array}\right)\right) \cdot \Psi_{\mathbb{R}}\right]\left(\zeta^{\Lambda}\right) } & =\int \mathbf{E}\left(-k \zeta^{\Lambda} \tilde{\zeta}_{\Lambda}\right) \Psi_{\mathbb{R}}\left(\tilde{\zeta}_{\Lambda}\right) \mathrm{d} \tilde{\zeta}_{\Lambda} .
\end{aligned}
$$

We further note that the metaplectic representation is unitary with respect to the inner product

$$
\langle\Psi \mid \Psi\rangle=\int \Psi_{\mathbb{R}}^{*}\left(\zeta^{\Lambda}\right) \Psi_{\mathbb{R}}\left(\zeta^{\Lambda}\right) \mathrm{d} \zeta^{\Lambda} .
$$

The intertwiner between the real polarization and the Griffiths complex polarization was discussed in $[66,67,69,70]$. Since the Griffiths complex coordinates $\lambda^{-1}, x^{a}$ and the real coordinates $\zeta^{\Lambda}, \tilde{\zeta}_{\Lambda}$ are related classically by

$$
x_{\Lambda} \equiv \tilde{\zeta}_{\Lambda}-\bar{\tau}_{\Lambda \Sigma} \zeta^{\Sigma}=2 \mathrm{i} \operatorname{Im} \tau_{\Lambda \Sigma}\left(\lambda^{-1} X^{\Sigma}+x^{a} D_{a} X^{\Sigma}\right) / \sqrt{2 \pi},
$$

the intertwiner from the real polarization to the Griffiths complex polarization is given by the Gaussian kernel

$$
\Psi_{\mathrm{G}}\left(z, \bar{z} ; \lambda^{-1}, x\right)=\frac{\sqrt{\operatorname{det} \operatorname{Im} \tau}}{\sqrt{J_{\mathrm{G}}}} \int e^{\frac{\pi}{2} x_{\Lambda} \operatorname{Im} \tau^{\Lambda \Sigma} x_{\Sigma}-2 \pi \mathrm{i} x_{\Lambda} \zeta^{\Lambda}-\mathrm{i} \pi \zeta^{\Lambda} \bar{\tau}_{\Lambda \Sigma} \zeta^{\Sigma}} \Psi_{\mathbb{R}}\left(\zeta^{\Lambda}\right) \mathrm{d} \zeta^{\Lambda},
$$

where $J_{\mathrm{G}}=\partial\left(\lambda^{-1}, x^{a}\right) / \partial x^{\Lambda}$ is the Jacobian from the "large phase space" variables $x^{\Lambda}=$ $\operatorname{Im} \tau^{\Lambda \Sigma} x_{\Sigma}$ to the "small phase space" variables $\lambda^{-1}, x^{a}$. A simple computation shows that

$$
\left|J_{\mathrm{G}}\right|^{2}=e^{\frac{b_{3}}{2} \mathcal{K}} \operatorname{det}(\operatorname{Im} \tau) /|g|
$$

therefore $J_{\mathrm{G}}$ transforms as a section of $\mathcal{L}^{-b_{3} / 2} \otimes K_{c}^{-1} \otimes \operatorname{det}_{\mathrm{G}}^{-1}$, where $\operatorname{det}_{\mathrm{G}}$ denotes the line bundle whose sections transform as $w \mapsto \operatorname{det}(\mathcal{C} \tau+\mathcal{D}) w$ under monodromies. On the other hand, as $\Psi_{\mathbb{R}}\left(\zeta^{\Lambda}\right)$ transforms according to the metaplectic representation under 
monodromies, the integral on the r.h.s. of (2.68) is valued in $\overline{\operatorname{det}}_{\mathrm{G}}^{1 / 2}$. As a result, (2.68) indeed transforms as $\mathcal{L}^{b_{3} / 4} \otimes K_{c}^{1 / 2}$ under monodromies. In the special gauge $X^{0}=1$, $X^{a}=z^{a}, J_{\mathrm{G}}$ evaluates to one, thereby identifying the bundles $\mathcal{L}^{-b_{3} / 2} \otimes K_{c}^{-1}$ and $\operatorname{det}_{\mathrm{G}}$. Since the quadratic form $\operatorname{Im} \tau$ has indefinite signature, the intertwiner (2.68) is however only valid formally.

In contrast, the Weil complex coordinates $\left(\bar{\lambda}^{-1}, x^{a}\right)$ are related to the real coordinates $\left(\zeta^{\Lambda}, \tilde{\zeta}_{\Lambda}\right)$ by

$$
\omega_{\Lambda} \equiv \tilde{\zeta}_{\Lambda}-\overline{\mathcal{N}}_{\Lambda \Sigma} \zeta^{\Sigma}=2 \mathrm{i} \operatorname{Im} \mathcal{N}_{\Lambda \Sigma}\left(\lambda^{-1} X^{\Sigma}+\bar{x}^{\bar{a}} D_{\bar{a}} \bar{X}^{\Sigma}\right) / \sqrt{2 \pi}
$$

The intertwiner between the real polarization and the Weil complex polarization is given by the Gaussian kernel [67]

$$
\Psi_{\mathrm{W}}\left(z, \bar{z} ; \bar{\lambda}^{-1}, x\right)=\frac{e^{\mathcal{K} / 2} \sqrt{\operatorname{det}(-\operatorname{Im} \mathcal{N})}}{\sqrt{J_{\mathrm{W}}}} \int e^{-\frac{\pi}{2} \bar{\omega}_{\Lambda} \operatorname{Im} \mathcal{N}^{\Lambda \Sigma} \bar{\omega}_{\Sigma}-2 \pi \mathrm{i} \bar{\omega}_{\Lambda} \zeta^{\Lambda}-\mathrm{i} \pi \zeta^{\Lambda} \mathcal{N}_{\Lambda \Sigma} \zeta^{\Sigma}} \Psi_{\mathbb{R}}\left(\zeta^{\Lambda}\right) \mathrm{d} \zeta^{\Lambda},
$$

where $J_{\mathrm{W}}=\partial\left(\bar{\lambda}^{-1}, x^{a}\right) / \partial \bar{w}^{\Lambda}$ is the Jacobian from $\bar{w}^{\Lambda}=\operatorname{Im} \mathcal{N}^{\Lambda \Sigma} \bar{w}_{\Sigma}$ to $\bar{\lambda}^{-1}, x^{a}$, and the quadratic term in the exponential is now negative definite. Using the relations

$$
\left|J_{\mathrm{W}}\right|^{2}=e^{\frac{b_{3}}{2} \mathcal{K}} \operatorname{det}(-\operatorname{Im} \mathcal{N}) /|g|, \quad \frac{J_{\mathrm{W}}}{J_{\mathrm{G}}}=\frac{e^{-\mathcal{K}}}{\left(\bar{X}^{\Lambda} \operatorname{Im} \tau_{\Lambda \Sigma} \bar{X}^{\Sigma}\right)},
$$

it is apparent that $J_{\mathrm{W}}$ is a section of $\mathcal{L}^{1-\frac{b_{3}}{2}} \otimes \overline{\mathcal{L}}^{-1} \otimes K_{c}^{-1} \otimes{\overline{\operatorname{det}_{\mathrm{W}}}}^{-1}$, where $\operatorname{det}_{\mathrm{W}}$ denotes the line bundle whose sections transform as $w \mapsto \operatorname{det}(\mathcal{C N}+\mathcal{D}) w$ under monodromies. Since the integral on the r.h.s. of (2.71) is valued in $\operatorname{det}_{\mathrm{W}}^{1 / 2},(2.71)$ indeed transforms as a section of $\mathcal{L}^{\frac{b_{3}}{4}-1} \otimes K_{c}^{1 / 2}$. For convenience, we summarize the transformation properties of the various topological wave functions in table 2 on page 64 .

We are now in a position to connect the present discussion to the Gaussian chiral partition function. Let us choose $\Psi_{\mathbb{R}}\left(\zeta^{\Lambda}\right)$ in (2.71) to be the real-polarized wave function associated to the particular state $\left|\Psi^{\Gamma_{m}, k, \mu}\right\rangle \in \mathcal{H}$ in (2.43). Then (the complex conjugate of) the chiral partition function (2.32) for $k=1$ can be written up to a Gaussian prefactor as the wave function of this state in the Weil polarization

$$
\overline{\mathcal{Z}_{\Theta, \mu}^{(1)}(\mathcal{N}, C)}=\overline{\mathcal{F}} e^{-\mathcal{K} / 2} \sqrt{\frac{J_{\mathrm{W}}}{\operatorname{det}(-\operatorname{Im} \mathcal{N})}} e^{\frac{\pi}{2} \omega_{\Lambda} \operatorname{Im} \mathcal{N}^{\Lambda \Sigma} \bar{\omega}_{\Sigma}} \Psi_{\mathrm{W}}^{\Gamma_{m}, 1, \mu}\left(\bar{\omega}_{\Lambda}\right),
$$

which is independent of $\mu$ when $k=1$.

\subsubsection{Holomorphic topological partition function}

Let us now discuss the relation between the topological wave function $\Psi_{\mathrm{BCOV}}$ and the so-called holomorphic topological partition function $F_{\text {hol }}$. For this purpose, we first take the holomorphic limit $\bar{z}^{\bar{a}} \rightarrow \infty$, while keeping $z^{a}$ fixed. We further assume that in this limit, the Griffiths period matrix $\bar{\tau}_{\Lambda \Sigma} \rightarrow \infty$. In this case, the intertwiner (2.68) reduces to a delta function with support on $\zeta^{\Lambda}=-\bar{\tau}^{\Lambda \Sigma} x_{\Sigma}$, so that

$$
\Psi_{\mathrm{G}}\left(x_{\Lambda}\right) \sim J_{\mathrm{G}}^{-1 / 2} \Psi_{\mathbb{R}}\left(\zeta^{\Lambda}\right) .
$$


Let us consider the restriction of the left-hand side to the locus $x^{a}=0, \lambda$ fixed, where $x^{a}, \lambda^{-1}$ are related to $x_{\Lambda}$ by (2.67). On this locus, $\zeta^{\Lambda}$ becomes complexified and equal to $X^{\Lambda} /(\lambda \sqrt{2 \pi})$, which we denote by $\xi^{\Lambda}$. The BCOV amplitude therefore becomes proportional to the topological string wave function in the real polarization $\Psi_{\mathbb{R}}^{\text {top }}$, analytically continued to the complex domain:

$$
\Psi_{\mathrm{BCOV}}(z, \bar{z} \rightarrow \infty ; \lambda, x=0) \sim e^{-f_{1}(z)} \Psi_{\mathbb{R}}^{\mathrm{top}}\left(\xi^{\Lambda}\right)
$$

Thus, defining the holomorphic topological partition function as

$$
\exp \left[F_{\mathrm{hol}}(z, \lambda)\right]=e^{f_{1}(z)} \lim _{\bar{z} \rightarrow \infty}\left[\lambda^{1-\frac{\chi(\mathcal{X})}{24}} \Psi_{\mathrm{BCOV}}(z, \bar{z}, \lambda)\right],
$$

(where the prefactor restores the one-loop vacuum amplitude which was absent from (2.48)), we have, in the Kähler gauge $X^{0}=1$, the relation [70]

$$
\exp \left[F_{\text {hol }}(z, \lambda)\right]=\left(\xi^{0}\right)^{\frac{\chi(\mathcal{X})}{24}-1} \Psi_{\mathbb{R}}^{\text {top }}\left(\xi^{\Lambda}\right), \quad \lambda=\frac{1}{\xi^{0} \sqrt{2 \pi}}, \quad z^{a}=\frac{\xi^{a}}{\xi^{0}} .
$$

This relation will play an important role in section 5.4, where the coordinates $\xi^{\Lambda}$ will be interpreted as Darboux coordinates on twistor space $\mathcal{Z}_{\mathcal{M}}$.

\subsubsection{Topological A-model and Donaldson-Thomas invariants}

So far we have been discussing topological wave functions in the context of the B-model. However, the properties of the topological wave functions discussed in this section hold both for the topological B-model on a $\mathrm{CY}$ threefold $\mathcal{X}$ and for the topological A-model on a CY threefold $\hat{\mathcal{X}}$, provided one replaces the Euler number $\chi(\mathcal{X})$ by $-\chi(\hat{\mathcal{X}})$. Indeed, mirror symmetry $\mathcal{X} \mapsto \hat{\mathcal{X}}$ exchanges the two topological wave functions [45, 71]. We shall now proceed to discuss some key features of the A-model, which will play an important role in section 5 .

In contrast to the B-model, the A-model topological string encodes deformations of the (complexified) Kähler structure of the mirror Calabi-Yau threefold $\hat{\mathcal{X}}$. The holomorphic wave function $F_{\text {hol }}\left(z^{a}, \lambda\right)$ of the topological A-model on $\hat{\mathcal{X}}$ therefore depends on the Kähler moduli $z^{a} \in \mathcal{M}_{K}(\hat{\mathcal{X}})$ together with the topological string coupling $\lambda$. The A-model wave function $F_{\text {hol }}\left(z^{a}, \lambda\right)$ is moreover related to the partition function of Gromov-Witten (GW) invariants via

$$
\exp \left[F_{\mathrm{hol}}\left(z^{a}, \lambda\right)-F_{\mathrm{pol}}\left(z^{a}, \lambda\right)\right]=Z_{\mathrm{GW}}
$$

where

$$
F_{\mathrm{pol}}\left(z^{a}, \lambda\right)=-\frac{(2 \pi \mathrm{i})^{3}}{\lambda^{2}}\left(\frac{1}{6} \kappa_{a b c} z^{a} z^{b} z^{c}-\frac{1}{2} A_{\Lambda \Sigma} z^{\Lambda} z^{\Sigma}\right)-\frac{2 \pi \mathrm{i}}{24} c_{2, a} z^{a}
$$

is the "polar part" of $F_{\text {hol }}\left(z^{a}, \lambda\right)$, and $\kappa_{a b c}$ is the intersection product on $H^{3}(\hat{\mathcal{X}}, \mathbb{Z})$. Here we included the "quadratic ambiguity" $A_{\Lambda \Sigma} z^{\Lambda} z^{\Sigma}$ in the prepotential, where $A_{\Lambda \Sigma}$ is a constant real symmetric matrix and $z^{\Lambda}=\left(1, z^{a}\right)$. This quadratic term does not affect the metric on the Kähler moduli space but plays a crucial role for charge quantization, as we demonstrate 
in section 4 . The right hand side of (2.78) involves the partition function $Z_{\mathrm{GW}}=Z_{\mathrm{GW}}^{0} Z_{\mathrm{GW}}^{\prime}$, where $Z_{\mathrm{GW}}^{\prime}$ encodes the non-degenerate GW-invariants, while

$$
Z_{\mathrm{GW}}^{0}=\lambda^{-\frac{\chi(\hat{\mathcal{X}})}{24} \epsilon_{\mathrm{GW}}}\left[M\left(e^{-\lambda}\right)\right]^{\chi(\hat{\mathcal{X}}) / 2}
$$

contains the contribution from degenerate GW-invariants. This follows from the weak coupling expansion of the Mac-Mahon function $M(q)=\prod\left(1-q^{n}\right)^{-n}$ (see e.g. appendix E in $[72])$,

$$
M\left(e^{-\lambda}\right) \sim K \lambda^{\frac{1}{12}} \exp \left(\frac{\zeta(3)}{\lambda^{2}}+\sum_{n=0}^{\infty}(-1)^{n} \lambda^{2 n+2} \frac{\left|B_{2 n+4}\right|}{(2 n+4) !} \frac{(2 n+3)}{(2 n+2)} B_{2 n+2}\right),
$$

where $B_{n}$ are the Bernoulli numbers and $K$ is a numerical constant. Upon choosing the parameter $\epsilon_{\mathrm{GW}}$ in (2.80) equal to one, the power of $\lambda$ in (2.80) cancels the similar power in (2.81), as usually assumed in the topological string theory literature. In $[72,73]$, it was however noticed that the choice $\epsilon_{\mathrm{GW}}=0$ was necessary in order to match the OSV conjecture. We do not take sides at this point and leave the parameter $\epsilon_{\mathrm{GW}}$ arbitrary.

We further note that $Z_{\mathrm{GW}}^{\prime}$ is related to the partition function of the (ordinary, rank one) Donaldson-Thomas invariants $N_{D T}\left(Q_{a}, 2 J\right)$ via the "GW/DT relation" [60,61]

$$
Z_{\mathrm{GW}}^{\prime}=\left[M\left(e^{-\lambda}\right)\right]^{-\chi(\hat{\mathcal{X}}) \epsilon_{\mathrm{DT}}} Z_{\mathrm{DT}},
$$

where the DT partition function is defined as

$$
Z_{\mathrm{DT}} \equiv \sum_{Q_{a}, J}(-1)^{2 J} N_{\mathrm{DT}}\left(Q_{a}, 2 J\right) e^{-2 \lambda J+2 \pi \mathrm{i} Q_{a} z^{a}} .
$$

In the relation (2.82) we have also allowed for an arbitrary parameter $\epsilon_{\mathrm{DT}}$, which is usually taken to be equal to one in the literature. We shall nevertheless leave it unspecified for now. Physically, the DT invariants count bound states of one D6-brane with $2 J$ D0-branes and $Q_{a}$ D2-branes wrapped along $\gamma^{a} \in H_{2}(\hat{\mathcal{X}}, \mathbb{Z})$. They are also expected to provide the instanton measure for D5-D1-D(-1) Euclidean configurations.

We may now combine the above relations and express the holomorphic wave function $e^{F_{\mathrm{hol}}(z, \lambda)}$ in terms of the DT partition function:

$$
e^{F_{\mathrm{hol}}(z, \lambda)}=\lambda^{-\frac{\chi(\hat{\mathcal{X}})}{24} \epsilon_{\mathrm{GW}}}\left[M\left(e^{-\lambda}\right)\right]^{\left(\frac{1}{2}-\epsilon_{\mathrm{DT}}\right) \chi(\hat{\mathcal{X}})} e^{F_{\mathrm{pol}}} \sum_{Q_{a}, J}(-1)^{2 J} N_{\mathrm{DT}}\left(Q_{a}, 2 J\right) e^{-2 \lambda J+2 \pi \mathrm{i} Q_{a} z^{a}} .
$$

This relation will play an important role in section 5.4.

\section{Topology of the type IIA hypermultiplet moduli space}

We shall now discuss quantum corrections to the hypermultiplet (HM) moduli space $\mathcal{M}=$ $\mathcal{Q}_{c}(\mathcal{X})$ in type IIA string theory compactified on a CY 3 -fold $\mathcal{X}$. By the usual T-duality and mirror symmetry arguments reviewed e.g. in $[18,54]$, the same space $\mathcal{M}$ also appears as the HM moduli space in M-theory on $\mathcal{X}$, as the HM moduli space in type IIB on the mirror 


\begin{tabular}{|c|c|c|c|c|}
\hline & \multicolumn{2}{|c|}{ IIA $/ \mathcal{X} \times S_{R}^{1}$} & \multicolumn{2}{c|}{$\mathrm{IIB} / \hat{\mathcal{X}} \times S_{R^{\prime}}^{1}$} \\
\hline & HM & VM & VM & HM \\
\hline $\mathcal{M}$ & $\mathcal{Q}_{c}(\mathcal{X})$ & $\mathcal{Q}_{K}(\mathcal{X})$ & $\mathcal{Q}_{c}(\hat{\mathcal{X}})$ & $\mathcal{Q}_{K}(\hat{\mathcal{X}})$ \\
$r$ & $1 / g_{(4)}^{2}$ & $\left(R / l_{(4)}\right)^{2}$ & $\left(R / l_{(4)}\right)^{2}$ & $1 / g_{(4)}^{2}$ \\
$\mathcal{O}\left(e^{-\sqrt{r}}\right)$ & $\mathrm{D} 2$ & $\mathrm{D} 0-\mathrm{D} 2-\mathrm{D} 4-\mathrm{D} 6$ & $\mathrm{D} 3$ & $\mathrm{D}(-1)-\mathrm{D} 1-\mathrm{D} 3-\mathrm{D} 5$ \\
$\mathcal{O}\left(e^{-r}\right)$ & $\mathrm{NS} 5$ & $\mathrm{KKM}$ & $\mathrm{KKM}$ & $\mathrm{NS} 5$ \\
\hline
\end{tabular}

Table 1. Dictionary between various realizations of $\mathcal{Q}_{c}(\mathcal{X})=\mathcal{Q}_{K}(\hat{\mathcal{X}})$.

3-fold $\hat{\mathcal{X}}$, as the VM moduli space in type IIA on $\hat{\mathcal{X}} \times S^{1}$ or as the VM moduli space in type IIB theories on $\mathcal{X} \times S^{1}$ (see table 1 ). Here using insights from the previous section, we clarify the qualitative structure of NS5-instanton corrections to the hypermultiplet metric, a result which allows us to specify the topology of the perturbative moduli space in the weak coupling limit. Some of the results in this section were already announced in [1].

\subsection{Perturbative HM moduli space}

The HM moduli space in type IIA string theory compactified on a CY 3 -fold $\mathcal{X}$ is a quaternion-Kähler manifold $\mathcal{M}=\mathcal{Q}_{c}(\mathcal{X})$ of real dimension $2 b_{3}(\mathcal{X})=4\left(h_{2,1}+1\right)$. Here the subscript $c$ refers to the fact that $\mathcal{Q}_{c}(\mathcal{X})$ encodes the moduli space of complex structures $\mathcal{M}_{c}(\mathcal{X})$, as well as the four-dimensional dilaton $r \equiv e^{\phi} \sim 1 / g_{(4)}^{2}$, RR-field $C$ and NS axion $\sigma$. In the weak coupling limit $r \rightarrow \infty$, the quaternion-Kähler metric on $\mathcal{M}$ is given, to all orders in $1 / r$, by $[14,16,17]$

$d s_{\mathcal{M}}^{2}=\frac{r+2 c}{r^{2}(r+c)} \mathrm{d} r^{2}+\frac{4(r+c)}{r} \mathrm{~d} s_{\mathcal{S} \mathcal{K}}^{2}+\frac{\mathrm{d} s_{\mathcal{T}}^{2}}{r}+\frac{2 c}{r^{2}} e^{\mathcal{K}}\left|z^{\Lambda} \mathrm{d} \tilde{\zeta}_{\Lambda}-F_{\Lambda} \mathrm{d} \zeta^{\Lambda}\right|^{2}+\frac{r+c}{16 r^{2}(r+2 c)} D \sigma^{2}$.

Here, $\left(\zeta^{\Lambda}, \tilde{\zeta}_{\Lambda}\right)$ are the real periods $(2.6)$ of the $C$-field on a symplectic basis of $H^{3}(\mathcal{X}, \mathbb{R}), \mathrm{d} s_{\mathcal{I}}^{2}$ is the metric (2.10) on the (torus fiber of the) intermediate Jacobian, $\mathrm{d} s_{\mathcal{S K}}^{2}=\mathcal{K}_{a \bar{b}} \mathrm{~d} z^{a} \mathrm{~d} \bar{z}^{\bar{b}}$ is the special Kähler metric on $\mathcal{S K} \equiv \mathcal{M}_{c}(\mathcal{X})$, with Kähler connection (2.4), $D \sigma$ is the one-form

$$
D \sigma=\mathrm{d} \sigma+\tilde{\zeta}_{\Lambda} \mathrm{d} \zeta^{\Lambda}-\zeta^{\Lambda} \mathrm{d} \tilde{\zeta}_{\Lambda}+8 c \mathcal{A}_{K}
$$

and $c$ is a deformation parameter which encodes the one-loop correction,

$$
c=-\chi(\mathcal{X}) /(192 \pi) .
$$

In the special case where $\chi(\mathcal{X})=0$ (or to leading, tree-level approximation in the weak coupling limit $r \rightarrow \infty$ ), the metric (3.1) follows from the special Kähler metric on $\mathcal{S} \mathcal{K}$ by the $c$-map construction [6, 7]. All higher loop corrections are presumed to vanish, for reasons that will be recalled shortly. On the other hand, non-perturbative corrections from D2brane and NS5-brane instantons will correct (3.1) at order $e^{-\sqrt{r}}$ and $e^{-r}$, respectively [3]. In particular, such corrections should resolve the curvature singularity of the perturbative metric (3.1) which is present at $r=-2 c$ when $\chi(\mathcal{X})>0$. 


\subsubsection{Ten-dimensional origin of the one-loop correction}

It is interesting to note that the connection term in (3.2) amounts, after dualizing the NS-axion $\sigma$ and the RR-axions $\tilde{\zeta}_{\Lambda}$ into two-form potentials $B$ and $B^{\Lambda}$, to a topological coupling

$$
\int\left(\operatorname{Re} \mathcal{N}_{\Lambda \Sigma}\left(\mathrm{d} B^{\Lambda}+\zeta^{\Lambda} \mathrm{d} B\right) \wedge d \zeta^{\Sigma}-8 c B \wedge \omega_{\mathcal{S K}}\right)
$$

in the $4 \mathrm{D}$ effective action. ${ }^{18}$ This in turn follows by dimensional reduction of the topological coupling in 10-dimensional type IIA supergravity

$$
\int_{\mathcal{Y}}\left(\frac{1}{6} B \wedge \mathrm{d} C \wedge \mathrm{d} C-B \wedge I_{8}(R)\right),
$$

where $I_{8}(R)=\left(p_{2}-\frac{1}{4} p_{1}^{2}\right) / 48$ on $\mathcal{X}$ (or from the similar coupling in $11 \mathrm{D}$ supergravity on $\left.\mathcal{X} \times S^{1}\right)$. Indeed, using the standard relations between Pontryagin classes $p_{1}=c_{1}^{2}-2 c_{2}$ and $p_{2}=c_{2}^{2}-2 c_{1} c_{3}+2 c_{4}$ (see e.g. [74], section 6.4), the second term in (3.5) can be rewritten, on an arbitrary complex manifold $\mathcal{Y}$, as

$$
B \wedge I_{8}=\frac{1}{24} B \wedge\left[c_{4}-c_{1}\left(c_{3}+\frac{1}{8} c_{1}^{3}-\frac{1}{2} c_{1} c_{2}\right)\right] .
$$

Taking ${ }^{19} \mathcal{Y}=\mathbb{R}^{4} \times \mathcal{X}$, where the complex structure of $\mathcal{X}$ varies as a function of the position in $\mathbb{R}^{4}$, and integrating the term in parenthesis on the $\mathrm{CY}$ threefold $\mathcal{X}$ produces a coupling $-\frac{\chi(\mathcal{X})}{24} B \wedge c_{1}$ on $\mathbb{R}^{4}$, which by the arguments in [45] is equal to $\frac{\chi(\mathcal{X})}{24} B \wedge \omega_{\mathcal{S K}}$. This derivation of the one-loop correction to the metric can be viewed as a supersymmetric counterpart of the derivation in $[13,15],{ }^{20}$ where corrections to the scale factors in the metric (3.1) were obtained by reduction of the CP-even $R^{4}$-type couplings in 10 dimensional type IIA supergravity.

\subsection{Topology and instanton corrections}

While (3.1) describes the local, Riemannian geometry of $\mathcal{M}$, it is of prime importance to understand its topology. Since the metric (3.1) is only valid in the weak coupling regime, we shall restrict our discussion to the topology of the hypersurfaces $\mathcal{C}(r)$, corresponding to fixed values of the dilaton $r \in \mathbb{R}_{+}$, which foliate the full moduli space $\mathcal{M}$. The global structure of $\mathcal{C}(r)$ (which, evidently, must be independent of $r$ ) can be specified by providing a group of discrete identifications of the coordinates $z^{a}, \zeta^{\Lambda}, \tilde{\zeta}_{\Lambda}, \sigma$, acting isometrically on $\mathcal{C}(r)$. The precise subgroup can be identified by studying the allowed instanton corrections to the metric. In the rest of this section, we shall discuss instanton corrections to (3.1) at a qualitative, semi-classical level, ignoring the tensorial nature of the metric. A more precise discussion will be given in section 5 using twistor techniques.

\footnotetext{
${ }^{18}$ To dualize the NS-axion, we add a term $-\mathrm{d} B \wedge \frac{\mathrm{d} \sigma}{2}$ to the Lagrangian $\mathrm{d} s_{\mathcal{M}}^{2}$ and integrate out the one-form $\mathrm{d} \sigma$, leading to $-\frac{1}{6} r^{2} \mathrm{~d} B \wedge * \mathrm{~d} B+B \wedge\left(\omega_{\mathcal{T}}+\frac{\chi(\mathcal{X})}{24} \omega_{\mathcal{S K}}\right)$, where $\omega_{\mathcal{S K}}=-\frac{1}{2 \pi} \mathrm{d} \mathcal{A}_{K}$.

${ }^{19}$ Taking instead $\mathcal{Y}=\mathbb{R}^{2} \times \mathcal{G}$, where $\mathcal{G}$ is a $\mathrm{CY}$ four-fold, eq. (3.6) reproduces the $\frac{1}{24} \chi_{\mathcal{G}} B$ one-point function found in [75]. When $\mathcal{G}$ is a $\mathrm{CY}$ threefold fibered over $\mathbb{C} P^{1},(3.6)$ should instead reduce to Eq (19) in $[76]$.

${ }^{20}$ Note that the analysis of [15] was restricted to the "universal sector", where the $B \wedge \omega_{\mathcal{S K}}$ coupling vanishes. In particular, for rigid CY threefolds, the last term in (3.2) is absent. We are grateful to R. Minasian and P. Vanhove for discussions on these issues.
} 


\subsubsection{Instanton contributions to the HM moduli space}

In type IIA string theory on $\mathcal{X}$, these correspond to D2-brane instantons wrapping a sLag submanifold of $\mathcal{X}$ (more precisely, to stable objects in the Fukaya category of $\mathcal{X}$ ). Such instantons produce corrections to the metric on $\mathcal{Q}_{c}(\mathcal{X})$ of the form [18]

$$
\left.\delta \mathrm{d} s^{2}\right|_{\mathrm{D} 2} \sim \exp \left(-8 \pi e^{\phi / 2}\left|Z_{\gamma}\right|-2 \pi \mathrm{i}\left(q_{\Lambda} \zeta^{\Lambda}-p^{\Lambda} \tilde{\zeta}_{\Lambda}\right)\right)
$$

where $\left(p^{\Lambda}, q_{\Lambda}\right)$ are integers which label the homology class $\gamma=q_{\Lambda} \mathcal{A}^{\Lambda}-p^{\Lambda} \mathcal{B}_{\Lambda} \in H_{3}(\mathcal{X}, \mathbb{Z})$ of the sLag, while the central charge $Z_{\gamma} \equiv e^{\mathcal{K} / 2}\left(q_{\Lambda} X^{\Lambda}-p^{\Lambda} F_{\Lambda}\right)$ (or, in mathematical parlance, the stability data) is the volume of the sLag, induced from the CY metric. The expression (3.7) is only valid in the classical, weak coupling limit $r \rightarrow \infty$, up to a one-loop determinant prefactor which we discuss in section 3.2.3. There are also multi-D-instanton corrections to the metric, but the dependence on the axions $(\zeta, \tilde{\zeta}, \sigma)$ is always of the form (3.7), where $\left(p^{\Lambda}, q_{\Lambda}\right)$ is the total charge carried by the multi-instanton configuration.

At subleading order $e^{-r}$, there are in addition corrections from NS5-brane instantons in the homology class $k \mathcal{X}$ [3]. For $k>0$, these corrections are expected to be of the form

$$
\left.\delta \mathrm{d} s^{2}\right|_{\mathrm{NS} 5} \sim e^{-4 \pi k e^{\phi}-\mathrm{i} \pi k \sigma} \mathcal{Z}_{\mathrm{NS} 5-\mathrm{G}}^{(k)}\left(\mathcal{N}, \zeta^{\Lambda}, \tilde{\zeta}_{\Lambda}\right),
$$

where $\mathcal{Z}_{\mathrm{NS} 5-\mathrm{G}}^{(k)}$ is the NS5-partition function in the Gaussian, weak coupling approximation, while the prefactor in (3.8) incorporates the Euclidean action for $k$ NS5-branes. For the purposes of reading off from (3.8) the classical NS5-brane action, one can replace $\mathcal{Z}_{\text {NS5-G }}^{(k)}$ with the self-dual flux partition function $\mathcal{Z}_{\Theta, \mu}^{(k)}$ obtained in (2.35). Then retaining one of the terms in the sum (2.35) and disregarding normalization factors, the classical action for the non-perturbative corrections in (3.8) is found to be

$$
\begin{aligned}
S_{\mathrm{NS} 5 / \mathrm{D} 2}= & \pi k\left[4 e^{\phi}-\mathrm{i}\left(n^{\Lambda}-\zeta^{\Lambda}\right) \overline{\mathcal{N}}_{\Lambda \Sigma}\left(n^{\Sigma}-\zeta^{\Sigma}\right)\right] \\
& +\mathrm{i} \pi k\left(\sigma+\zeta^{\Lambda} \tilde{\zeta}_{\Lambda}\right)-2 \pi \mathrm{i} k\left(\tilde{\zeta}_{\Lambda}-\phi_{\Lambda}\right) n^{\Lambda}-\mathrm{i} \pi k \theta^{\Lambda} \phi_{\Lambda} .
\end{aligned}
$$

The first line matches precisely the instanton action obtained in [49], eq. (4.54), based on a classical analysis of instanton solutions in $\mathcal{N}=2$ supergravity, while the second line restores the appropriate axionic couplings (including characteristics). The integers $k n^{\Lambda}$ may be interpreted as the flux on the fivebrane, or as charges of a D2-brane wrapped on $k n^{\Lambda} \mathcal{B}_{\Lambda} \in H_{3}(\mathcal{X}, \mathbb{Z})$, although this classical interpretation is misleading since fluxes or D2-brane charges are inherently quantum-mechanical on the fivebrane worldvolume.

\subsubsection{Large gauge transformations}

The isometry group of $\mathcal{C}(r)$ contains in particular the continuous translations

$$
T_{H, \kappa}: \quad\left(\zeta^{\Lambda}, \tilde{\zeta}_{\Lambda}, \sigma\right) \mapsto\left(\zeta^{\Lambda}+\eta^{\Lambda}, \tilde{\zeta}_{\Lambda}+\tilde{\eta}_{\Lambda}, \sigma+2 \kappa-\tilde{\eta}_{\Lambda} \zeta^{\Lambda}+\eta^{\Lambda} \tilde{\zeta}_{\Lambda}\right),
$$

with $H=\left(\eta^{\Lambda}, \tilde{\eta}_{\Lambda}\right) \in \mathbb{R}^{b_{3}}, \kappa \in \mathbb{R}$, satisfying the Heisenberg group law

$$
T_{H_{2}, \kappa_{2}} T_{H_{1}, \kappa_{1}}=T_{H_{1}+H_{2}, \kappa_{1}+\kappa_{2}+\frac{1}{2}\left\langle H_{1}, H_{2}\right\rangle},
$$


where $\left\langle H, H^{\prime}\right\rangle$ is the symplectic pairing (2.22). While being a symmetry to all orders in $1 / r$, the continuous shifts $T_{H, 0}$ are generally expected to be broken to a discrete subgroup by D-instantons. The form of the D-instanton corrections (3.7) implies that $\partial_{\sigma}$ continues to be an isometry of the HM metric at order $e^{-\sqrt{r}}$, but that invariance under continuous translations of the $C$-field is broken to the discrete group $\Gamma=H^{3}(\mathcal{X}, \mathbb{Z})$, dual to the lattice $H_{3}(\mathcal{X}, \mathbb{Z})$ of D2-brane charges, acting by integer shifts on the $\mathrm{RR}$ axions

$$
\left(\zeta^{\Lambda}, \tilde{\zeta}_{\Lambda}\right) \mapsto\left(\zeta^{\Lambda}+\eta^{\Lambda}, \tilde{\zeta}_{\Lambda}+\tilde{\eta}_{\Lambda}\right)
$$

where $H=\left(\eta^{\Lambda}, \tilde{\eta}_{\Lambda}\right) \in H^{3}(\mathcal{X}, \mathbb{Z})$. In order to be an isometry of the perturbative metric (3.1), this must be accompanied by a shift of the NS-axion, so that $\Gamma$ acts as the transformation $T_{H, 0}$ in (3.10), possibly combined with a certain translation $T_{0, c(H)}$ of $\sigma$ which, at this order, remains undetermined. Thus, ignoring the NS-axion $\sigma$ and keeping the complex structure of $\mathcal{X}$ fixed, the hypersurface $\mathcal{C}(r)$ projects to the intermediate Jacobian torus $\mathcal{T}=H^{3}(\mathcal{X}, \mathbb{R}) / H^{3}(\mathcal{X}, \mathbb{Z})$, as stated in [8].

The NS5-brane corrections (3.8) further break the continuous translations in $\sigma$ to discrete identifications ${ }^{21} T_{(0, \kappa)}: \sigma \mapsto \sigma+2 \kappa, \kappa \in \mathbb{Z}$. This implies that the hypersurface $\mathcal{C}(r)$ is a circle bundle over the intermediate Jacobian $\mathcal{J}_{c}(\mathcal{X})$, the fiber of which is parametrized by $e^{\mathrm{i} \pi \sigma}, 0 \leq \sigma<2$. In addition, eq. (3.8) implies that the restriction of $\mathcal{C}(r)$ to the torus $\mathcal{T}$ must be isomorphic to the circle bundle $\mathcal{C}_{\Theta}$ where the NS5-brane partition function is valued. In particular, the shift $T_{0, c(H)}$ which must accompany the large gauge transformation (3.12) follows from the transformation property (2.36) of the fivebrane partition function. This shift is given by

$$
c(H)=-\frac{1}{2} \eta^{\Lambda} \tilde{\eta}_{\Lambda}+\tilde{\eta}_{\Lambda} \theta^{\Lambda}-\eta^{\Lambda} \phi_{\Lambda} \bmod 1
$$

where $\Theta=\left(\theta^{\Lambda}, \phi_{\Lambda}\right)$ are the characteristics governing the chiral partition function (2.35). In particular, $c(H)$ satisfies

$$
c\left(H_{1}+H_{2}\right)=c\left(H_{1}\right)+c\left(H_{2}\right)+\frac{1}{2}\left\langle H_{1}, H_{2}\right\rangle \bmod 1,
$$

as a result of the quadratic refinement law $(2.21)$ for $\sigma_{\Theta}(H)=(-1)^{2 c(H)}$. Thus, large gauge transformations imply that the axions $(C, \sigma)$ take values in the quotient $\left(H^{3}(\mathcal{X}, \mathbb{Z}) \times \mathbb{R}\right) / \Gamma^{\prime}$, where $\Gamma^{\prime}$ is the group generated by $T_{H, \kappa}^{\prime} \equiv T_{H, \kappa+c(H)}$ acting via

$$
T_{H, \kappa}^{\prime}:\left(\zeta^{\Lambda}, \tilde{\zeta}_{\Lambda}, \sigma\right) \mapsto\left(\zeta^{\Lambda}+\eta^{\Lambda}, \tilde{\zeta}_{\Lambda}+\tilde{\eta}_{\Lambda}, \sigma+2 \kappa-\tilde{\eta}_{\Lambda} \zeta^{\Lambda}+\eta^{\Lambda} \tilde{\zeta}_{\Lambda}+2 c(H)\right),
$$

where $H=\left(\eta^{\Lambda}, \tilde{\eta}_{\Lambda}\right) \in H^{3}(\mathcal{X}, \mathbb{Z}), \kappa \in \mathbb{Z}$ and $c(H) \in \mathbb{R}$ is chosen such that (3.13) holds. It should be noted that the extra shift ${ }^{22}$ of $c(H)$ is crucial for the consistency of this action.

\footnotetext{
${ }^{21}$ Here we assume that $k$ is integer. This can be argued by carefully dualizing $\sigma$ as in footnote 18 , and will be confirmed shortly using S-duality.

${ }^{22}$ The shift in $\sigma$ induced by the quadratic refinement was already observed in the context of rigid CalabiYau compactifications upon assuming that the moduli space should be invariant under a certain natural arithmetic group [53]. With hindsight, this additional shift could also have been uncovered in the $\operatorname{SL}(3, \mathbb{Z})$ invariant construction of [54], had it not been obscured by a redefinition of $\kappa$.
} 
Indeed, the composition of two large gauge transformations

$$
T_{H_{2}, \kappa_{2}}^{\prime} T_{H_{1}, \kappa_{1}}^{\prime}=T_{H_{1}+H_{2}, \kappa_{1}+\kappa_{2}+c\left(H_{1}\right)+c\left(H_{2}\right)+\frac{1}{2}\left\langle H_{1}, H_{2}\right\rangle-c\left(H_{1}+H_{2}\right)}^{\prime}
$$

is again a large gauge transformation $T_{H_{3}, \kappa_{3}}^{\prime}$ with $\kappa_{3} \in \mathbb{Z}$, by virtue of (3.14). Thus, upon acting on functions of the form $F_{k}\left(\zeta^{\Lambda}, \tilde{\zeta}_{\Lambda}, \sigma\right) \equiv F_{k}\left(\zeta^{\Lambda}, \tilde{\zeta}_{\Lambda}\right) e^{\mathrm{i} \pi k \sigma}$ with $k$ integer, large gauge transformations $T_{H, \kappa}^{\prime}$ are effectively Abelian.

To summarize, at a fixed point in complex structure moduli space we have found that the circle bundle $\mathcal{C}$ indeed restricts to the circle bundle $\mathcal{C}_{\Theta}$ over the torus $\mathcal{T}=$ $H^{3}(\mathcal{X}, \mathbb{R}) / H^{3}(\mathcal{X}, \mathbb{Z})$ governing the fivebrane partition function. We now discuss the fibration of $\mathcal{C}(r)$ over the complex structure moduli space.

\subsubsection{Monodromies}

For discussing the fibration of the circle bundle $\mathcal{C}(r)$ over $\mathcal{M}_{c}(\mathcal{X})$, and in particular its behavior under monodromies, it becomes important to include the normalization factors for the D-instanton and NS5-instanton corrections (3.7) and (3.8).

In the case of D-instantons, the normalization factor was determined in [18, 24]. While the answer depends on the metric component under consideration (see section 5.2.2 for details), the normalization factor universally involves the product $\Omega(\gamma) \sigma_{\mathrm{D}}(\gamma)$, where $\Omega(\gamma)$ is the generalized DT invariant associated to the sLag submanifold $\gamma=\left(p^{\Lambda}, q_{\Lambda}\right)$, and $\sigma_{\mathrm{D}}(\gamma)$ is again a quadratic refinement ${ }^{23}$ of the intersection form on $H^{3}(\mathcal{X}, \mathbb{Z})$. As in the case of the fivebrane partition function, $\sigma_{\mathrm{D}}(\gamma)$ may be parametrized by characteristics $\Theta_{\mathrm{D}}$,

$$
\sigma_{\mathrm{D}}(\gamma)=\mathbf{E}\left(-\frac{1}{2} q_{\Lambda} p^{\Lambda}+q_{\Lambda} \theta_{\mathrm{D}}^{\Lambda}-p^{\Lambda} \phi_{\mathrm{D}, \Lambda}\right) \equiv \sigma_{\Theta_{\mathrm{D}}}(\gamma)
$$

In order that the product $\Omega(\gamma) \sigma_{\mathrm{D}}(\gamma)$ be invariant, $\Theta_{D}$ must transform in the same way (2.24) as the fivebrane characteristics $\Theta$ under monodromies $C \mapsto \rho(M) \cdot C$.

While it seems natural to identify the two set of characteristics, $\Theta$ and $\Theta_{D}$, we do not know for sure that this is a consistent choice. On the one hand, it is desirable that physics (in particular the HM moduli space $\mathcal{M}$ ) be independent on any choice of quadratic refinement, as it is the case for the class of $\mathcal{N}=2$ field theories considered in [78]. For what concerns the D-instanton corrections (3.7), a change $\Theta_{\mathrm{D}} \mapsto \Theta_{\mathrm{D}}^{\prime}$ of the D-instanton characteristics can be canceled by redefining the coordinate $C=(\zeta, \tilde{\zeta})$ into $^{24}$

$$
C^{\prime}=C+\Theta_{\mathrm{D}}^{\prime}-\Theta_{\mathrm{D}}
$$

Similarly, for what concerns the NS5-instanton corrections (3.8), a change $\Theta \mapsto \Theta^{\prime}$ of the fivebrane characteristics can be canceled by redefining

$$
\hat{C}=C+\Theta^{\prime}-\Theta, \quad \hat{\sigma}=\sigma+\left\langle\Theta-\Theta^{\prime}, C\right\rangle-\left\langle\Theta, \Theta^{\prime}\right\rangle,
$$

\footnotetext{
${ }^{23}$ It would be very interesting to obtain $\sigma_{\mathrm{D}}(\gamma)$ from the one-loop determinant for the topological field theory on the D2-brane, along the lines of the superpotential computation in [77].

${ }^{24}$ The cost to pay is that $C^{\prime}$ no longer transforms as a symplectic vector under monodromies, as observed in section 4.1 of [24].
} 
where we have used (2.37). In order for these two field redefinitions to be consistent, we require that $\Theta_{D}$ and $\Theta$ should vary in the same way. This suggests that the difference $\Theta-\Theta_{D}$ should be fixed, although it does not yet imply that it should vanish. Now, it follows from (2.24) that the difference of characteristics transforms as a symplectic vector under monodromies, modulo the addition of integers. In general, there is no non-zero vector invariant under the full monodromy group, and so no canonical choice for the difference $\Theta-\Theta_{D}$ except zero. On the other hand, while type IIB S-duality suggests that the two quadratic refinements should be related (as one governs NS5-brane instantons while the other controls D5-brane instantons) in section 5.3 we shall find some tension between the equality of the two characteristics and S-duality. In view of this, we shall continue to distinguish the two quadratic refinements in the sequel.

We now turn to the monodromy invariance of the fivebrane instanton correction (3.8), and to the topology of the NS-axion circle bundle $\mathcal{C}$ over the complex structure moduli space $\mathcal{M}_{c}(\mathcal{X})$. As a first approach to this problem, let us evaluate the curvature $\mathrm{d}(D \sigma / 2)$ of the horizontal one-form (3.2), suitably normalized to take into account the mod 2 periodicity of $\sigma$ :

$$
\mathrm{d}\left(\frac{D \sigma}{2}\right)=\omega_{\mathcal{T}}+\frac{\chi(\mathcal{X})}{24} \omega_{\mathcal{S K}}
$$

The first term, equal to the Kähler class on the intermediate Jacobian torus $\mathcal{T}$, is recognized as the curvature of the circle bundle $\mathcal{C}_{\Theta}$ discussed in section 2.3. The second term, proportional to the Kähler class of the complex structure moduli space $\mathcal{M}_{c}(\mathcal{X})$, suggests that the restriction of $\mathcal{C}$ to $\mathcal{M}_{c}(\mathcal{X})$ is isomorphic to $\mathcal{L}^{\frac{\chi(\mathcal{X})}{24}}$, where $\mathcal{L}$ is the Hodge line bundle over $\mathcal{M}_{c}(\mathcal{X})$. Unless $\chi(\mathcal{X})$ is divisible by 24 , there is no canonical definition of $\mathcal{L}^{\frac{\chi(\mathcal{X})}{24}}$ (equivalently, the curvature (3.20) is not an integer cohomology class). This suggests that $\mathcal{C}$ is a twisted circle bundle and that the coordinate $\sigma$ is in fact not globally well-defined, as already observed in a slightly different context in [79].

To address this point in more detail, observe that under local holomorphic rescalings $\Omega_{3,0} \mapsto e^{f} \Omega_{3,0}$, the requirement that the horizontal one-form (3.2) be invariant implies that $\sigma$ must shift according to

$$
\sigma \mapsto \sigma+\frac{\chi(\mathcal{X})}{24 \pi} \operatorname{Im} f+2 \kappa_{f}
$$

(indeed, recall from $(2.5)$ that $\mathcal{A}_{K} \mapsto \mathcal{A}_{K}+\mathrm{d} \operatorname{Im} f$ under Kähler transformations $\mathcal{K} \mapsto$ $\mathcal{K}-f-\bar{f})$. Here, $\kappa_{f}$ is an undetermined constant modulo 1 . More generally, under a monodromy transformation $M$ in $\mathcal{M}_{c}(\mathcal{X}), C$ and $\sigma$ must transform as

$$
C \mapsto \rho(M) \cdot C, \quad \sigma \mapsto \sigma+\frac{\chi(\mathcal{X})}{24 \pi} \operatorname{Im} f_{M}+2 \kappa(M),
$$

where $f_{M}$ is a local holomorphic function on $\mathcal{M}_{c}(\mathcal{X})$ determined by the rescaling $\Omega_{3,0} \mapsto$ $e^{f_{M}} \Omega_{3,0}$ of the holomorphic 3 -form around the monodromy, and $\kappa(M)$ is again an undetermined constant defined modulo 1 . The consistency of the monodromy transformations (3.22) requires that $e^{2 \pi \mathrm{i} \kappa(M)}$ be a unitary character of the monodromy group. The combination $s \equiv e^{-\frac{\chi(\mathcal{X})}{48} \mathcal{K}+\mathrm{i} \pi \sigma}$ then transforms as

$$
s \mapsto e^{2 \pi \mathrm{i} \kappa(M)} e^{\frac{\chi}{24} f} s,
$$


which may be taken as the definition of the twisted bundle $\mathcal{L}^{\frac{\chi(\mathcal{X})}{24}}$. Since the bundle $\mathcal{L}$ itself is well-defined, the first factor must be a $\chi(\mathcal{X}) / 24$ :th root of unity, $\kappa(M) \in \chi(\mathcal{X}) \mathbb{Z} / 24$. Similarly, the additional term $2 \kappa_{f} \in \chi(\mathcal{X}) \mathbb{Z} / 12$ in (3.21) is needed to escape the paradox raised in [1]: in the absence of this term, a trivial rescaling $\Omega_{3,0} \mapsto e^{2 \pi \mathrm{i}} \Omega_{3,0}$ would lead to identifications $\sigma \equiv \sigma+\chi(\mathcal{X}) / 12$ in conflict with the periodicity modulo 2 of $\sigma$. Let us further recall that the twisted bundle $\mathcal{L}^{\frac{\chi(\mathcal{X})}{24}}$ from section 2.5 also arises in the B-model topological wave function, which is valued in $\mathcal{L}^{\frac{\chi(\mathcal{X})}{24}-1}[45]$.

In order to fully specify the topology of the circle bundle $\mathcal{C}$, it is necessary to determine the unitary character $e^{2 \pi \mathrm{i} \kappa(M)}$, and more to the point, its logarithm $\kappa(M)$. To appreciate the nature of this question, it is useful to recall a similar problem which arises in the study of classical modular forms: ${ }^{25}$ determine the modular properties of the logarithm of the Dedekind $\eta$ function. The answer to this question is well-known since the work of Dedekind (whose sums we shall encounter again in section 4.3), but is most satisfactorily understood in the language of determinant line bundles [80]. Transposing to the present context, we expect that the role of the Dedekind $\eta$ function will be played by the one-loop B-model topological amplitude $e^{f_{1}} / \sqrt{J_{\mathrm{G}}}$, and that $\kappa(M)$ should be computable from the fivebrane determinant line bundle along the lines of $[35,36,81]$. Indeed, we shall find in section 5.5 that the metric-dependent normalization factor of the fivebrane partition function in the weak coupling limit is proportional to the one-loop B-model topological amplitude, consistently with the coupling (1.2).

Summarizing, we have found that the HM moduli space $\mathcal{M}$, in the weak coupling limit, is foliated by a family of hypersurfaces $\mathcal{C}(r)$ which are circle bundles ${ }^{26}$ over the intermediate Jacobian (2.9),

$$
\mathcal{M} \sim \mathbb{R}_{r}^{+} \times\left(\begin{array}{ccc}
S_{\sigma}^{1} \rightarrow & \mathcal{C}(r) \\
& \downarrow \\
& \mathcal{J}_{c}(\mathcal{X})
\end{array}\right)
$$

whose curvature is given by (3.20). The perturbative moduli space may be defined globally by the metric (3.1), subject to identifications (3.22) under monodromies, (3.15) under large gauge transformations, and to possibly other identifications relating different leaves $\mathcal{C}(r)$, as required e.g. by S-duality.

\section{Mirror symmetry, S-duality and NS5-branes in type IIB}

So far we have worked exclusively within the type IIA picture. In this section we discuss the mirror map to type IIB where S-duality provides a powerful tool to analyze NS5-brane effects. We recall that mirror symmetry identifies the HM moduli space $\mathcal{Q}_{c}(\mathcal{X})$ in type IIA string theory compactified on a CY 3 -fold $\mathcal{X}$ with the HM moduli space $\mathcal{Q}_{K}(\hat{\mathcal{X}})$ in type IIB compactified on the mirror CY 3 -fold $\hat{\mathcal{X}}$. While the former extends the moduli space of complex structures $\mathcal{M}_{c}(\mathcal{X})$ with data about the dilaton, RR-fields and NS axion, the latter

\footnotetext{
${ }^{25}$ We are grateful to A. Neitzke and D. Zagier for suggesting this point of view.

${ }^{26}$ The dilaton factor $\mathbb{R}_{r}^{+}$may be used to formally convert this circle bundle into a $\mathbb{C}^{\times}$-bundle [82], although the total space $\mathcal{M}$ is not expected to carry a global complex structure.
} 
instead extends the moduli space of (complexified) Kähler structures $\mathcal{M}_{K}(\hat{\mathcal{X}})$ with similar data. In contrast to $\mathcal{M}_{c}(\mathcal{X})$, the special Kähler metric on $\mathcal{M}_{K}(\hat{\mathcal{X}})$ receives worldsheet instanton corrections, and is most easily obtained from $\mathcal{M}_{c}(\mathcal{X})$ by mirror symmetry.

- In section 4.1 we review the mirror-dual description of $\mathcal{M}=\mathcal{Q}_{c}(\mathcal{X})$ as the type IIB HM moduli space $\mathcal{M}=\mathcal{Q}_{K}(\hat{\mathcal{X}})$. In the process, we discuss charge quantization, and reconcile the apparent fractional charge shifts in the K-theory description of type IIB instantons (or type IIA black holes) with the manifestly integer charges $\gamma \in H_{3}(\mathcal{X}, \mathbb{Z})$ of type IIA instantons (or type IIB black holes). - In section 4.2, we then discuss the action of S-duality on the type IIB hypermultiplet moduli space in the large volume limit and incorporate a novel shift of the D3-brane axion $c_{a}$ needed to ensure consistency with charge quantization. - In section 4.3, we use this action to derive the classical action of $(p, k) 5$-branes. In particular, we show that this action is consistent with the Gaussian flux partition function discussed in section 2 in the weak coupling limit.

\subsection{Topology of the perturbative type IIB HM moduli space}

Recall that in the weak coupling limit, the metric on $\mathcal{M} \equiv \mathcal{Q}_{K}(\hat{\mathcal{X}})$ is again given by the one-loop corrected $c$-map metric (3.1), where the special Kähler manifold $\mathcal{S} \mathcal{K}=\mathcal{M}_{K}(\hat{\mathcal{X}})$ is now the moduli space of complexified Kähler structures, the torus $\mathcal{T}$ parametrizes the periods of the RR potential

$$
A^{\text {even }}=A^{(0)}+A^{(2)}+A^{(4)}+A^{(6)} \in H^{\text {even }}(\hat{\mathcal{X}}, \mathbb{R}),
$$

and the coordinates $r=e^{\phi} \sim 1 / g_{(4)}^{2}$ and $\sigma$ still parametrize the 4D string coupling and NS axion. The deformation parameter $c$ takes the same value $c=\chi_{\hat{\mathcal{X}}} / 192 \pi=-\chi(\mathcal{X}) / 192 \pi$ as in (3.3). We refer to the total space of the torus $\mathcal{T}$ over $\mathcal{M}_{K}(\hat{\mathcal{X}})$ as the symplectic Jacobian $\mathcal{J}_{K}(\hat{\mathcal{X}})$.

\subsubsection{Kähler moduli space}

To describe the geometry of $\mathcal{J}_{K}(\hat{\mathcal{X}})$ in more detail, let us choose a basis $\gamma^{a}, a=$ $1, \ldots, h^{1,1}(\hat{\mathcal{X}})$, of 2 -cycles (Poincaré dual to 4 -forms $\omega^{a}$ ), and a basis $\gamma_{a}$ of 4-cycles (Poincaré dual to 2 -forms $\omega_{a}$ ), such that

$$
\omega_{a} \wedge \omega_{b}=\kappa_{a b c} \omega^{c}, \quad \omega_{a} \wedge \omega^{b}=\delta_{a}^{b} \omega_{\hat{\mathcal{X}}}, \quad \int_{\gamma^{a}} \omega_{b}=\int_{\gamma_{b}} \omega^{a}=\delta_{b}^{a},
$$

where $\omega_{\hat{\mathcal{X}}}$ is the volume form, normalized to $\int_{\hat{\mathcal{X}}} \omega_{\hat{\mathcal{X}}}=1$, and $\kappa_{a b c}=\int_{\hat{\mathcal{X}}} \omega_{a} \omega_{b} \omega_{c}=\left\langle\gamma_{a}, \gamma_{b}, \gamma_{c}\right\rangle$ is the triple intersection product in $H_{4}(\hat{\mathcal{X}}, \mathbb{Z})$. The space $H^{\text {even }}(\mathcal{X})$ carries a symplectic form given by

$$
\left\langle H, H^{\prime}\right\rangle=\int_{\hat{\mathcal{X}}} H \wedge \tau\left(H^{\prime}\right),
$$

where $\tau$ flips the sign of the 2-form and 6-form part of $H^{\prime}$. It will be convenient to let $\omega^{\Lambda}=\left(\omega_{\hat{\mathcal{X}}}, \omega^{a}\right), \omega_{\Lambda}=\left(1, \omega_{a}\right)$ such that $\left(\omega^{\Lambda}, \omega_{\Lambda}\right)$ forms a symplectic basis of $H^{\text {even }}(\mathcal{X}, \mathbb{R})$.

The Kähler structure moduli space $\mathcal{M}_{K}(\hat{\mathcal{X}})$ may then be parametrized by the periods $z^{a} \equiv b^{a}+\mathrm{i} t^{a}=\int_{\gamma^{a}}(B+\mathrm{i} J)$ of the complexified Kähler form. In projective special coordinates 
$X^{\Lambda}=\left(X^{0}, X^{a}\right)$ such that $z^{a}=X^{a} / X^{0}$, the prepotential governing $\mathcal{M}_{K}(\hat{\mathcal{X}})$ is given by ${ }^{27}$ $F(X)=-\frac{N\left(X^{a}\right)}{X^{0}}+\frac{1}{2} A_{\Lambda \Sigma} X^{\Lambda} X^{\Sigma}+\chi(\hat{\mathcal{X}}) \frac{\zeta(3)\left(X^{0}\right)^{2}}{2(2 \pi \mathrm{i})^{3}}-\frac{\left(X^{0}\right)^{2}}{(2 \pi \mathrm{i})^{3}} \sum_{k_{a} \gamma^{a} \in H_{2}^{+}(\hat{\mathcal{X}})} n_{k_{a}}^{(0)} \operatorname{Li}_{3}\left[\mathbf{E}\left(k_{a} \frac{X^{a}}{X^{0}}\right)\right]$,

where $N\left(X^{a}\right) \equiv \frac{1}{6} \kappa_{a b c} X^{a} X^{b} X^{c}$, and $A_{\Lambda \Sigma}$ is a constant, real symmetric matrix. The matrix $A_{\Lambda \Sigma}$ is ambiguous up to integer shifts $A_{\Lambda \Sigma} \mapsto A_{\Lambda \Sigma}+\mathcal{B}_{\Lambda \Sigma}, \mathcal{B}_{\Lambda \Sigma} \in \mathbb{Z}$, and does not affect the Kähler potential $\mathcal{K}=-\log \left[\mathrm{i}\left(\bar{X}^{\Lambda} F_{\Lambda}-X^{\Lambda} \bar{F}_{\Lambda}\right)\right]$ on $\mathcal{M}_{\mathcal{K}}(\hat{\mathcal{X}})$. However, as we shall demonstrate shortly, it is crucial for charge quantization. In (4.4), the sum over effective divisors represents the effect of genus 0 worldsheet instantons, while the trilogarithm sum $\operatorname{Li}_{3}(z) \equiv \sum_{n=1}^{\infty} z^{n} / n^{3}$ encodes multi-covering effects.

\subsubsection{Charge quantization}

By the same reasoning as in the type IIA analysis of section 2.1, the torus $\mathcal{T}$ in the mirror type IIB can be written as

$$
\mathcal{T}=H^{\text {even }}(\hat{\mathcal{X}}, \mathbb{R}) / \Gamma
$$

where $\Gamma$ is the lattice dual to the charge lattice classifying D5-D3-D1-D(-1)-instanton configurations. To determine this lattice, we must invoke the K-theory description of D-brane charges [83].

For non-vanishing D5-brane charge $p^{0}$, D5-D3-D1-D(-1)-instanton configurations can be represented as a coherent sheaf $E$ on $\hat{\mathcal{X}}$, of $\operatorname{rank} \operatorname{rk}(E)=p^{0}$ and generalized Mukai vector $\gamma^{\prime}$, defined by ${ }^{28}[83]$

$$
\gamma^{\prime} \equiv \operatorname{ch}(E) \sqrt{\operatorname{Td}(\hat{\mathcal{X}})}=p^{0}+p^{a} \omega_{a}-q_{a}^{\prime} \omega^{a}+q_{0}^{\prime} \omega_{\hat{\mathcal{X}}}
$$

where $\operatorname{ch}(E)$ and $\operatorname{Td}(\hat{\mathcal{X}})$ are the Chern character of $E$ and Todd class of $T \hat{\mathcal{X}}$. Using

$$
\begin{aligned}
& \mathrm{ch}=\mathrm{rk}+c_{1}+\left(\frac{1}{2} c_{1}^{2}-c_{2}\right)+\frac{1}{2}\left(c_{3}-c_{1} c_{2}+\frac{1}{3} c_{1}^{3}\right)+\ldots \\
& \mathrm{Td}=1+\frac{1}{2} c_{1}+\frac{1}{12}\left(c_{1}^{2}+c_{2}\right)+\frac{1}{24} c_{1} c_{2} \cdots=e^{c_{1} / 2} \hat{A},
\end{aligned}
$$

where $\hat{A}$ is the Dirac genus, the coefficients $p^{\Lambda}, q_{\Lambda}^{\prime}$ of $\gamma^{\prime}$ on the symplectic basis $\omega_{\Lambda}, \omega^{\Lambda}$ are found to be [84]

$$
\begin{aligned}
p^{a}=\int_{\gamma^{a}} c_{1}(E), \quad q_{a}^{\prime} & =-\left(\int_{\gamma_{a}} \operatorname{ch}_{2}(E)+\frac{p^{0}}{24} c_{2, a}\right), \\
q_{0}^{\prime} & =\int_{\hat{\mathcal{X}}}\left(\operatorname{ch}_{3}(E)+\frac{1}{24} c_{1}(E) c_{2}(\hat{\mathcal{X}})\right) .
\end{aligned}
$$

\footnotetext{
${ }^{27}$ We restore the important quadratic contribution $\frac{1}{2} A_{\Lambda \Sigma} X^{\Lambda} X^{\Sigma}$, which was omitted in [18]. Equivalently, one may extend the range of indices for the cubic form $\kappa_{a b c}$ and define $\kappa_{000}=-3 A_{00}, \kappa_{00 a}=-2 A_{0 a}, \kappa_{0 a b}=$ $-A_{a b}$.

${ }^{28}$ The prime anticipates the fact that the vector $\gamma^{\prime}$ lies in $H^{\text {even }}(\hat{\mathcal{X}}, \mathbb{Q})$. In eq. $(4.20)$ we introduce a "modified Mukai vector" which lies in $H^{\text {even }}(\hat{\mathcal{X}}, \mathbb{Z})$.
} 
Due to the fractional coefficients appearing in (4.8) and in $\operatorname{ch}_{n}(E)$, the generalized Mukai vector $\gamma^{\prime}=\left(p^{\Lambda}, q_{\Lambda}^{\prime}\right)$ is not valued in $H^{\text {even }}(\hat{\mathcal{X}}, \mathbb{Z})$ but rather in $H^{\text {even }}(\hat{\mathcal{X}}, \mathbb{Q})$. This appears to challenge the fact that the D2-brane charges in type IIA compactified on the mirror 3 -fold $\mathcal{X}$ are classified by $H_{3}(\mathcal{X}, \mathbb{Z})$ modulo torsion. To find the correct integer-valued charge vector $\gamma=\left(p^{\Lambda}, q_{\Lambda}\right)$, it suffices to match the central charge $\int_{\hat{\mathcal{X}}} e^{-z^{a} \omega_{a}} \gamma^{\prime}$ associated to the sheaf $F$ with the holomorphic supergravity central charge $e^{-\mathcal{K} / 2} Z_{\gamma}=q_{\Lambda} X^{\Lambda}-p^{\Lambda} F_{\Lambda}$, leading to

$$
q_{\Lambda}=q_{\Lambda}^{\prime}+A_{\Lambda \Sigma} p^{\Sigma}
$$

Thus, $q_{\Lambda}$ and $q_{\Lambda}^{\prime}$ differ by a (non-integer) symplectic transformation, which removes the real quadratic terms in (4.4), transforming the prepotential $F$ into

$$
F^{\prime}(X)=F(X)-\frac{1}{2} A_{\Lambda \Sigma} X^{\Lambda} X^{\Sigma} .
$$

The magnetic charges $p^{0}$ and $p^{a}$ are manifestly integer. To see that the electric charges $q_{\Lambda}$ are integer, it is convenient to rewrite them as

$$
\begin{aligned}
& q_{a}=\int_{\gamma_{a}} c_{2}(E)+p^{0}\left(A_{0 a}-\frac{c_{2, a}}{24}\right)+A_{a b} p^{b}-\frac{1}{2} \kappa_{a b c} p^{b} p^{c}, \\
& q_{0}=\left(\int_{\hat{\mathcal{X}}} \operatorname{ch}(E) \operatorname{Td}(\hat{\mathcal{X}})\right)+p^{a}\left(A_{0 a}-\frac{c_{2, a}}{24}\right)+A_{00} p^{0} .
\end{aligned}
$$

Observing that the first term in $q_{0}$ is the index of the Dirac operator coupled to the sheaf $F$, and therefore an integer, ${ }^{29}$ it follows that $q_{\Lambda} \in \mathbb{Z}$ provided the following additional constraints are imposed on the matrix $A_{\Lambda \Sigma}$ :

$$
\begin{aligned}
& \text { i) } A_{a b} p^{b}-\frac{1}{2} \kappa_{a b c} p^{b} p^{c} \in \mathbb{Z} \quad \text { for } \forall p^{a} \in \mathbb{Z}, \\
& \text { ii) } A_{0 a} \in \frac{c_{2, a}}{24}+\mathbb{Z} \\
& \text { iii) } A_{00} \in \mathbb{Z}
\end{aligned}
$$

The last condition shows that $A_{00}$ can be chosen to vanish. The second condition was conjectured long ago in [85] on the basis of a few examples, and is now seen to follow from K-theory considerations. Without loss of generality we shall choose $A_{0 a}=c_{2 a} / 24$. The first condition, which appears to be novel, requires that $2 A_{a b}$ is integer. It indeed holds true in the few examples where $A_{\Lambda \Sigma}$ has actually been computed [55-58]. For later reference, we note that the integrality of the Dirac operator on $\hat{\mathcal{X}}$ coupled to a rank one sheaf $\left(c_{2}(E)=c_{3}(E)=0\right)$ further requires ${ }^{30}$

$$
\text { iv) } N\left(p^{a}\right)+\frac{1}{12} c_{2, a} p^{a} \in \mathbb{Z} \quad \text { for } \forall p^{a} \in \mathbb{Z} .
$$

In view of $i$ ) and $i v$ ), it will be useful to define the integer-valued combinations

$$
L_{0}\left(p^{a}\right)=N\left(p^{a}\right)+\frac{1}{12} c_{2, a} p^{a}, \quad L_{a}\left(p^{a}\right)=\frac{1}{2} \kappa_{a b c} p^{b} p^{c}-A_{a b} p^{b} .
$$

\footnotetext{
${ }^{29}$ We are grateful to R. Minasian for discussions on related issues.

${ }^{30}$ This condition also guarantees that the dimension of the moduli space of the divisor Poincaré dual to $c_{1}(E)$ be even [86].
} 
Assuming that these integrality conditions are obeyed (as they must for mirror symmetry to hold), the charge vector $\gamma=\left(p^{\Lambda}, q_{\Lambda}\right)$ is now valued in $H^{\text {even }}(\mathcal{X}, \mathbb{Z})$, and can be meaningfully identified with the homology class of a special Lagrangian submanifold on the mirror type IIA side. On the other hand, the primed charges (4.8) satisfy

$$
q_{a}^{\prime} \in \mathbb{Z}-\frac{p^{0}}{24} c_{2, a}-\frac{1}{2} \kappa_{a b c} p^{b} p^{c}, \quad q_{0}^{\prime} \in \mathbb{Z}-\frac{1}{24} p^{a} c_{2, a} .
$$

For $p^{0}=0$, we recover the quantization conditions $q_{a}^{\prime} \in \mathbb{Z}-\frac{1}{2} \kappa_{a b c} p^{b} p^{c}$ and $q_{0}^{\prime} \in \mathbb{Z}-\frac{1}{24} p^{a} c_{2, a}$ familiar from studies of the D4-D2-D0 brane partition function [72, 73, 87, 88]. As we shall see in section 4.2, the fractional shifts in (4.15) have important implications for S-duality.

\subsubsection{Symplectic Jacobian and mirror symmetry}

We are now finally ready to relate the variables $\left(\zeta^{\Lambda}, \tilde{\zeta}_{\Lambda}\right)$ appearing in the metric (3.1) to the periods of the type IIB RR form $A^{\text {even }}$, and to specify the torus bundle $\mathcal{T} \rightarrow \mathcal{J}_{K}(\hat{\mathcal{X}}) \rightarrow$ $\mathcal{M}_{K}(\hat{\mathcal{X}})$. For this purpose, recall that the classical action of the D-instanton associated to the sheaf $E$ is given by $[46,89]$

$$
S_{\gamma}=8 \pi e^{(\phi+\mathcal{K}) / 2}\left|\int_{\hat{\mathcal{X}}} e^{-\mathcal{J}} \gamma^{\prime}\right|+2 \pi \mathrm{i} \int_{\hat{\mathcal{X}}} \gamma^{\prime} \wedge A^{\text {even }} e^{-B} .
$$

Identifying the periods of the RR potential $A^{\text {even }}$ as

$$
A^{\text {even }} e^{-B}=\zeta^{0}-\zeta^{a} \omega_{a}-\tilde{\zeta}_{a}^{\prime} \omega^{a}-\tilde{\zeta}_{0}^{\prime} \omega_{\hat{\mathcal{X}}}
$$

and defining the unprimed axions

$$
\tilde{\zeta}_{\Lambda}=\tilde{\zeta}_{\Lambda}^{\prime}+A_{\Lambda \Sigma} \zeta^{\Lambda}
$$

we find that the Euclidean action (4.16) associated to the D5-D3-D1-D(-1) bound state with integer charges $\left(p^{\Lambda}, q_{\Lambda}\right)$ becomes equal to the Euclidean action (3.7) of a D2-brane wrapping a sLag in the integer homology class $q_{\Lambda} \mathcal{A}^{\Lambda}-p^{\Lambda} \mathcal{B}_{\Lambda} \in H_{3}(\mathcal{X}, \mathbb{Z})$ on the mirror threefold $\mathcal{X}$. In particular, the coordinates $\left(\zeta^{\Lambda}, \tilde{\zeta}_{\Lambda}\right)$ defined by $(4.17),(4.18)$ have unit periodicity, and parametrize the torus $\mathcal{T}$.

Under a monodromy $M$ in the moduli space of Kähler structures $\mathcal{M}_{K}(\hat{\mathcal{X}})$, the torus transforms by a symplectic rotation $\rho(M) \in \operatorname{Sp}\left(2 b_{2}(\hat{\mathcal{X}})+2, \mathbb{Z}\right)$ computable from the prepotential $F(X)$ (see section 4.1.4). Thus, the symplectic Jacobian $\mathcal{J}_{K}(\hat{\mathcal{X}})$ is the total space of the torus bundle

$$
\begin{aligned}
H^{\text {even }}(\hat{\mathcal{X}}, \mathbb{R}) / \Gamma \rightarrow & \mathcal{J}_{K}(\hat{\mathcal{X}}) \\
& \downarrow \\
& \mathcal{M}_{K}(\hat{\mathcal{X}}),
\end{aligned}
$$

where the lattice $\Gamma \subset H^{\text {even }}(\hat{\mathcal{X}}, \mathbb{Z})$, dual to the lattice of D5-D3-D1-D(-1) brane charges, is the image of the K-theory lattice $K(\hat{\mathcal{X}})$ under the "modified Mukai map"

$$
E \mapsto \gamma=e^{A} \operatorname{ch}(E) \sqrt{\operatorname{Td}(\hat{\mathcal{X}})} \in H^{\text {even }}(\hat{\mathcal{X}}, \mathbb{Z}),
$$

where $e^{A}$ represents the fractional symplectic transformation (4.9). 
Having specified the torus bundle $\mathcal{J}_{K}(\hat{\mathcal{X}})$, the topology of the type IIB perturbative HM moduli space $\mathcal{M}$ follows by the same reasoning as in section $3: \mathcal{M}$ is foliated by hypersurfaces $\mathcal{C}(r)$ of constant coupling, each of which is a circle bundle $\mathcal{C}(r)$ over $\mathcal{J}_{K}(\hat{\mathcal{X}})$, with curvature given by

$$
\mathrm{d}\left(\frac{D \sigma}{2}\right)=\omega_{\mathcal{T}}-\frac{\chi(\hat{\mathcal{X}})}{24} \omega_{\mathcal{S K}}
$$

where $\omega_{\mathcal{S K}}$ is the Kähler class of $\mathcal{M}_{K}(\hat{\mathcal{X}})$, and the characteristics $\Theta$ which remain to be specified. This is in full agreement with the quantum mirror symmetry conjecture. While classical mirror symmetry identifies $\mathcal{M}_{K}(\hat{\mathcal{X}})=\mathcal{M}_{c}(\mathcal{X})$, semi-classical mirror symmetry (in its weakest form) demands $\mathcal{J}_{K}(\hat{\mathcal{X}})=\mathcal{J}_{c}(\mathcal{X})$, quantum mirror symmetry requires the identity of the full HM moduli spaces $\mathcal{Q}_{c}(\mathcal{X})=\mathcal{Q}_{K}(\hat{\mathcal{X}})$ as quaternion-Kähler manifolds.

\subsubsection{Monodromy around the large volume point}

We mentioned previously that the torus $\mathcal{T}$ is non-trivially fibered over $\mathcal{M}_{K}(\hat{\mathcal{X}})$. Here we consider the monodromy $M$ :

$$
b^{a} \mapsto b^{a}+\epsilon^{a}, \quad \epsilon^{a} \in \mathbb{Z}
$$

around the large volume point in $\mathcal{M}_{K}(\hat{\mathcal{X}})$. This monodromy must be accompanied by the symplectic rotation

$$
\begin{aligned}
& \zeta^{a} \mapsto \zeta^{a}+\epsilon^{a} \zeta^{0}, \quad \tilde{\zeta}_{a}^{\prime} \mapsto \tilde{\zeta}_{a}^{\prime}-\kappa_{a b c} \zeta^{b} \epsilon^{c}-\frac{1}{2} \zeta^{0} \kappa_{a b c} \epsilon^{b} \epsilon^{c}, \\
& \tilde{\zeta}_{0}^{\prime} \mapsto \tilde{\zeta}_{0}^{\prime}-\epsilon^{a} \tilde{\zeta}_{a}^{\prime}+\frac{1}{2} \kappa_{a b c} \zeta^{a} \epsilon^{b} \epsilon^{c}+\frac{1}{6} \zeta^{0} \kappa_{a b c} \epsilon^{a} \epsilon^{b} \epsilon^{c}
\end{aligned}
$$

on the torus $\mathcal{T}$, as follows from the definition (4.17). Upon transforming the charges according to

$$
\begin{aligned}
p^{0} \mapsto p^{0}, \quad p^{a} & \mapsto p^{a}+\epsilon^{a} p^{0}, \quad q_{a}^{\prime} \mapsto q_{a}^{\prime}-\kappa_{a b c} p^{b} \epsilon^{c}-\frac{1}{2} p^{0} \kappa_{a b c} \epsilon^{b} \epsilon^{c}, \\
q_{0}^{\prime} & \mapsto q_{0}^{\prime}-\epsilon^{a} q_{a}^{\prime}+\frac{1}{2} \kappa_{a b c} p^{a} \epsilon^{b} \epsilon^{c}+\frac{1}{6} p^{0} \kappa_{a b c} \epsilon^{a} \epsilon^{b} \epsilon^{c},
\end{aligned}
$$

the instanton action (4.16) remains invariant. The transformation (4.24) amounts to tensoring the sheaf $E$ with a line bundle $E^{\prime}$ with first Chern class $c_{1}\left(E^{\prime}\right)=\epsilon^{a} \omega_{a}$. We refer to the transformation (4.24) as a "spectral flow" transformation with parameter $\epsilon^{a}$, and the transformed (unprimed) charge vector as $\gamma[\epsilon]$. The spectral flow (4.24) can be rewritten as an integral symplectic transformation of the integral charge vector $\gamma$,

$$
\gamma[\epsilon]=\rho(M) \cdot \gamma, \quad \rho(M)=\left(\begin{array}{cccc}
1 & 0 & 0 & 0 \\
\epsilon^{a} & \delta^{a}{ }_{b} & 0 & 0 \\
-L_{a}(\epsilon) & -\kappa_{a b c} \epsilon^{c} & \delta_{a}^{b} & 0 \\
L_{0}(\epsilon) & L_{b}(\epsilon)+2 A_{b c} \epsilon^{c} & -\epsilon^{b} & 1
\end{array}\right),
$$

which makes it clear that the spectral flow preserves the quantization conditions (4.15). 
For $p^{0} \neq 0$, the combinations

$$
\hat{q}_{a}=q_{a}^{\prime}+\frac{1}{2} \kappa_{a b c} \frac{p^{b} p^{c}}{p^{0}}, \quad \hat{q}_{0}=q_{0}^{\prime}+\frac{p^{a} q_{a}^{\prime}}{p^{0}}+\frac{1}{3} \kappa_{a b c} \frac{p^{a} p^{b} p^{c}}{\left(p^{0}\right)^{2}}
$$

are invariant under the spectral flow (4.24). For a fixed $p^{0}$, we shall refer to $\hat{\gamma}=\left(p^{a}, \hat{q}_{a}, \hat{q}_{0}\right)$ as the reduced charge vector. The invariant charges $\hat{q}_{\Lambda}$ may be expressed in terms of the Chern classes of $E$ as

$$
\begin{aligned}
& \hat{q}_{a}=\int_{\gamma_{a}}\left[c_{2}(E)+\frac{1-p^{0}}{2 p^{0}} c_{1}^{2}(E)\right]-\frac{p^{0}}{24} c_{2, a}, \\
& \hat{q}_{0}=\int_{\hat{\mathcal{X}}}\left[\operatorname{ch}_{3}(E)-\frac{1}{p^{0}} c_{1}(E) \operatorname{ch}_{2}(E)+\frac{1}{3\left(p^{0}\right)^{2}} c_{1}^{3}(E)\right] .
\end{aligned}
$$

These combinations coincide (up to the last term in $\hat{q}_{a}$ ) with the homogeneous invariants of [84]. In particular, $\hat{q}_{a}+\frac{p^{0}}{24} c_{2, a}$ is recognized as the "Bogomolov discriminant". It is also useful to observe that

$$
Q_{a} \equiv p^{0}\left(\hat{q}_{a}+\frac{p^{0}}{24} c_{2, a}\right), \quad J \equiv \frac{1}{2}\left(p^{0}\right)^{2} \hat{q}_{0}
$$

are precisely (after matching conventions) the electric charge and angular momentum of the 5D lift of the 4D D6-D4-D2-D0 black hole with charges $\left(p^{0}, p^{a}, q_{a}^{\prime}, q_{0}^{\prime}\right)$ [90-92]. Moreover, for $p^{0}=1, Q_{a}$ and $2 J$ are integer. These observations will play a role in section 5.4.

While the combined action of the monodromy $M$ on the moduli via (4.22) and (4.23) and on the charges via (4.24) preserves the D-instanton action and stability condition, and therefore the generalized Donaldson-Thomas invariant $\Omega\left(\gamma, z^{a}\right)$, the latter is in general not invariant under $\gamma \mapsto \gamma[\epsilon]$ at fixed values of the moduli, due to wall-crossing phenomena. In the heuristic discussion of section 5, we shall however ignore this issue and assume that $\Omega(\gamma)$ is in fact invariant under spectral flow, at fixed values of the moduli.

\subsection{S-duality}

The type IIB description is more advantageous for dealing with non-perturbative corrections as it provides an infinite discrete symmetry mixing worldsheet instantons and D-instantons, the $10 \mathrm{D} \mathrm{SL}(2, \mathbb{Z})$ S-duality symmetry. ${ }^{31}$ In the zero coupling, infinite volume limit, where only the first, cubic term in the prepotential (4.4) is retained and the one-loop correction can be ignored, the moduli space $\mathcal{Q}_{K}(\hat{\mathcal{X}})$ admits an isometric action of $\operatorname{SL}(2, \mathbb{R})[14,18,93]$, corresponding to the S-duality of 10-dimensional type IIB string theory. This action is most easily described using new coordinates $\left(\tau_{1}, c^{a}, c_{a}, c_{0}, \psi\right)$ related

\footnotetext{
${ }^{31}$ The same $\operatorname{SL}(2, \mathbb{Z})$ action is also manifest geometrically in the context of M-theory on $\hat{\mathcal{X}} \times T^{2}$, whose VM moduli space is given by the same space $\mathcal{Q}_{K}(\hat{\mathcal{X}})$. As already pointed out in the introduction, it is debatable whether the full $\mathrm{SL}(2, \mathbb{Z})$ symmetry should stay unbroken in vacua with $\mathcal{N}=2$ supersymmetry, or whether it should be broken to a finite index subgroup. At this point we assume that it does, though our discussion can be adapted to accommodate the weaker option.
} 
to the coordinates $\left(\zeta^{\Lambda}, \tilde{\zeta}_{\Lambda}, \sigma\right)$ by

$$
\begin{array}{rlrl}
\zeta^{0} & =\tau_{1}, & \zeta^{a} & =-\left(c^{a}-\tau_{1} b^{a}\right), \\
\tilde{\zeta}_{a}^{\prime} & =c_{a}+\frac{1}{2} \kappa_{a b c} b^{b}\left(c^{c}-\tau_{1} b^{c}\right), & \tilde{\zeta}_{0}^{\prime} & =c_{0}-\frac{1}{6} \kappa_{a b c} b^{a} b^{b}\left(c^{c}-\tau_{1} b^{c}\right), \\
\sigma & =-2\left(\psi+\frac{1}{2} \tau_{1} c_{0}\right)+c_{a}\left(c^{a}-\tau_{1} b^{a}\right)-\frac{1}{6} \kappa_{a b c} b^{a} c^{b}\left(c^{c}-\tau_{1} b^{c}\right)
\end{array}
$$

and the ten-dimensional inverse coupling $\tau_{2}=1 / g_{s}$ related to the $4 \mathrm{D}$ dilaton by [18]

$$
e^{\phi}=\frac{\tau_{2}^{2}}{16} e^{-\mathcal{K}(z, \bar{z})}-\frac{\chi(\hat{\mathcal{X}})}{192 \pi} .
$$

Then, an element $\delta=\left(\begin{array}{ll}a & b \\ c & d\end{array}\right) \in \mathrm{SL}(2, \mathbb{R})$ acts by fractional linear transformations of the axio-dilaton field $\tau \equiv \tau_{1}+\mathrm{i} \tau_{2}$, rescaling of the Kähler moduli and by linear action on the RR and NS axions:

$$
\begin{aligned}
& \tau \mapsto \frac{a \tau+b}{c \tau+d}, \quad t^{a} \mapsto t^{a}|c \tau+d|, \quad c_{a} \mapsto c_{a}+\varepsilon_{a}(\delta), \\
& \left(\begin{array}{l}
c^{a} \\
b^{a}
\end{array}\right) \mapsto\left(\begin{array}{ll}
a & b \\
c & d
\end{array}\right)\left(\begin{array}{l}
c^{a} \\
b^{a}
\end{array}\right), \quad\left(\begin{array}{l}
c_{0} \\
\psi
\end{array}\right) \mapsto\left(\begin{array}{cc}
d & -c \\
-b & a
\end{array}\right)\left(\begin{array}{l}
c_{0} \\
\psi
\end{array}\right) .
\end{aligned}
$$

Here we deviate from $[14,18,93]$, and allow for a shift in the D3-brane axion $c_{a}$ by an additive character $\varepsilon_{a}(\delta)$ of $\operatorname{SL}(2, \mathbb{R})$, i.e. such that $\varepsilon_{a}\left(\delta \cdot \delta^{\prime}\right)=\varepsilon_{a}(\delta)+\varepsilon_{a}\left(\delta^{\prime}\right)$.

The continuous isometric action (4.31) is broken by the worldsheet instanton effects in (4.4) and the one-loop correction $c$, but a discrete $\operatorname{SL}(2, \mathbb{Z})$ subgroup can be restored by including $\mathrm{D}(-1)$ and D1-brane instantons [21]. This in particular provides a common origin $^{32}$ for the degenerate instanton contribution, proportional to $\zeta(3) \chi(\hat{\mathcal{X}})$ in $(4.4)$ and the one-loop correction, proportional to $\zeta(2) \chi(\hat{\mathcal{X}})$. The analysis in [21] did not probe the possibility of an additional character $\varepsilon_{a}(\delta)$.

In the presence of D3-brane instantons, the character $\varepsilon_{a}(\delta)$ is needed for the following reason. Due to the fractional shift (4.15) of the $\mathrm{D}(-1)$-brane charge $q_{0}^{\prime}$, a D-instanton contribution proportional to $\mathbf{E}\left(p^{\Lambda} \tilde{\zeta}_{\Lambda}-q_{\Lambda} \zeta^{\Lambda}\right)=\mathbf{E}\left(p^{\Lambda} \tilde{\zeta}_{\Lambda}^{\prime}-q_{\Lambda}^{\prime} \zeta^{\Lambda}\right)$ transforms under an S-duality action $\delta_{b}=\left(\begin{array}{ll}1 & b \\ 0 & 1\end{array}\right)$ by a phase $\mathbf{E}\left(\frac{p^{a} c_{2, a}}{24} b+p^{a} \varepsilon_{a}\left(\delta_{b}\right)\right)$. Moreover, this S-duality action differs from the Heisenberg shift (3.15) with parameter $\eta^{0}=b$ by a fractional shift $\tilde{\zeta}_{a} \mapsto \tilde{\zeta}_{a}+\varepsilon_{a}\left(\delta_{b}\right)+\frac{c_{2, a}}{24} b$. Both these problems can be avoided by choosing the character $\varepsilon_{a}(\delta)$ such that $\varepsilon_{a}\left(\delta_{b}\right)=-\frac{c_{2, a}}{24} b$. This is indeed the case if $\varepsilon_{a}$ is taken to be proportional to the multiplier system of the Dedekind eta function,

$$
\varepsilon_{a}(\delta)=-c_{2, a} \varepsilon(\delta),
$$

where $\varepsilon(\delta)$ is a fractional number defined, up to the addition of integers, by the ratio ${ }^{33}$

$$
\eta\left(\frac{a \tau+b}{c \tau+d}\right) / \eta(\tau)=\mathbf{E}(\varepsilon(\delta))(c \tau+d)^{1 / 2} .
$$

\footnotetext{
${ }^{32}$ In fact, this was used in [21] to confirm the normalization of $c$ in (3.3).

${ }^{33}$ Strictly speaking, the two factors on the r.h.s. of (4.33) both have sign ambiguities, and only their product is well-defined. But since the condition (4.13) ensures that $c_{2, a}$ is even, one only requires the value of $\varepsilon(\delta)$ modulo $1 / 2$.
} 
In particular, $24 \varepsilon(\delta)$ is integer, and may be obtained explicitly as

$$
\varepsilon(\delta)=\left\{\begin{array}{cc}
\frac{b}{24} \operatorname{sgn}(d) & (c=0), \\
\frac{a+d}{24 c}-\frac{1}{2} s(d, c)-\frac{1}{8} & (c \neq 0),
\end{array}\right.
$$

where

$$
s(d, c)=\frac{1}{4|c|} \sum_{n=1}^{|c|-1} \cot \frac{\pi n}{c} \cot \frac{\pi n d}{c}
$$

is the Dedekind sum. The identification (4.32) is also supported by the modular properties of the D4-D2-D0 partition function [73, 87, 88, 94, 95], which transforms with the multiplier system $\mathbf{E}\left(-c_{2, a} p^{a} \varepsilon(\delta)\right) \quad[73,94] .{ }^{34}$

\subsection{Semi-classical $(p, k) 5$-brane instantons}

Having rectified the action of S-duality on the large volume, weak coupling HM moduli space, we can now use it to obtain NS5-brane instantons from D-instantons. Indeed, from the action on $\left(c_{0}, \psi\right)$ we see that S-duality maps D5-branes, with minimal coupling to the D5 axion $c_{0}$, to NS5-branes coupled to the NS-axion $\psi$, or more generally to $(p, k) 5$ branes coupled to $p c_{0}+k \psi$. In particular, since $c_{0}=\tilde{\zeta}_{0}+\ldots$ has unit periodicity and $\psi=-\frac{1}{2} \sigma+\ldots$, we conclude that the NS-axion $\sigma$ must have periodicity 2 , as anticipated in section 3.2.2.

Let us now consider a configuration of D5-D3-D1-D(-1) instantons with charges $\left(p^{0} \neq\right.$ $\left.0, p^{a}, q_{a}, q_{0}\right)$, discussed in detail in the previous subsection. The classical action associated to this charge configuration is

$$
S_{\gamma}=4 \pi\left|W_{\gamma}\right|+2 \pi \mathrm{i}\left(q_{\Lambda}^{\prime} \zeta^{\Lambda}-p^{\Lambda} \tilde{\zeta}_{\Lambda}^{\prime}\right)
$$

where

$$
W_{\gamma}=\frac{\tau_{2}}{2}\left(q_{\Lambda}^{\prime} z^{\Lambda}-p^{\Lambda} F_{\Lambda}^{\prime}(z)\right)
$$

is proportional to the central charge $Z_{\gamma}=e^{\mathcal{K} / 2}\left(q_{\Lambda}^{\prime} X^{\Lambda}-p^{\Lambda} F_{\Lambda}^{\prime}\right)$ of the D-instanton. In the large volume limit where the cubic term in (4.4) dominates,

$$
W_{\gamma}=\frac{\tau_{2}}{2}\left(\frac{N\left(p^{a}-p^{0} z^{a}\right)}{\left(p^{0}\right)^{2}}-\frac{\hat{q}_{a}\left(p^{a}-p^{0} z^{a}\right)}{p^{0}}+\hat{q}_{0}\right) .
$$

For any two integers $(p, k) \neq(0,0)$, with greatest common divisor (gcd) $p^{0}$, we now apply an S-duality transformation

$$
\delta=\left(\begin{array}{cc}
a & b \\
-k / p^{0} & p / p^{0}
\end{array}\right) \in \mathrm{SL}(2, \mathbb{Z}),
$$

where the integers $(a, b)$, ambiguous up to the addition of $\left(k / p^{0},-p / p^{0}\right)$, are chosen such that $a p+b k=p^{0}$. This will map the D5-brane configuration into a $(p, k) 5$-brane.

\footnotetext{
${ }^{34}$ We are grateful to J. Manschot for discussions on this issue.
} 
Using (4.31), one finds that the image of $W_{\gamma}$ under the map (4.39) is given by

$$
W_{k, p, \hat{\gamma}} \equiv \delta \cdot W_{\gamma}=\frac{\tau_{2}}{2|p-k \tau|^{2}}\left[N\left(\tilde{p}^{a}-i|p-k \tau| t^{a}\right)-p^{0} \hat{q}_{a}\left(\tilde{p}^{a}-\mathrm{i}|p-k \tau| t^{a}\right)+\left(p^{0}\right)^{2} \hat{q}_{0}\right],
$$

where we recall that $\hat{\gamma}$ is the reduced charge vector $\left(p^{a}, \hat{q}_{a}, \hat{q}_{0}\right)$ and $\tilde{p}^{a} \equiv p^{a}+k c^{a}-p b^{a}$. As a result, the action (4.36) is mapped to $S_{k, p, \hat{\gamma}}$ which reads as follows

$$
\begin{aligned}
S_{k, p, \hat{\gamma}}= & 4 \pi\left|W_{k, p, \hat{\gamma}}\right|+2 \pi \mathrm{i}\left[-\left(p c_{0}+k \psi+p^{a} c_{a}\right)+\frac{p-k \tau_{1}}{k|p-k \tau|^{2}}\left(N\left(\tilde{p}^{a}\right)-p^{0} \hat{q}_{a} \tilde{p}^{a}+\left(p^{0}\right)^{2} \hat{q}_{0}\right)\right. \\
& \left.+\frac{b^{a}}{2 k}\left(\frac{1}{3} \kappa_{a b c}\left(p b^{b}-k c^{b}\right)\left(3 \tilde{p}^{c}+p b^{c}-k c^{c}\right)+\kappa_{a b c} \tilde{p}^{b} \tilde{p}^{c}-2 p^{0} \hat{q}_{a}\right)-\frac{a}{k} q_{0}^{\prime} p^{0}+c_{2, a} p^{a} \epsilon(\delta)\right] .
\end{aligned}
$$

It should be stressed that the unusual additional terms in the imaginary part of $S_{k, p, \hat{\gamma}}$ follow from the ordinary axionic couplings in (4.36) by S-duality, and evade the no-go result of [96]. This is eventually tied to the impossibility of defining instanton vacua with well defined mutually non-local D-instanton charges in the presence of NS5-brane instantons. Notice that the last two moduli-independent terms in (4.41) are the only ones depending explicitly on the upper entries in the S-duality matrix (4.39). What is important however is that their combination is independent of the choice of these entries so that the action is well defined. Although some terms are singular at $k=0$, but the total sum in (4.41) is regular at $k=0$, where it reduces to (4.36). Moreover, for $\hat{q}_{a}=\hat{q}_{0}=0$, the action reduces to the answer found in eq. (3.72) of [54], based on the assumption that the S-duality group is extended to $\mathrm{SL}(3, \mathbb{Z})$.

From (4.41), it is now apparent that $\psi$ is periodic with period 1, and therefore $\sigma$ is periodic with period 2. Moreover, in the weak coupling limit $\tau_{2} \rightarrow \infty$ and using the form of the period matrix $\mathcal{N}$ for a cubic prepotential,

$$
\mathcal{N}_{\Lambda \Sigma}=\left(\begin{array}{cc}
-\frac{1}{3}(b b b) & \frac{1}{2}(b b)_{a} \\
\frac{1}{2}(b b)_{a} & -\kappa_{a b c} b^{c}
\end{array}\right)+\mathrm{i}\left(\begin{array}{cc}
-\frac{1}{6}(t t t)+(b b t)-\frac{1}{4 V}(b t t)^{2} & -(b t)_{a}+\frac{1}{4 V}(b t t)(t t)_{a} \\
-(b t)_{a}+\frac{1}{4 V}(b t t)(t t)_{a} & \kappa_{a b c} t^{c}-\frac{1}{4 V}(t t)_{a}(t t)_{b}
\end{array}\right),
$$

where we introduced the notation $(x y z)=\kappa_{a b c} x^{a} y^{b} z^{c},(x y)_{a}=\kappa_{a b c} x^{b} y^{c}$, we find that the action for $k$ NS5-branes reduces to

$$
S_{k, p, \hat{\gamma}}=2 \pi|k| V \tau_{2}^{2}+\pi \mathrm{i} k\left(\sigma+\zeta^{\Lambda} \tilde{\zeta}_{\Lambda}^{\prime}-2 n^{\Lambda} \tilde{\zeta}_{\Lambda}^{\prime}-\overline{\mathcal{N}}_{\Lambda \Sigma}\left(\zeta^{\Lambda}-n^{\Lambda}\right)\left(\zeta^{\Sigma}-n^{\Sigma}\right)\right)-2 \pi \mathrm{i} m_{\Lambda} z^{\Lambda}
$$

Here we defined

$$
n^{0}=p / k, \quad n^{a}=p^{a} / k, \quad m_{a}=p^{0} \hat{q}_{a} / k, \quad m_{0}=a p^{0} q_{0}^{\prime} / k-c_{2, a} p^{a} \epsilon(\delta) .
$$

For $m_{\Lambda}=0$ this reproduces (3.9), and thus confirms the validity of (3.8).

\section{NS5-instantons in twistor space}

In the previous sections we discussed instanton corrections to the HM moduli space $\mathcal{M}$ of type II string theory on a Calabi-Yau threefold at a qualitative level, ignoring the 
tensorial nature of the metric on $\mathcal{M}$ and jettisoning the constraints of supersymmetry. The latter however require that all quantum corrections preserve the quaternion-Kähler property of the metric on $\mathcal{M}$ [4]. A convenient way to ensure this is to formulate the quantum corrections as deformations of the complex contact structure on the twistor space $\mathcal{Z}_{\mathcal{M}}$, a complex contact manifold locally of the form $\mathbb{C} P^{1} \times \mathcal{M}$.

In this section, we take steps towards identifying the deformation of the complex contact structure on $\mathcal{Z}_{\mathcal{M}}$ induced by NS5-brane instantons, by applying S-duality to the known twistorial description of D-instantons. We work in the one-instanton approximation, i.e. treat instanton corrections as an infinitesimal deformations of the complex contact structure on $\mathcal{Z}_{\text {pert }}$, the twistor space of the perturbative HM moduli space discussed in section 3. Infinitesimal deformations are encoded in a holomorphic section of $H^{1}\left(\mathcal{Z}_{\text {pert }}, \mathcal{O}(2)\right)$. In practice it is most convenient to represent this section as a set of local holomorphic functions defined on the overlap of two patches in a suitable open covering of $\mathbb{C} P^{1}$. In general, this set need only be invariant under discrete symmetries up to local contact transformations. Here we assume that this set is in fact simultaneously invariant under S-duality, Heisenberg invariance and monodromy transformations, without the need for any compensating contact transformation. This is the simplest approach to constructing a metric with the required discrete symmetries. While we find strong indications that this assumption is reasonable, we do encounter difficulties in realizing all symmetries simultaneously, due to certain phases spoiling exact invariance. As a result, sections 5.3 onward should be considered as exploratory, though we do believe that the structure we uncover should be realized in a fully consistent treatment.

The outline of this section is as follows. • In section 5.1, we briefly recall the twistorial description of general quaternion-Kähler manifolds, following $[12,18]$. • In section 5.2, we summarize the twistorial description of D-instanton corrections established in [18], taking into account the fractional shift (4.18) and quadratic refinement which had been overlooked in previous treatments. - In section 5.3, we obtain the twistorial description of $(p, k) 5$ brane instantons in type IIB string theory compactified on $\hat{\mathcal{X}}$, by applying S-duality on a general D5-D3-D1-D(-1) configuration. We show that the corresponding deformation of the contact structure is invariant under the Heisenberg shifts (3.15) and monodromy around infinity (4.23) (or rather their holomorphic lifts (5.12), (5.13) to the twistor space $\mathcal{Z}_{\text {pert }}$ ), up to subtle phases which we do not understand. - Under this caveat, we show in section 5.4 that the total contribution of fixed NS5-brane charge $k$ can be expressed as a non-Gaussian theta series with wave function $H_{k, \mu}$. For a single NS5-brane, $k=1, H_{1,0}$ is recognized (up to certain prefactors) as the A-model topological string wave function on $\hat{\mathcal{X}}$, in the real polarization. • Finally, in section 5.5 we obtain the non-Gaussian chiral partition function $\mathcal{Z}_{\text {NS5 }}^{(k)}$ for $k$ fivebranes as the Penrose transform of the holomorphic function encoding NS5brane corrections to the contact structure. In the saddle point approximation it reproduces the non-linear $(p, k)$-fivebrane action from section 4.3 , whereas the weak coupling limit of $\mathcal{Z}_{\text {NS5 }}^{(k)}$ reduces to a Gaussian partition function $\mathcal{Z}_{\text {NS5-G }}^{(k)}$ similar to (but distinct from) the Gaussian flux partition function $\mathcal{Z}_{\mathrm{G}}^{(k)}$ discussed in section 2 . 


\subsection{Twistor techniques}

Recall that a manifold $\mathcal{M}$ of real dimension $4 n>4$ is quaternion-Kähler if its holonomy group lies in $\operatorname{USp}(n) \times \mathrm{USp}(1) \subset \mathrm{SO}(4 n)$, where $\mathrm{USp}(1)=\mathrm{SU}(2)$ (in dimension 4, this condition must be replaced by the vanishing of the self-dual part of the Weyl curvature). While $\mathcal{M}$ admits locally a triplet of almost-complex structures $\vec{I}$ satisfying the quaternion algebra, these are not integrable. The space $\mathcal{M}$ is nevertheless amenable to complex analysis by considering its twistor space $\mathcal{Z}_{\mathcal{M}}$, a $\mathbb{C} P^{1}$ bundle over $\mathcal{M}$ which carries a canonical, integrable complex structure, Kähler-Einstein metric as well as a complex contact structure. To explain how this comes about, let $t$ be a complex stereographic coordinate on $\mathbb{C} P^{1}$, and $\vec{p}$ be the $\mathrm{SU}(2)$ part of the Levi-Civita connection on $\mathcal{M}$. Under $\mathrm{SU}(2)$ frame rotations, $\vec{p}$ transforms as a $\mathrm{SU}(2)$ gauge potential, while $t$ is acted upon by fractional linear transformations. The complex contact structure on $\mathcal{Z}_{\mathcal{M}}$ is defined by the kernel of the one-form

$$
D t \equiv \mathrm{d} t+p_{+}-\mathrm{i} p_{3} t+p_{-} t^{2}, \quad p_{ \pm}=-\frac{1}{2}\left(p_{1} \mp \mathrm{i} p_{2}\right) .
$$

Locally, on a patch $\mathcal{U}_{i} \subset \mathcal{Z}_{\mathcal{M}}$ there exists a function $\Phi_{[i]}$ on $\mathcal{Z}_{\mathcal{M}}$, holomorphic along the $\mathbb{C} P^{1}$-fiber (i.e. independent of $\bar{t}$ ) and defined up to an additive holomorphic function on $\mathcal{U}_{i}$, such that the product

$$
\mathcal{X}^{[i]}=2 e^{\Phi_{[i]}} \frac{D t}{\mathrm{i} t}
$$

is a locally holomorphic one-form (i.e. $\bar{\partial}$-closed). We refer to $\Phi_{[i]}$ as the contact potential. $\Phi_{[i]}$ determines a Kähler potential [12]

$$
K_{\mathcal{Z}_{\mathcal{M}}}^{[i]}=\log \frac{1+t \bar{t}}{|t|}+\operatorname{Re} \Phi_{[i]}\left(x^{\mu}, t\right)
$$

for the canonical Kähler-Einstein metric on $\mathcal{Z}_{\mathcal{M}}$

$$
\mathrm{d} s_{\mathcal{Z}_{\mathcal{M}}}^{2}=\frac{|D t|^{2}}{(1+t \bar{t})^{2}}+\frac{\nu}{4} \mathrm{~d} s_{\mathcal{M}}^{2}
$$

(here $\nu$ is a normalization constant which determines the scalar curvature of $\mathcal{M}$ ). Moreover, $\mathcal{Z}_{\mathcal{M}}$ is endowed with a real structure acting as the antipodal map on $\mathbb{C} P^{1}$, and preserving $\mathcal{X}$.

According to theorems by Salamon and Lebrun [97, 98], the complex contact structure and real structure on $\mathcal{Z}_{\mathcal{M}}$ uniquely specify the quaternion-Kähler metric on $\mathcal{M}$. Locally, one may always choose complex Darboux coordinates $\xi_{[i]}^{\Lambda}, \tilde{\xi}_{\Lambda}^{[i]}, \alpha^{[i]}(\Lambda=0, \ldots, n-1)$ on $\mathcal{U}_{i} \subset \mathcal{Z}_{\mathcal{M}}$ such that the contact one-form $\mathcal{X}$ takes the Darboux form

$$
\mathcal{X}^{[i]}=\mathrm{d} \alpha^{[i]}+\xi_{[i]}^{\Lambda} \mathrm{d} \tilde{\xi}_{\Lambda}^{[i]} .
$$

We shall find it convenient to define a variant of this coordinate system, with $\tilde{\alpha}^{[i]}=$ $-2 \alpha^{[i]}-\xi_{[i]}^{\Lambda} \tilde{\xi}_{\Lambda}^{[i]}$ such that the contact one-form takes the symmetric Darboux form

$$
\mathcal{X}^{[i]}=-\frac{1}{2}\left(\mathrm{~d} \tilde{\alpha}^{[i]}-\xi_{[i]}^{\Lambda} \mathrm{d} \tilde{\xi}_{\Lambda}^{[i]}+\tilde{\xi}_{\Lambda}^{[i]} \mathrm{d} \xi_{[i]}^{\Lambda}\right)
$$


On the overlap of two patches $\mathcal{U}_{i} \cap \mathcal{U}_{j}$, the Darboux coordinates are related by complex contact transformations preserving $\mathcal{X}^{[i]}$ up to holomorphic rescaling. Thus, the complex contact structure on $\mathcal{Z}_{\mathcal{M}}$ may be specified globally by giving generating functions $S^{[i j]}\left(\xi_{[i]}^{\Lambda}, \tilde{\xi}_{\Lambda}^{[j]}, \alpha^{[j]}\right)$ of these complex contact transformations. These must satisfy the obvious reality conditions and compatibility constraints, and are themselves defined up to independent complex contact transformations on each patch.

The quaternion-Kähler metric on $\mathcal{M}$ can be obtained by solving the gluing conditions for the Darboux coordinates on $\mathcal{U}_{i} \cap \mathcal{U}_{j}$ following from the generating functions $S^{[i j]}$,

$$
\begin{aligned}
\xi_{[j]}^{\Lambda} & =f_{i j}^{-2} \partial_{\tilde{\xi}_{\Lambda}^{[j]}} S^{[i j]}, & \tilde{\xi}_{\Lambda}^{[i]} & =\partial_{\xi_{[i]}^{\Lambda}} S^{[i j]}, \\
\alpha^{[i]} & =S^{[i j]}-\xi_{[i]}^{\Lambda} \partial_{\xi_{[i]}^{\Lambda}} S^{[i j]}, & e^{\Phi[i]} & =f_{i j}^{2} e^{\Phi_{[j]}},
\end{aligned}
$$

where $f_{i j}^{2}=\partial_{\alpha[j]} S^{[i j]}=\mathcal{X}^{[i]} / \mathcal{X}^{[j]}$ is the homogeneity factor relating the contact one-forms. The parameter space $\left\{x^{\mu}\right\}$ of solutions is $\mathcal{M}$ itself, while the solutions $\xi_{[i]}^{\Lambda}\left(t ; x^{\mu}\right), \tilde{\xi}_{\Lambda}^{[i]}\left(t ; x^{\mu}\right)$ and $\alpha^{[i]}\left(t ; x^{\mu}\right)$ can be plugged in (5.5) to read off the $\mathrm{SU}(2)$ connection in (5.1). The metric itself follows after some more steps explained in [12].

Furthermore, infinitesimal perturbations around a given quaternion-Kähler manifold preserving the quaternion-Kähler structure are in one-to-one correspondence with $H^{1}\left(\mathcal{Z}_{\mathcal{M}}, \mathcal{O}(2)\right)$ [99]. They can be parametrized explicitly as a set of local holomorphic functions $H_{(1)}^{[i j]}$ on the overlap of two patches $\mathcal{U}_{i} \cap \mathcal{U}_{j}$, corresponding to the variation of the quantity $H^{[i j]}$ entering in the generating function $S^{[i j]}$ via [12]

$$
S^{[i j]}\left(\xi_{[i]}^{\Lambda}, \tilde{\xi}_{\Lambda}^{[j]}, \alpha^{[j]}\right)=\alpha^{[j]}+\xi_{[i]}^{\Lambda} \tilde{\xi}_{\Lambda}^{[j]}-H^{[i j]}\left(\xi_{[i]}^{\Lambda}, \tilde{\xi}_{\Lambda}^{[j]}, \alpha^{[j]}\right) .
$$

The consistency conditions on $S^{[i j]}$ are equivalent to the cocycle conditions on $H^{[i j]}$ at the linearized level. For perturbations around a toric quaternion-Kähler manifold, the variation of the Darboux coordinates and contact potential can be obtained by certain contour integrals of $H^{[i j]}$, as explained in [12], from which the variation of the metric follows. While the integral formulae in [12] are rather involved, they generalize the wellknown Penrose integral formula

$$
\sum_{j} \int_{C_{j}} \frac{\mathrm{d} t}{t} e^{\Phi[j]}(t) H^{[i j]}\left(\xi_{[i]}^{\Lambda}(t), \tilde{\xi}_{\Lambda}^{[j]}(t), \alpha^{[j]}(t)\right)
$$

which maps a holomorphic section of $H^{1}\left(\mathcal{Z}_{\mathcal{M}}, \mathcal{O}(-2)\right)$, which we again represent by a local holomorphic function $H^{[i j]}$, into a function on $\mathcal{M}$ which satisfies a certain set of second order differential equations determined by the quaternion-Kähler structure on $\mathcal{M}$ [10]. In section 5.5, we shall use (5.9) to construct a scalar-valued fivebrane partition function on $\mathcal{M}$.

Finally, we recall that via the moment map construction, continuous isometries of $\mathcal{M}$ are in one-to-one correspondence with real elements of $H^{0}\left(\mathcal{Z}_{\mathcal{M}}, \mathcal{O}(2)\right)$, i.e. with global holomorphic sections of the line bundle $\mathcal{O}(2)$ over $\mathcal{Z}_{\mathcal{M}}$, invariant under the antipodal map. It follows that any continuous isometry of $\mathcal{M}$ can be lifted to a holomorphic isometry of $\mathcal{Z}_{\mathcal{M}}$. Moreover, one can always choose the local Darboux coordinates such that the holomorphic Killing vector is $\partial_{\alpha[i]}$ on each patch $\mathcal{U}_{i}$. In that case, the generating function 
$H^{[i j]}$ becomes independent of $\alpha^{[i]}$, the contact one-form $\mathcal{X}^{[i]}$ becomes globally defined, and $H^{[i j]}$ can be viewed as a generating function for symplectomorphisms with respect to the complex symplectic form $\mathrm{d} \mathcal{X}$ on the quotient of $\mathcal{Z}_{\mathcal{M}}$ by the vector field $\partial_{\alpha^{[i]}}$. Thus, any quaternion-Kähler manifold with a Killing vector can be mapped locally to a hyperkähler manifold of the same dimension. ${ }^{35}$

\subsection{Twistorial description of $\mathcal{M}$}

The twistorial description of the HM moduli space was worked out at tree-level in [10], the one-loop correction was included in $[12,17]$ and D-instantons were incorporated in $[18,19]$. The structure of the D-instanton corrected twistor space is closely related to that of the twistor space of the hyperkähler manifold governing the moduli space of $\mathcal{N}=2$ gauge theories on $\mathbb{R}^{3} \times S^{1}[24]$, in line with the remark at the end of the last subsection.

\subsubsection{Perturbative twistor space}

In the absence of non-perturbative effects, i.e. for the one-loop corrected metric (3.1), the twistor space may be described by three patches $\mathcal{U}_{+}, \mathcal{U}_{-}, \mathcal{U}_{0}$ around the north pole $(t=0)$, south pole $(t=\infty)$ and equator of the $\mathbb{C} P^{1}$ fiber over a fixed point on $\mathcal{M}$. The transition functions from $\mathcal{U}_{0}$ to $\mathcal{U}_{ \pm}$are governed by the prepotential $F$ on $\mathcal{S} \mathcal{K}$, viewed as function of Darboux coordinates $\xi^{\Lambda}$, as explained in [12, 18]. The one-loop correction is incorporated by a non-zero "anomalous dimension", i.e. as a logarithmic singularity around the north and south pole. In the patch $\mathcal{U}_{0}$, the "Darboux coordinates" may be chosen as

$$
\begin{aligned}
\xi^{\Lambda} & =\zeta^{\Lambda}+\frac{\tau_{2}}{2}\left(t^{-1} z^{\Lambda}-t \bar{z}^{\Lambda}\right), \\
\tilde{\xi}_{\Lambda} & =\tilde{\zeta}_{\Lambda}+\frac{\tau_{2}}{2}\left(t^{-1} F_{\Lambda}(z)-t \bar{F}_{\Lambda}(\bar{z})\right), \\
\tilde{\alpha} & =\sigma+\frac{\tau_{2}}{2}\left(t^{-1} W(z)-t \bar{W}(\bar{z})\right)+\frac{i \chi(\mathcal{X})}{24 \pi} \log t,
\end{aligned}
$$

where $W(z) \equiv F_{\Lambda}(z) \zeta^{\Lambda}-z^{\Lambda} \tilde{\zeta}_{\Lambda}$. As in (4.18), we define

$$
\tilde{\xi}_{\Lambda}^{\prime}=\tilde{\xi}_{\Lambda}-A_{\Lambda \Sigma} \xi^{\Sigma}, \quad \alpha^{\prime}=\alpha-\frac{1}{2} A_{\Lambda \Sigma} \xi^{\Lambda} \xi^{\Sigma},
$$

such that, for any charge vector $\gamma \in \Gamma, q_{\Lambda} \xi^{\Lambda}-p^{\Lambda} \tilde{\xi}_{\Lambda}=q_{\Lambda}^{\prime} \xi^{\Lambda}-p^{\Lambda} \tilde{\xi}_{\Lambda}^{\prime}$.

Under monodromies $M$ in $\mathcal{S} \mathcal{K},\left(\xi^{\Lambda}, \tilde{\xi}_{\Lambda}\right)$ transforms linearly under $\rho(M)$. On the other hand, $\tilde{\alpha}$ is shifted by $\kappa(M)$ from (3.22), whereas the middle term is canceling against the variation of the logarithmic term in (5.10) under the R-symmetry rotation $t \mapsto t e^{\mathrm{i} \operatorname{Im} f_{M}} . \operatorname{In}$ particular, under a monodromy $b^{a} \mapsto b^{a}+\epsilon^{a}$ around the large volume point, the Darboux coordinates transform as

$$
\begin{aligned}
& \xi^{0} \mapsto \xi^{0}, \quad \xi^{a} \mapsto \xi^{a}+\epsilon^{a} \xi^{0}, \quad \tilde{\xi}_{a}^{\prime} \mapsto \tilde{\xi}_{a}^{\prime}-\kappa_{a b c} \xi^{b} \epsilon^{c}-\frac{1}{2} \kappa_{a b c} \epsilon^{b} \epsilon^{c} \xi^{0}, \\
& \tilde{\xi}_{0}^{\prime} \mapsto \tilde{\xi}_{0}^{\prime}-\tilde{\xi}_{a}^{\prime} \epsilon^{a}+\frac{1}{2} \kappa_{a b c} \xi^{a} \epsilon^{b} \epsilon^{c}+\frac{1}{6} \kappa_{a b c} \epsilon^{a} \epsilon^{b} \epsilon^{c} \xi^{0}, \quad \tilde{\alpha} \mapsto \tilde{\alpha}+2 \kappa_{a} \epsilon^{a},
\end{aligned}
$$

where we used (5.10), (4.23), and took into account the fact that $\kappa(M)$ must be linear in $\epsilon^{a}, \kappa(M)=\kappa_{a} \epsilon^{a}$. The constants $\kappa_{a}$ should be computable along the lines suggested in the discussion below eq. (3.23), but we do not know their values at this stage.

\footnotetext{
${ }^{35}$ We are grateful to A. Neitzke for discussions on this construction.
} 
Similarly, the Heisenberg action (3.15) lifts to a holomorphic action on $\mathcal{Z}_{\mathcal{M}}$ given by

$$
\begin{aligned}
T_{(H, \kappa)}^{\prime}:\left(\xi^{\Lambda}, \tilde{\xi}_{\Lambda}, \tilde{\alpha}\right) \mapsto & \left(\xi^{\Lambda}+\eta^{\Lambda}, \tilde{\xi}_{\Lambda}+\tilde{\eta}_{\Lambda},\right. \\
& \left.\tilde{\alpha}+2 \kappa-\tilde{\eta}_{\Lambda} \xi^{\Lambda}+\eta^{\Lambda} \tilde{\xi}_{\Lambda}-\left(\eta^{\Lambda} \tilde{\eta}_{\Lambda}-2 \tilde{\eta}_{\Lambda} \theta^{\Lambda}+2 \eta^{\Lambda} \phi_{\Lambda}\right)\right),
\end{aligned}
$$

where $\eta^{\Lambda}, \tilde{\eta}_{\Lambda}, \kappa \in \mathbb{Z}$. Thus, the quotient of $\mathcal{Z}_{\mathcal{M}}$ by translations along $\partial_{\tilde{\alpha}}$ defines a complexified torus $\mathcal{T}_{\mathbb{C}}$, parametrized by the coordinates $\left(\xi^{\Lambda}, \tilde{\xi}_{\Lambda}\right)$ and their complex conjugates, while $e^{\mathrm{i} \pi \tilde{\alpha}}$ parametrizes the fiber of a $\mathbb{C}^{\times}$-bundle $\mathcal{L}_{\Theta}^{\mathbb{C}}$ over $\mathcal{T}_{\mathbb{C}}$. The restriction of $\mathcal{L}_{\Theta}^{\mathbb{C}}$ to the intermediate Jacobian torus $\mathcal{T} \subset \mathcal{T}_{\mathbb{C}}$, at a fixed point on the special Kähler base $\mathcal{S K}$, coincides with the theta line bundle $\mathcal{L}_{\Theta}$. It is interesting to note that the two-form $\mathrm{d} \mathcal{X}$ endows $\mathcal{T}_{\mathbb{C}}$ with a complex symplectic structure, and therefore a natural hyperkähler metric, as explained at the end of section 5.1.

\subsubsection{D-instanton corrected twistor space}

In the presence of D-instantons, the covering of $\mathcal{Z}_{\mathcal{M}}$ must be refined as follows [18, 24]. Over a fixed point in $\mathcal{M}$, the $\mathbb{C} P^{1}$ fiber of $\mathcal{Z}_{\mathcal{M}}$ is divided into angular sectors extending between two BPS rays

$$
\ell_{\gamma}=\left\{t: Z_{\gamma}\left(z^{a}\right) / t \in \mathrm{i} \mathbb{R}^{-}\right\} .
$$

Across such a BPS ray, the Darboux coordinates $\left(\xi^{\Lambda}, \tilde{\xi}_{\Lambda}\right)$ are related by a complex symplectomorphism generated by ${ }^{36}$

$$
H_{\gamma}=\frac{\Omega(\gamma)}{(2 \pi)^{2}} \operatorname{Li}_{2}\left[\sigma_{\mathrm{D}}(\gamma) \mathbf{E}\left(-\Xi_{\gamma}\right)\right]
$$

where $\Xi_{\gamma}=q_{\Lambda} \xi^{\Lambda}-p^{\Lambda} \tilde{\xi}_{\Lambda}$, the factor $\Omega(\gamma)$ is the generalized Donaldson-Thomas (DT) invariant for the charge vector $\gamma, \operatorname{Li}_{2}(x) \equiv \sum_{n=1}^{\infty} x^{n} / n^{2}$ is the dilogarithm function, taking care of multi-covering effects, and $\sigma_{\mathrm{D}}(\gamma)$ is a quadratic refinement (3.17) of the intersection form on the charge lattice $\Gamma$, which plays a crucial role in ensuring consistency across walls of marginal stability [24]. As discussed in section 3.2, although $\sigma_{\mathrm{D}}(\gamma)$ should be related to the quadratic refinement which governs the fivebrane partition function and the NS-axion, and moreover they are expected to coincide, we refrain from identifying them at this point.

The gluing conditions (5.7) for the Darboux coordinates $\xi^{\Lambda}, \tilde{\xi}_{\Lambda}$ can be summarized by the following integral equation

$$
\Xi_{\gamma}=\Theta_{\gamma}+W_{\gamma} / t-\bar{W}_{\gamma} t-\frac{1}{8 \pi^{2}} \sum_{\gamma^{\prime}} \Omega\left(\gamma^{\prime}\right)\left\langle\gamma, \gamma^{\prime}\right\rangle \int_{l_{\gamma^{\prime}}} \frac{\mathrm{d} t^{\prime}}{t^{\prime}} \frac{t+t^{\prime}}{t-t^{\prime}} \operatorname{Li}_{1}\left[\sigma_{\mathrm{D}}\left(\gamma^{\prime}\right) \mathbf{E}\left(-\Xi_{\gamma^{\prime}}\left(t^{\prime}\right)\right)\right]
$$

where $\Theta_{\gamma}=q_{\Lambda} \zeta^{\Lambda}-p^{\Lambda} \tilde{\zeta}_{\Lambda}$, and we recall that $\operatorname{Li}_{1}(x)=-\log (1-x)=\partial_{x} \operatorname{Li}_{2}(x)$. It is worth noting that eq. (5.16) is identical to the integral equation found in [24] in the context of hyperkähler geometry. Moreover, it has the form of a Thermodynamic Bethe Ansatz

\footnotetext{
${ }^{36} \mathrm{Eq}$. (5.14) holds in the one-instanton approximation only, and must be amended to take into account the difference between Darboux coordinates in different angular sectors. The exact generating function was derived [19], and reproduces the symplectomorphism appearing in [24], up to the quadratic refinement $\sigma_{\mathrm{D}}(\gamma)$ which must be added by hand.
} 
equation [24] with an $S$-matrix satisfying all axioms of integrable field theories [100], hinting at a possible integrable structure.

Once the Darboux coordinates $\left(\xi^{\Lambda}, \tilde{\xi}_{\Lambda}\right)$ have been determined, the Darboux coordinate $\tilde{\alpha}$ follows from the contour integral

$$
\begin{aligned}
\tilde{\alpha}= & \sigma+\frac{\tau_{2}}{2}\left(t^{-1} W-t \bar{W}\right)+\frac{\mathrm{i} \chi(\mathcal{X})}{24 \pi} \log t-\frac{1}{4 \pi^{2}} \sum_{\gamma} \Omega(\gamma)\left(t^{-1} W_{\gamma}+t \bar{W}_{\gamma}\right) \mathcal{J}_{\gamma}(0) \\
& +\frac{1}{8 \pi^{2}} \sum_{\gamma} \Omega(\gamma)\left[\frac{\mathrm{i}}{\pi} \int_{\ell_{\gamma}} \frac{\mathrm{d} t^{\prime}}{t^{\prime}} \frac{t+t^{\prime}}{t-t^{\prime}} \operatorname{Li}_{2}\left[\sigma_{\mathrm{D}}(\gamma) \mathbf{E}\left(-\Xi_{\gamma}\left(t^{\prime}\right)\right)\right]+\left(\Theta_{\gamma}+t^{-1} W_{\gamma}-t \bar{W}_{\gamma}\right) \mathcal{J}_{\gamma}(t)\right] \\
& -\frac{1}{64 \pi^{2}} \sum_{\gamma \neq \gamma^{\prime}} \Omega(\gamma) \Omega\left(\gamma^{\prime}\right)\left\langle\gamma, \gamma^{\prime}\right\rangle \int_{\ell_{\gamma}} \frac{\mathrm{d} t^{\prime}}{t^{\prime}} \frac{t+t^{\prime}}{t-t^{\prime}} \operatorname{Li}_{1}\left[\sigma_{\mathrm{D}}(\gamma) \mathbf{E}\left(-\Xi_{\gamma}\left(t^{\prime}\right)\right)\right] \mathcal{J}_{\gamma^{\prime}}\left(t^{\prime}\right),
\end{aligned}
$$

where

$$
\mathcal{J}_{\gamma}(t)=-\int_{\ell_{\gamma}} \frac{\mathrm{d} t^{\prime}}{t^{\prime}} \frac{t+t^{\prime}}{t-t^{\prime}} \operatorname{Li}_{1}\left[\sigma_{\mathrm{D}}(\gamma) \mathbf{E}\left(-\Xi_{\gamma}\left(t^{\prime}\right)\right)\right] .
$$

The contact potential is similarly given by

$$
e^{\Phi}=\frac{\tau_{2}^{2}}{8} e^{-\mathcal{K}(z, \bar{z})}+\frac{\chi(\mathcal{X})}{96 \pi}+\frac{\mathrm{i}}{16 \pi^{2}} \sum_{\gamma} \Omega(\gamma) \int_{\ell_{\gamma}} \frac{\mathrm{d} t}{t}\left(t^{-1} W_{\gamma}-t \bar{W}_{\gamma}\right) \operatorname{Li}_{1}\left[\sigma_{\mathrm{D}}(\gamma) \mathbf{E}\left(-\Xi_{\gamma}(t)\right)\right] .
$$

Note that it is constant on $\mathbb{C} P^{1}$, as required by the continuous shift isometry along $\sigma$, and may be chosen to define the "instanton corrected 4D string coupling" $e^{\phi}=e^{\Phi}$.

Eqs. (5.16) (and subsequently (5.17), (5.19)) may be solved recursively by first substituting $\Xi_{\gamma}(t)$ on the r.h.s. by its perturbative value $\Xi_{\gamma}^{(0)}(t)=\Theta_{\gamma}+W_{\gamma} / t-\bar{W}_{\gamma} t$, computing the integral to obtain the one-instanton correction $\Xi_{\gamma}^{(1)}$ and iterating this procedure. This provides an asymptotic series for the exact, D-instanton corrected Darboux coordinates,

$$
\Xi_{\gamma}(t)=\Theta_{\gamma}+W_{\gamma} / t-\bar{W}_{\gamma} t+\sum_{N=1}^{\infty} \Xi_{\gamma}^{(N)}
$$

where $\Xi_{\gamma}^{(N)}$ involves $N$ nested contour integrals of sums of products of $N$ DT-invariants $\Omega\left(\gamma_{k}\right), k=1, \ldots, N$. This can be interpreted as corrections from multi-centered instanton configurations, with $\Omega\left(\gamma_{k}\right)$ providing the contribution to the instanton measure of the $k$ th center. In the vector multiplet context, these are instead multi-centered Euclidean black holes whose worldline winds around the Euclidean time direction, and $\Omega\left(\gamma_{k}\right)$ is instead the indexed degeneracy of the $k$ th center. At fixed $N$, the sum over $\gamma_{k}$ typically has zero radius of convergence, due to the expected exponential growth of the generalized DT-invariants. It was argued in [27] that the ambiguity of this asymptotic series is of the same order as the corrections expected from NS5-branes.

Noting that the BPS rays $\ell_{\gamma}$ and $\ell_{n \gamma}$ are identical, one may dispose of the dilogarithm in above formulas by replacing the integer-valued DT invariants $\Omega(\gamma)$ by the rational-valued DT invariants ${ }^{37}$

$$
\bar{\Omega}(\gamma)=\sum_{d \mid \gamma} \frac{1}{d^{2}} \Omega(\gamma / d), \quad \Omega(\gamma)=\sum_{d \mid \gamma} \frac{1}{d^{2}} \mu(d) \bar{\Omega}(\gamma / d),
$$

\footnotetext{
${ }^{37}$ The rational invariants $\bar{\Omega}(\gamma)$ are also relevant for S-duality and wall-crossing [101, 102].
} 
where $\mu(d)$ is the Möbius function. In particular, one finds that the D-instanton corrected twistor space can be equally obtained from the above construction where the holomorphic functions (5.15) are replaced by

$$
\tilde{H}_{\gamma}=\frac{\sigma_{\mathrm{D}}(\gamma)}{(2 \pi)^{2}} \bar{\Omega}(\gamma) \mathbf{E}\left(p^{\Lambda} \tilde{\xi}_{\Lambda}^{\prime}-q_{\Lambda}^{\prime} \xi^{\Lambda}\right)
$$

Here we expressed the result in terms of the primed charges $q_{\Lambda}^{\prime}$ and Darboux coordinates $\tilde{\xi}_{\Lambda}^{\prime}$ and used the fact that $\sigma_{\mathrm{D}}(d \gamma)=\left(\sigma_{\mathrm{D}}(\gamma)\right)^{d}$ for any positive integer $d$. As an illustration, the contact potential in the one-instanton approximation is found to be [18]

$$
e^{\Phi}=\frac{\tau_{2}^{2}}{8} e^{-\mathcal{K}(z, \bar{z})}+\frac{\chi(\mathcal{X})}{96 \pi}+\frac{1}{4 \pi^{2}} \sum_{\gamma \in \Gamma} \sigma_{\mathrm{D}}(\gamma) \bar{\Omega}(\gamma)\left|W_{\gamma}\right| \cos \left(2 \pi \Theta_{\gamma}\right) K_{1}\left(4 \pi\left|W_{\gamma}\right|\right)+\ldots
$$

In this approximation, $e^{\Phi}$ will in general be discontinuous across lines of marginal stability in $\mathcal{S K}$, where $\bar{\Omega}(\gamma)$ may jump. However, this discontinuity is canceled by the two- and higher-instanton contributions, provided the jump in $\bar{\Omega}(\gamma)$ obeys the Kontsevich-Soibelman wall-crossing formula [24].

\subsubsection{S-duality in twistor space}

As mentioned in section 4.1 , in the infinite volume and zero coupling limit, $\mathcal{Q}_{K}(\hat{\mathcal{X}})$ admits an isometric action of $\mathrm{SL}(2, \mathbb{R})$ given in $(4.31)$. This action lifts to a holomorphic action on $\mathcal{Z}_{\mathcal{M}}$, given in terms of the Darboux coordinates in the patch $\mathcal{U}_{0}$ as [18]

$$
\begin{gathered}
\xi^{0} \mapsto \frac{a \xi^{0}+b}{c \xi^{0}+d}, \quad \xi^{a} \mapsto \frac{\xi^{a}}{c \xi^{0}+d}, \quad \tilde{\xi}_{a}^{\prime} \mapsto \tilde{\xi}_{a}^{\prime}+\frac{c}{2\left(c \xi^{0}+d\right)} \kappa_{a b c} \xi^{b} \xi^{c}-c_{2, a} \varepsilon(\delta), \\
\left(\begin{array}{c}
\tilde{\xi}_{0}^{\prime} \\
\alpha^{\prime}
\end{array}\right) \mapsto\left(\begin{array}{cc}
d-c \\
-b & a
\end{array}\right)\left(\begin{array}{c}
\tilde{\xi}_{0}^{\prime} \\
\alpha^{\prime}
\end{array}\right)+\frac{1}{6} \kappa_{a b c} \xi^{a} \xi^{b} \xi^{c}\left(\begin{array}{c}
c^{2} /\left(c \xi^{0}+d\right) \\
-\left[c^{2}\left(a \xi^{0}+b\right)+2 c\right] /\left(c \xi^{0}+d\right)^{2}
\end{array}\right) .
\end{gathered}
$$

Indeed, substituting the Darboux coordinates (5.10) in (5.24) and using the classical mirror map (4.29), one recovers the isometric action (4.31), supplemented with the following SU(2) action on the $\mathbb{C} P^{1}$ fiber,

$$
t \mapsto \frac{c \tau_{2}+t\left(c \tau_{1}+d\right)+t|c \tau+d|}{\left(c \tau_{1}+d\right)+|c \tau+d|-t c \tau_{2}}
$$

Under the holomorphic action (5.24), the complex contact one-form transforms by an overall holomorphic factor $\mathcal{X}^{[i]} \rightarrow \mathcal{X}^{[i]} /\left(c \xi^{0}+d\right)$, leaving the complex contact structure invariant. Moreover, the relations

$$
e^{\Phi} \mapsto \frac{e^{\Phi}}{|c \tau+d|}, \quad \frac{|t|}{\left(1+|t|^{2}\right)^{2}} \mapsto \frac{|t|}{\left(1+|t|^{2}\right)^{2}} \frac{\left|c \xi^{0}+d\right|^{2}}{|c \tau+d|^{2}}
$$

ensure that the Kähler potential varies by a Kähler transformation, consistent with the rescaling of $\mathcal{X}^{[i]}$,

$$
K_{\mathcal{Z}} \mapsto K_{\mathcal{Z}}-\log \left(\left|c \xi^{0}+d\right|\right)
$$


Note that $e^{\Phi}$ transforms like $\tau_{2}^{1 / 2}$, i.e. has modular weight $\left(-\frac{1}{2},-\frac{1}{2}\right)$. The character $c_{2, a} \varepsilon(\delta)$ in the transformation of $\tilde{\xi}_{a}^{\prime}$ follows from the shift of the real coordinate $c_{a}$ in (4.31). It may be interpreted geometrically by saying that $\mathbf{E}\left(p^{a} \tilde{\xi}_{a}^{\prime}\right)$ transforms like the automorphy factor of a multi-variable Jacobi form of index $m_{a b}=\frac{1}{2} \kappa_{a b c} p^{c}$ and with multiplier system $\mathbf{E}\left(-c_{2 a} p^{a} \varepsilon(\delta)\right)$.

As discussed in section 4.1, worldsheet instantons and the one-loop correction break continuous S-duality, but an $\mathrm{SL}(2, \mathbb{Z})$ subgroup can be preserved upon including D1-D(1)-brane instantons, as shown in [21]. The corrections to the Darboux coordinates from such instanton configurations were computed in [20]. It was found that the Darboux coordinates continue to transform under the tree-level transformation rules (5.24), up to a local contact transformation. It is possible to construct new Darboux coordinates which transform precisely according to (5.24) (those were called $\xi^{\left[0_{B}\right]}$ in [20]), although these coordinates will only be valid in a certain open set in $\mathbb{C} P^{1}$. If S-duality is indeed maintained at the quantum level, this should remain true in the presence of D3-brane instantons, and ultimately, in the presence of D5 and NS5-brane instantons. In the following, we shall be using such adapted Darboux coordinates, though their explicit construction in the presence of D3-D1-D(-1) instantons is still an open problem [103].

\subsection{Contact structure for fivebrane instantons}

In this section, we use the qualitative insights gained in the previous sections and the twistor techniques reviewed above to determine the form of NS5-brane instanton corrections to the HM moduli space $\mathcal{M}$, consistently with supersymmetry and S-duality. Our aim is to determine the data governing the deformation of the complex contact structure on $\mathcal{Z}_{\mathcal{M}}$ which encode the NS5-brane corrections. Such data are provided by contours $\ell_{k, p, \hat{\gamma}}$ and the associated holomorphic sections $H_{k, p, \hat{\gamma}} \in H^{1}\left(\mathcal{Z}_{\text {pert }}, \mathcal{O}(2)\right)$ describing discontinuities of complex Darboux coordinates across these contours. To find them in the one-instanton approximation, we covariantize under S-duality the data responsible for the D-instanton corrections, which are given by the holomorphic sections (5.22) and the contours $\ell_{\gamma}$ (5.14). Performing this covariantization, we assume that S-duality requires the invariance of all these data, namely, that every pair $\left(H_{k, p, \hat{\gamma}}, \ell_{k, p, \hat{\gamma}}\right)$ is mapped to another one so that the total structure of the twistor space remains intact. As apparent from the analysis of the D1-D(-1)-instanton sector [20], this assumption is strictly speaking unwarranted, since Sduality can produce contributions which can be canceled by local contact transformations. Moreover, it leads to a dense set of mutually intersecting discontinuities on the $\mathbb{C} P^{1}$ fiber, whose mathematical status is questionable. However, we shall see that it does lead to an appealing result which matches general expectations about the fivebrane contributions. We therefore believe that the main features of our approach should subsist in a more sophisticated treatment.

Thus, we start with the holomorphic sections $\tilde{H}_{\gamma} \in H^{1}\left(\mathcal{Z}_{\text {pert }}, \mathcal{O}(2)\right)$ given in (5.22), which describe the D-instanton corrections to the perturbative metric (3.1) in the oneinstanton approximation. In the absence of D5-brane instanton corrections $p^{0}=0$, and for a fixed D3-brane charge $p^{a}$, the corrections to the moduli space metric from $\tilde{H}_{\gamma}$ should 
preserve the isometric action of $\mathrm{SL}(2, \mathbb{Z})$ (or a finite index subgroup thereof). On the other hand, the instanton corrections with $p^{0} \neq 0$ break S-duality completely, unless they are supplemented by additional contributions from NS5-branes, or more generally $(p, k) 5$ branes. It is natural to propose that S-duality is restored by refining the contact structure on $\mathcal{Z}_{\mathcal{M}}$ by adding all images of $\left(\tilde{H}_{\gamma}, \ell_{\gamma}\right)$ under $\Gamma_{\infty} \backslash \mathrm{SL}(2, \mathbb{Z})$, where $\Gamma_{\infty}$ is the subgroup of strictly upper triangular matrices, to the data describing discontinuities of Darboux coordinates. The coset $\Gamma_{\infty} \backslash \mathrm{SL}(2, \mathbb{Z})$ can be parametrized by matrices

$$
\delta=\left(\begin{array}{ll}
a & b \\
c & d
\end{array}\right)
$$

where $(c, d)$ run over coprime integers, and the integers $(a, b)$ are functions of $(c, d)$, determined up to multiples of $(c, d)$ by the condition $a d-b c=1$. Thus, we propose that in the one-instanton approximation the contact structure is described by the following data

$$
H_{k, p, \hat{\gamma}}=\delta \cdot \tilde{H}_{\gamma}, \quad \ell_{k, p, \hat{\gamma}}=\delta \cdot \ell_{\gamma},
$$

where $\delta \cdot \tilde{H}_{\gamma}$ and $\delta \cdot \ell_{\gamma}$ denotes the image of (5.22) and (5.14) under the $\operatorname{SL}(2, \mathbb{Z})$ transformation (5.28) with $(c, d)=\left(-k / p^{0}, p / p^{0}\right)$ as in (4.39), and we recall that $\hat{\gamma}=\left(p^{a}, \hat{q}_{a}, \hat{q}_{0}\right)$.

We expect that $H_{k, p, \hat{\gamma}}$ represents the effect on the contact structure of a $(p, k) 5$-brane. Using (5.24), it evaluates to

$$
H_{k, p, \hat{\gamma}}=\frac{\sigma_{\mathrm{D}}(\gamma)}{4 \pi^{2}} \bar{\Omega}(\gamma) \mathbf{E}\left(-\frac{k}{2} S_{\alpha}+\frac{p^{0}\left(k \hat{q}_{a}\left(\xi^{a}-n^{a}\right)+p^{0} \hat{q}_{0}\right)}{k^{2}\left(\xi^{0}-n^{0}\right)}+a \frac{p^{0} q_{0}^{\prime}}{k}-c_{2, a} p^{a} \varepsilon(\delta)\right)
$$

where

$$
S_{\alpha} \equiv \tilde{\alpha}+\left(\xi^{\Lambda}-2 n^{\Lambda}\right) \tilde{\xi}_{\Lambda}^{\prime}+2 \frac{N\left(\xi^{a}-n^{a}\right)}{\xi^{0}-n^{0}}
$$

$p^{0}$ is defined as $\operatorname{gcd}(p, k), \gamma=\left(p^{0}, p^{a}, q_{a}, q_{0}\right) \in \Gamma$, and we have denoted $n^{0} \equiv p / k, n^{a} \equiv p^{a} / k$, valued in $\mathbb{Z} / k$. On the other hand, the transformation (5.25) can be used to find the contour $\ell_{k, p, \hat{\gamma}}$. It is easily seen to be a half-circle stretching between the two zeros of $\xi^{0}-n^{0}$, which, as will become clear below in (5.44), are the only singularities of $H_{k, p, \hat{\gamma}}$. The direction of the contour is determined by the same condition as (5.14) with $Z_{\gamma}$ replaced by $W_{k, p, \hat{\gamma}}$, which was introduced in (4.40). As a result, the complete set of contours is given by an infinite number of copies of the "melon-shaped" picture for D-instantons [18, 24], rotated into each other by $\operatorname{SL}(2, \mathbb{Z})$ transformations. For fixed $(p, k)$, the new BPS rays extend between two antipodal points whose location is completely determined by a sole rational number $p / k$.

A crucial requirement on the new contact structure is that it should be invariant under the Heisenberg group (5.13). The invariance with respect to $T_{(0, \tilde{\eta}), \kappa}^{\prime}$ follows trivially from the invariance of the holomorphic functions (5.30), provided the fivebrane characteristics $\theta^{\Lambda}$ are set to zero. In contrast, $H_{k, p, \hat{\gamma}}$ are not individually invariant under $T_{(\eta, 0), 0}^{\prime}$. To be nevertheless consistent with the Heisenberg symmetry, the set of functions (5.30) should be globally invariant under $T_{(\eta, 0), 0}^{\prime}$, i.e. $H_{k, p, \hat{\gamma}}$ should be mapped to $H_{k, p^{\prime}, \hat{\gamma}^{\prime}}$ for a suitably chosen map $(p, \hat{\gamma}) \mapsto\left(p^{\prime}, \hat{\gamma}^{\prime}\right)$, with the contours $\ell_{k, p, \hat{\gamma}}$ following the same pattern. 
As we demonstrate in appendix A, under the crucial assumption that $\bar{\Omega}(\gamma)$ are invariant under the spectral flow (4.24), the combined action of the Heisenberg symmetry together with a shift $p \mapsto p+k \eta^{0}$ and the spectral flow action (4.24) with parameter $\epsilon^{a}=k \eta^{a} / p^{0}$ on the charges $p^{a}, q_{a}, q_{0}$ leaves the set of functions (5.30) globally invariant, up to a phase factor $\nu(\eta)$ computed in (A.6),

$$
T_{\left(\eta^{\Lambda}, 0,0\right)}^{\prime} \cdot H_{k, p+k \eta^{0}, \hat{\gamma}\left[k \eta^{a} / p^{0}\right]}=\nu(\eta) H_{k, p, \hat{\gamma}}
$$

where $\hat{\gamma}[\epsilon]=\left(p^{a}+\epsilon^{a}, \hat{q}_{a}, \hat{q}_{0}\right)$. Moreover, the contours $\ell_{k, p, \hat{\gamma}}$ stay invariant under this combined transformation. Indeed, their endpoints, being the zeros of $\xi^{0}-n^{0}$ are explicitly invariant and the direction of approach is also conserved by virtue of the invariance of $W_{k, p, \hat{\gamma}}(4.40)$. Under the same assumptions, $H_{k, p, \hat{\gamma}}$ are also invariant, up to a phase factor $\nu^{\prime}(\eta)$ computed in (A.12), under the combined action of monodromies (5.12) and of the spectral flow (4.24) with parameter $\epsilon^{\prime a} \equiv\left(p / p^{0}\right) \epsilon^{a}$,

$$
M_{\epsilon^{a}} \cdot H_{k, p, \hat{\gamma}\left[p \epsilon / p^{0}\right]}=\nu^{\prime}(\epsilon) H_{k, p, \hat{\gamma}}
$$

where $M_{\epsilon^{a}}$ is the monodromy operator acting on complex Darboux coordinates as in (5.12).

Unfortunately, while the phase $\nu(\eta)$ can be set to one in the $k=1$ case by an ad hoc choice of the characteristics, namely $\theta_{\mathrm{D}}^{\Lambda}=0, \phi_{0}=A_{00} / 2, \phi_{a}-\phi_{\mathrm{D}, a}=A_{a a}$, this is not possible for general values of $k$, and it does not lead to a cancelation of the phase $\nu^{\prime}(\epsilon)$. Moreover, these conditions are not compatible with the transformation rule (2.24) of the characteristics under monodromies, and therefore should probably not be taken seriously. This tension between S-duality, Heisenberg and monodromy invariance indicates that it may be necessary to relax some of our assumptions about the way these symmetries are realized. In this paper, we shall ignore this difficulty and proceed with the analysis as though the phases $\nu(\eta)$ and $\nu^{\prime}(\epsilon)$ were absent, leaving a more complete analysis for future work.

\subsection{Poincaré series for NS5-instantons and the topological string amplitude}

It is instructive to construct a formal section of $H^{1}\left(\mathcal{Z}_{\text {pert }}, \mathcal{O}(2)\right)$ given by a sum of the holomorphic functions (5.30) over the integer charges $p, p^{a}$ and $q_{\Lambda}$ :

$$
H_{\mathrm{NS} 5}^{(k)}(\xi, \tilde{\xi}, \tilde{\alpha})=\sum_{p, p^{a}, q_{\Lambda}} H_{k, p, \hat{\gamma}}(\xi, \tilde{\xi}, \tilde{\alpha}) .
$$

This sum is formal since each term is attached to a different contour on $\mathbb{C} P^{1}$. Nevertheless, it can be meaningfully inserted into general formulas for Darboux coordinates and the contact potential from [12], provided the sum is performed after integration along $\mathbb{C} P^{1}$.

Let us now recast (5.34) in the form

$$
H_{\mathrm{NS} 5}^{(k)}(\xi, \tilde{\xi}, \tilde{\alpha})=\frac{1}{4 \pi^{2}} \sum_{\substack{\mu \in\left(\Gamma_{m} /|k|\right) / \Gamma_{m} \\ n \in \Gamma_{m}+\mu+\theta}} H_{\mathrm{NS} 5}^{(k, \mu)}\left(\xi^{\Lambda}, n^{\Lambda}\right) \mathbf{E}\left(k n^{\Lambda}\left(\tilde{\xi}_{\Lambda}-\phi_{\Lambda}\right)-\frac{k}{2}\left(\tilde{\alpha}+\xi^{\Lambda} \tilde{\xi}_{\Lambda}\right)\right)
$$

Using results of the previous subsection, we find that the characteristics $\theta^{\Lambda}$ vanish and that the function $H_{\mathrm{NS} 5}^{(k, \mu)}\left(\xi^{\Lambda}, n^{\Lambda}\right)$ is invariant, up to the phase (A.6), under simultaneous 
shift of $\xi^{\Lambda}$ and $n^{\Lambda}$. Therefore, up to the same phase, it is actually a function of only their difference,

$$
H_{\mathrm{NS} 5}^{(k, \mu)}\left(\xi^{\Lambda}, n^{\Lambda}\right)=\nu\left(n^{\Lambda}-\mu^{\Lambda}\right) H_{\mathrm{NS} 5}^{(k, \mu)}\left(\xi^{\Lambda}-n^{\Lambda}\right),
$$

where the function on the right is given by

$$
H_{\mathrm{NS} 5}^{(k, \mu)}\left(\xi^{\Lambda}\right)=\sum_{q_{\Lambda}} K_{k, \mu^{\Lambda}, q_{\Lambda}} \bar{\Omega}(\gamma) \mathbf{E}\left(-\frac{k N\left(\xi^{a}\right)}{\xi^{0}}+\frac{p^{0}\left(k \hat{q}_{a} \xi^{a}+p^{0} \hat{q}_{0}\right)}{k^{2} \xi^{0}}+\frac{k}{2} A_{\Lambda \Sigma} \xi^{\Lambda} \xi^{\Sigma}\right),
$$

and we collected various constant factors into

$$
K_{k, \mu^{\Lambda}, q_{\Lambda}}=\sigma_{\mathrm{D}}(\gamma) \mathbf{E}\left(-\frac{k}{2} A_{\Lambda \Sigma} \mu^{\Lambda} \mu^{\Sigma}+a \frac{p^{0} q_{0}^{\prime}}{k}-k c_{2, a} \mu^{a} \varepsilon(\delta)+k \mu^{\Lambda} \phi_{\Lambda}\right) .
$$

In this expression, $\gamma=\left(p^{0}, k \mu^{a}, q_{a}, q_{0}\right)$ where $p^{0}=\operatorname{gcd}\left(k, k \mu^{0}\right)$,

$$
\hat{q}_{a}=q_{a}^{\prime}+\frac{k^{2}}{2 p^{0}} \kappa_{a b c} \mu^{b} \mu^{c}, \quad \hat{q}_{0}=q_{0}^{\prime}+\frac{k}{p^{0}} \mu^{a} q_{a}^{\prime}+\frac{k^{3}}{3\left(p^{0}\right)^{2}} \kappa_{a b c} \mu^{a} \mu^{b} \mu^{c},
$$

and $q_{a}^{\prime}, q_{0}^{\prime}$ are related to $q_{a}, q_{0}$ via (4.9). The vectors $k\left(\mu^{0}, \mu^{a}\right)$ are identified as the residue class of $\left(p, p^{a}\right)$ modulo $k$. Setting the phase factor $\nu(n-\mu)$ in (5.36) to one (which can be achieved in the case $k=1$ by the aforementioned ad hoc choice of characteristics, and should result more generally from a fully consistent treatment of Heisenberg, S-duality and monodromy symmetries), we find that the holomorphic section $H_{\mathrm{NS} 5}^{(k)}(5.35)$ encoding the fivebrane corrections has the form of a (non-Gaussian) theta series.

Having recast the fivebrane corrections (5.34) into the form (5.35), we can now unravel the connection between the fivebrane wave-function $H_{\mathrm{NS} 5}^{(1)}$ for $k=1$ and the A-model topological string amplitude discussed in section 2.5. To this end, notice that for $k=1$, eq. (5.37) simplifies dramatically to

$$
H_{\mathrm{NS} 5}^{(1,0)}\left(\xi^{\Lambda}\right)=\epsilon \sum_{\hat{q}_{a}, \hat{q}_{0}} \bar{\Omega}(\gamma)(-1)^{\hat{q}_{0}} \mathbf{E}\left(-\frac{N\left(\xi^{a}\right)}{\xi^{0}}+\frac{\hat{q}_{a} \xi^{a}+\hat{q}_{0}}{\xi^{0}}+\frac{1}{2} A_{\Lambda \Sigma} \xi^{\Lambda} \xi^{\Sigma}\right) .
$$

Here we fixed the characteristics as in eqs. (A.7), (A.9) as required for Heisenberg invariance and took into account that $p^{0}=1, \mu^{\Lambda}=0$ for $k=1$. The factor $\epsilon=(-1)^{A_{00}-\phi_{\mathrm{D}, 0} / 2}$ is irrelevant and will be omitted in what follows. Now recall the relation (2.84) which expresses the A-model wave function $e^{F_{\mathrm{hol}}(z, \lambda)}$ in terms of the DT partition function. Identifying $\left(Q_{a}, 2 J\right)=\left(\hat{q}_{a}+c_{2, a} / 24, \hat{q}_{0}\right)$ as in (4.28) (which are integer-valued when $p^{0}=1$, as noted below (4.28)) and using the relation ${ }^{38}(2.77)$ between $F_{\text {hol }}(z, \lambda)$ and the wave function $\Psi_{\mathbb{R}}^{\text {top }}\left(\xi^{\Lambda}\right)$, we obtain the twistor space version of the real-polarized A-model topological string wave function

$$
\begin{aligned}
\Psi_{\mathbb{R}}^{\mathrm{top}}\left(\xi^{\Lambda}\right)= & \left(\xi^{0}\right)^{1+\left(1+\epsilon_{\mathrm{GW}}\right) \frac{\chi(\hat{\mathcal{X}})}{24}}\left[M\left(e^{2 \pi \mathrm{i} / \xi^{0}}\right)\right]^{\left(\frac{1}{2}-\epsilon_{\mathrm{DT}}\right) \chi(\hat{\mathcal{X}})} \sum_{\hat{q}_{0}, \hat{q}_{a}}(-1)^{\hat{q}_{0}} N_{D T}\left(\hat{q}_{a}+\frac{1}{24} c_{2 a}, \hat{q}_{0}\right) \\
& \times \mathbf{E}\left(-\frac{N\left(\xi^{a}\right)}{\xi^{0}}+\frac{1}{2} A_{\Lambda \Sigma} \xi^{\Lambda} \xi^{\Sigma}+\frac{\hat{q}_{a} \xi^{a}}{\xi^{0}}+\frac{\hat{q}_{0}}{\xi^{0}}\right) .
\end{aligned}
$$

\footnotetext{
${ }^{38}$ Here we use the relation $\lambda=2 \pi /\left(\mathrm{i} \xi^{0}\right)$, rather than $\lambda=1 /\left(\xi^{0} \sqrt{2 \pi}\right)$ as stated in $(2.77)$. We do not understand the origin of this normalization mismatch. Note also that the prefactor in (5.41) behaves as $\left(\xi^{0}\right)^{1+\left(2 \epsilon_{\mathrm{DT}}+\epsilon_{\mathrm{GW}}\right) \frac{\chi(\hat{\mathcal{X}})}{24}}$, which seems unnatural for the usual choices of $\epsilon_{\mathrm{GW}}, \epsilon_{\mathrm{DT}}$ given in the literature.
} 
Comparing (5.41) with (5.40) and identifying the DT-invariants $N_{D T}\left(\hat{q}_{a}+\frac{1}{24} c_{2 a}, \hat{q}_{0}\right)$ with the rational instanton measure $\bar{\Omega}(\gamma)$, we find that the wave function $H_{\mathrm{NS} 5}^{(1,0)}\left(\xi^{\Lambda}\right)$ governing NS5-brane instantons in type IIB/ $\hat{\mathcal{X}}$ is proportional to the wave function of the topological A-model on $\hat{\mathcal{X}}$ in the real polarization,

$$
H_{\mathrm{NS} 5}^{(1,0)}\left(\xi^{\Lambda}\right)=\left(\xi^{0}\right)^{-1-\left(1+\epsilon_{\mathrm{GW}}\right) \frac{\chi(\hat{\mathcal{X}})}{24}}\left[M\left(e^{2 \pi \mathrm{i} / \xi^{0}}\right)\right]^{\left(\epsilon_{\mathrm{DT}}-\frac{1}{2}\right) \chi(\hat{\mathcal{X}})} \Psi_{\mathbb{R}}^{\mathrm{top}}\left(\xi^{\Lambda}\right) .
$$

We note that the sign factor $(-1)^{2 J}=e^{\pi i \hat{q}_{0}}$ predicted by the GW/DT relation nicely agrees with the factor $\mathbf{E}\left(-\frac{1}{2} q_{\Lambda} p^{\Lambda}\right)$ in the quadratic refinement $\sigma_{\Theta}$. The relation (5.42) is moreover generally consistent with the fact that both $H_{\mathrm{NS} 5}^{(1,0)}\left(\xi^{\Lambda}\right)$ and $\Psi_{\mathbb{R}}^{\mathrm{top}}\left(\xi^{\Lambda}\right)$ should transform under monodromies according to the metaplectic representation, although the powers of $\xi^{0}$ and $M\left(e^{2 \pi \mathrm{i} / \xi^{0}}\right)$ appear to spoil these transformation properties. Eq. (5.42) also suggests that the proper mathematical interpretation of the real-polarized wave function $\Psi_{\mathbb{R}}^{\text {top }}$ may be as a section of $H^{1}\left(\mathcal{Z}_{\mathcal{M}}\right)$, rather than $H^{0}\left(\mathcal{M}_{K}\right)$ as is commonly assumed.

By mirror symmetry, (5.42) should also determine the wave function governing NS5brane corrections in type IIA string theory compactified on $\mathcal{X}$, in terms of the wave function $\Psi_{\mathbb{R}}^{\text {top }}$ of the topological B-model on $\mathcal{X}$ in the real polarization, in agreement with general expectations expressed in [59], and earlier in [32, 104]. We offer further support for this assertion in the remainder of this section. More generally, we expect that the wave function $H_{\mathrm{NS} 5}^{(k, \mu)}\left(\xi^{\Lambda}\right)$ for $k>1$ in type IIA should originate from a higher rank version of the topological B-model on $\mathcal{X}$, presumably related to the generalized invariants of Joyce and Song [26].

\subsection{Fivebrane partition function from twistor space}

Having identified candidate sections $H_{k, p, \hat{\gamma}}$ in $H^{1}\left(\mathcal{Z}_{\mathcal{M}}, \mathcal{O}(2)\right)$ governing the corrections to the contact structure on the twistor space $\mathcal{Z}_{\mathcal{M}}$ from $k$ fivebranes, we would now like to make contact with the qualitative discussion of fivebrane instanton corrections to the HM moduli space metric of sections 3 and 4 . To this end, we should in principle evaluate the corresponding corrections to the Darboux coordinates and contact potential $\Phi$ using the integral formulae in $[12,18]$, and from them obtain the corrections to the metric on $\mathcal{M}$. This analysis is however beyond the scope of the present work.

Instead, we shall address the simpler question raised in section 1.6, namely construct a scalar-valued function on $\mathcal{M}$ which generalizes the Gaussian flux partition function $\mathcal{Z}_{\mathrm{G}}^{(k)}$ at finite coupling. To this end, let us view (5.34) as a formal holomorphic section of $H^{1}\left(\mathcal{Z}_{\mathcal{M}}, \mathcal{O}(-2)\right)$ (barring global issues) and apply the standard Penrose transform (5.9). This produces a certain function $\mathcal{Z}_{\mathrm{NS} 5}^{(k)}$ on the perturbative HM moduli space, satisfying certain second order differential equations. We shall see that this function reduces to a close variant of the Gaussian flux partition function $\mathcal{Z}_{\mathrm{G}}^{(k)}$ discussed in section 2 in the limit $g_{s}=0$, thereby motivating the name "non-Gaussian NS5-brane partition function". This computation may also be viewed as a warm-up for the more complicated computations involved in extracting the corrections to the HM metric from the $H^{1}\left(\mathcal{Z}_{\mathcal{M}}, \mathcal{O}(2)\right)$ section (5.34). 


\subsubsection{Penrose transform}

We thus consider the Penrose transform (5.9) of a single term $H_{k, p, \hat{\gamma}}$ in the sum (5.34), given in (5.30):

$$
\int_{\ell_{k, p, \hat{\gamma}}} \frac{\mathrm{d} t}{t} \mathbf{E}\left(-\frac{k}{2}\left(\tilde{\alpha}+\left(\xi^{\Lambda}-2 n^{\Lambda}\right) \tilde{\xi}_{\Lambda}^{\prime}\right)-\frac{k N\left(\xi^{a}-n^{a}\right)}{\xi^{0}-n^{0}}+\frac{k p^{0} \hat{q}_{a}\left(\xi^{a}-n^{a}\right)+\left(p^{0}\right)^{2} \hat{q}_{0}}{k^{2}\left(\xi^{0}-n^{0}\right)}\right),
$$

where the contour $\ell_{k, p, \hat{\gamma}}$ interpolates between the two zeros of $\xi^{0}-n^{0}$, and passes through the saddle point to be analyzed below. We omitted the contact potential which appears as the overall factor since in our approximation it is independent of $t$ and also dropped the constant factors given by the quadratic refinement and the last two terms in (5.30). Note that, by construction, (5.43) is proportional to $e^{-\mathrm{i} \pi k \sigma}$, and manifestly invariant under Kähler transformations and (after summing over charges, ignoring the phases discussed in appendix A) under Heisenberg translations.

Using the expression for the Darboux coordinates given in (5.10), the integrand evaluates to

$$
t^{-\frac{\chi(\hat{\mathcal{X}})}{24}} \mathbf{E}\left(-k\left[\frac{1}{2}\left(\sigma+\left(\zeta^{\Lambda}-2 n^{\Lambda}\right) \tilde{\zeta}_{\Lambda}^{\prime}\right)-\frac{\tau_{2}^{2}}{4} \operatorname{Re}\left(\bar{z}^{\Lambda} F_{\Lambda}^{\prime}\right)+\frac{\mathcal{B}+\frac{\tau_{2}}{2}\left(t^{-1} \mathcal{A}-t \overline{\mathcal{A}}\right)}{\tilde{\tau}_{1}+\frac{\tau_{2}}{2}\left(t^{-1}-t\right)}\right]\right)
$$

where we abbreviated $\tilde{\tau}=\tau-n^{0}$ and introduced

$$
\begin{aligned}
& \mathcal{A}=\frac{\tau_{2}^{2}}{4} \bar{z}^{\Lambda} F_{\Lambda}^{\prime}-\frac{1}{2}\left(\zeta^{\Lambda}-n^{\Lambda}\right)\left(\zeta^{\Sigma}-n^{\Sigma}\right) F_{\Lambda \Sigma}^{\prime}-\frac{p^{0} \hat{q}_{a}}{k^{2}} z^{a}, \\
& \mathcal{B}=N(\zeta-n)-\frac{\tau_{2}^{2}}{2}\left(\tilde{\tau}_{1} \operatorname{Re} N(z)+\left(\zeta^{a}-n^{a}\right) \kappa_{a b c} t^{b} t^{c}\right)-\frac{p^{0} \hat{q}_{a}}{k^{2}}\left(\zeta^{a}-n^{a}\right)-\frac{\left(p^{0}\right)^{2} \hat{q}_{0}}{k^{3}} .
\end{aligned}
$$

It is useful to note that

$$
\frac{k \tau_{2}}{2|\tilde{\tau}|^{2}}\left(\tilde{\tau}_{1} \operatorname{Re} \mathcal{A}-\mathcal{B}-\mathrm{i}|\tilde{\tau}| \operatorname{Im} \mathcal{A}\right)=W_{k, p, \hat{\gamma}}
$$

reproduces the quantity introduced in (4.40). The integral over $t$ is dominated in the weak coupling limit by a saddle point at

$$
t_{s}=\mathrm{i} \frac{-\tau_{2} \operatorname{Im} \mathcal{A}-\sqrt{\left(\mathcal{B}-\tilde{\tau}_{1} \operatorname{Re} \mathcal{A}\right)^{2}+|\tilde{\tau}|^{2}(\operatorname{Im} \mathcal{A})^{2}}}{\mathcal{B}-\tilde{\tau}_{1} \overline{\mathcal{A}}} .
$$

Substituting into (5.44), we find that (5.43) is given, in the semi-classical approximation, by the expression

$$
J t_{s}^{-\frac{\chi(\hat{\mathcal{X}})}{24}} e^{-S\left(t_{s}\right)}
$$

where the classical action is given by

$$
\begin{aligned}
S\left(t_{s}\right)= & \frac{2 \pi k \tau_{2}}{|\tilde{\tau}|^{2}} \sqrt{\left(\mathcal{B}-\tilde{\tau}_{1} \operatorname{Re} \mathcal{A}\right)^{2}+|\tilde{\tau}|^{2}(\operatorname{Im} \mathcal{A})^{2}} \\
& +2 \pi \mathrm{i} k\left[\frac{1}{2}\left(\sigma+\left(\zeta^{\Lambda}-2 n^{\Lambda}\right) \tilde{\zeta}_{\Lambda}^{\prime}\right)-\frac{\tau_{2}^{2}}{4} \operatorname{Re}\left(\bar{z}^{\Lambda} F_{\Lambda}^{\prime}\right)+|\tilde{\tau}|^{-2}\left(\tau_{2}^{2} \operatorname{Re} \mathcal{A}+\tilde{\tau}_{1} \mathcal{B}\right)\right]
\end{aligned}
$$


and $J$ is defined as

$$
J=\frac{p-k \xi^{0}\left(t_{s}\right)}{|p-k \tau|}\left|W_{k, p, \hat{\gamma}}\right|^{-1 / 2}=\frac{|p-k \tau|\left|W_{k, p, \hat{\gamma}}\right|^{1 / 2}}{\left(p-k \tau_{1}\right)\left|W_{k, p, \hat{\gamma}}\right|-\mathrm{i} k \tau_{2} \operatorname{Re} W_{k, p, \hat{\gamma}}} .
$$

It is straightforward to check that (5.49) (plus the omitted constant terms) reproduces the action $S_{k, p . \hat{\gamma}}$ in (4.41) obtained by S-duality transformation of the standard D-instanton action (4.36).

It is also instructive to compute (5.43) directly in the weak coupling limit. Assuming that the integral is controlled by a saddle point with $t_{s} \sim 1 / \tau_{2}$ as $\tau_{2} \rightarrow \infty$ (which is indeed the case of (5.47)), we can redefine $t=t^{\prime} / \tau_{2}$ and expand the exponent in the limit $\tau_{2} \rightarrow \infty$, keeping $t^{\prime}$ finite. Neglecting the logarithmic contribution, we obtain that the argument of the exponential in (5.43) reduces to

$$
\begin{aligned}
S \approx & \pi \mathrm{i} k\left(\sigma+\left(\zeta^{\Lambda}-2 n^{\Lambda}\right) \tilde{\zeta}_{\Lambda}^{\prime}\right)+4 \pi k e^{\phi}-2 \pi \mathrm{i} \frac{p^{0}}{k} \hat{q}_{a} z^{a} \\
& -\pi k\left(\mathrm{i} \tau_{\Lambda \Sigma}\left(\zeta^{\Lambda}-n^{\Lambda}\right)\left(\zeta^{\Sigma}-n^{\Sigma}\right)+2 \tau_{2} t\left(\zeta^{\Lambda}-n^{\Lambda}\right) \operatorname{Im} \tau_{\Lambda \Sigma} \bar{z}^{\Sigma}-\frac{1}{2} \tau_{2}^{2} t^{2} \bar{z}^{\Sigma} \operatorname{Im} \tau_{\Lambda \Sigma} \bar{z}^{\Sigma}\right) .
\end{aligned}
$$

The saddle point for the $t$ integral lies at

$$
t_{s}=\frac{2\left(\zeta^{\Lambda}-n^{\Lambda}\right) \operatorname{Im} \tau_{\Lambda \Sigma} \bar{z}^{\Sigma}}{\tau_{2} \bar{z}^{\Lambda} \operatorname{Im} \tau_{\Lambda \Sigma} \bar{z}^{\Sigma}}=4 e^{\mathcal{K}} \tau_{2}^{-1}\left(\zeta^{\Lambda}-n^{\Lambda}\right) \operatorname{Im} \mathcal{N}_{\Lambda \Sigma} z^{\Sigma}
$$

consistently with the weak coupling limit of (5.47), so that the semi-classical approximation to (5.43) becomes

$$
\tau_{2}^{-1} t_{s}^{-1-\frac{\chi(\hat{\mathcal{X}})}{24}}\left(\bar{z}^{\Lambda} \operatorname{Im} \tau_{\Lambda \Sigma} \bar{z}^{\Sigma}\right)^{-1 / 2} e^{-S\left(t_{s}\right)}
$$

with

$$
S\left(t_{s}\right)=4 \pi k e^{\phi}+\pi \mathrm{i} k\left(\sigma+\left(\zeta^{\Lambda}-2 n^{\Lambda}\right) \tilde{\zeta}_{\Lambda}-\overline{\mathcal{N}}_{\Lambda \Sigma}\left(\zeta^{\Lambda}-n^{\Lambda}\right)\left(\zeta^{\Sigma}-n^{\Sigma}\right)\right)-2 \pi \mathrm{i} \frac{p^{0}}{k} \hat{q}_{a} z^{a} .
$$

The classical action (5.54) indeed reproduces the Gaussian fivebrane action (4.43), after using identifications (4.44). While this agreement was guaranteed given the fact that (5.49) equals $S_{k, p . \hat{\gamma}}$ which reduces to (4.43) at weak coupling, this computation allows us to make contact with the analysis in [32], where an auxiliary variable $t$ was introduced as a way to turn the Gaussian partition function in the Weil polarization into an indefinite Gaussian partition function in the Griffiths polarization: this auxiliary variable $t$ is just the coordinate on the $\mathbb{C} P^{1}$ fiber over $\mathcal{M}$, after a simple rescaling.

Eq. (5.51) also indicates that the non-Gaussian theta series (5.37) is formally divergent, as it reduces to an indefinite Gaussian sum in the regime of weak coupling. However, it should be kept in mind that each term in (5.37) is integrated on a different contour in $\mathbb{C} P^{1}$. Provided one first carries out the contour integral in $\mathbb{C} P^{1}$ and then the sum over charges, the end result will involve a sum of exponentially suppressed instantonic corrections, though each of them might be multiplied by an exponentially growing summation measure as in the D-instanton sector [27]. 


\subsubsection{Non-Gaussian fivebrane partition function}

We define the non-Gaussian fivebrane partition function by summing the Penrose transform evaluated in 5.5.1 over charges,

$$
\mathcal{Z}_{\mathrm{NS} 5}^{(k, \mu)} \equiv e^{\phi} \sum_{p, p^{a}, \hat{q}_{a}, \hat{q}_{0}} \int_{\ell_{k, p, \hat{\gamma}}} \frac{\mathrm{d} t}{t} H_{k, p, \hat{\gamma}}(\xi(t), \tilde{\xi}(t), \tilde{\alpha}(t)) .
$$

The fact that the Gaussian action (5.54) resulting from the integral and the omitted constant prefactor, both depend on charges $\hat{q}_{\Lambda}$ linearly, allows us to carry out the sum over electric charges explicitly. We now evaluate this sum for $k=1$ in terms of the partition function of DT invariants. Setting $n^{\Lambda}=0$ by Heisenberg invariance, we have $p^{0}=1$, $\hat{q}_{a}=q_{a}-\frac{c_{2, a}}{24}$ and so only one term in (5.54) contributes. Reinstating the summation measure $\bar{\Omega}(\gamma)$ and the quadratic refinement with the characteristics fixed as above, the sum over electric charges leads formally to

$$
\sum_{q_{a}, q_{0}} \bar{\Omega}(\gamma) \mathbf{E}\left(\hat{q}_{a} z^{a}-\frac{1}{2} \hat{q}_{0}\right)=\left.\mathbf{E}\left(-\frac{c_{2 a} z^{a}}{24}\right) Z_{\mathrm{DT}}\left(z^{a}, \lambda\right)\right|_{\lambda \rightarrow 0},
$$

where $Z_{\mathrm{DT}}$ is the DT-partition function (2.83). Using the DT/GW relation (2.84), this may be further rewritten as

$$
\left.\lambda^{\frac{\chi(\hat{\mathcal{X}})}{24} \epsilon_{\mathrm{GW}}}\left[M\left(e^{-\lambda}\right)\right]^{\left(\epsilon_{\mathrm{DT}}-\frac{1}{2}\right) \chi(\hat{\mathcal{X}})} e^{F_{\mathrm{hol}}+\frac{(2 \pi \mathrm{i})^{3}}{\lambda^{2}}\left(N\left(z^{a}\right)-\frac{1}{2} A_{\Lambda \Sigma} z^{\Lambda} z^{\Sigma}\right)}\right|_{\lambda \rightarrow 0} .
$$

In the limit $\lambda \rightarrow 0$, only genus 0 and genus 1 contributions to $F_{\text {hol }}$ remain. For $\epsilon_{\mathrm{DT}}=0$ the power of the Mac-Mahon function cancels the degenerate Gromov-Witten contributions in $F_{\text {hol }}$, while the total remaining power of $\lambda$ cancels for $\epsilon_{\mathrm{GW}}=1$. Still, the non-degenerate genus zero Gromov-Witten contributions seem to make the limit $\lambda \rightarrow 0$ singular. It is plausible that these singular contributions may be canceled when $\alpha^{\prime}$ and $\mathrm{D}(-1)$-instanton corrections to the Darboux coordinates are included, and we shall ignore them in what follows. Thus, we conclude that the expression (5.57) can be replaced by $e^{f_{1}(z)}$ where $f_{1}(z)$ is the holomorphic part of the one-loop vacuum amplitude $F_{1}(2.50)$.

As a result, we find that the weak coupling approximation of the fivebrane partition function is given by

$$
\mathcal{Z}_{\mathrm{NS} 5}^{(1)} \sim \tau_{2} e^{f_{1}-\mathcal{K}}\left(\bar{z}^{\Lambda} \operatorname{Im} \tau_{\Lambda \Sigma} \bar{z}^{\Sigma}\right)^{-1 / 2} \sum_{n \in \Gamma_{m}+\theta} t_{s}^{-1-\frac{\chi(\hat{\mathcal{X}})}{24}} e^{-2 \pi \mathrm{in} n^{\Lambda} \phi_{\Lambda}-S^{\prime}\left(t_{s}\right)},
$$

where $S^{\prime}\left(t_{s}\right)$ is the action (5.54) without the last term. Extracting the factor $e^{-4 \pi e^{\phi}-\mathrm{i} \pi \sigma}$, as in (3.8), we obtain the Gaussian NS5-partition function

$$
\mathcal{Z}_{\mathrm{NS} 5-\mathrm{G}}^{(1)} \equiv \sum_{n \in \Gamma_{m}+\theta} \mathcal{F} \mathbf{E}\left(\frac{1}{2}\left(\zeta^{\Lambda}-n^{\Lambda}\right) \overline{\mathcal{N}}_{\Lambda \Sigma}\left(\zeta^{\Sigma}-n^{\Sigma}\right)+n^{\Lambda}\left(\tilde{\zeta}_{\Lambda}-\phi_{\Lambda}\right)-\frac{1}{2} \zeta^{\Lambda} \tilde{\zeta}_{\Lambda}\right),
$$

where the prefactor $\mathcal{F}$ is given by

$$
\mathcal{F}(n ; \mathcal{N}, z, \phi, \zeta)=\frac{\left(e^{-\mathcal{K}} \tau_{2}\right)^{2+\frac{\chi(\hat{\mathcal{X}})}{24}}}{\sqrt{\bar{z}^{\Lambda} \operatorname{Im} \tau_{\Lambda \Sigma} \bar{z}^{\Sigma}}}\left[\left(\zeta^{\Lambda}-n^{\Lambda}\right) \operatorname{Im} \mathcal{N}_{\Lambda \Sigma} z^{\Sigma}\right]^{-1-\frac{\chi(\hat{\mathcal{X}})}{24}} e^{f_{1}(z)} .
$$


Comparing the result (5.59) with the analysis in section 2.2 shows that the dependence on the $C$-field is given by a generalized Siegel theta series, with an insertion of a power of $t_{s}$ (given in eq. (5.52)) in the sum. ${ }^{39}$ Thus, the assumption made in section 2.2 that the normalization factor $\mathcal{F}$ did not depend on the flux $H$ was erroneous. In retrospect, such flux-dependent insertions into the fivebrane theta series were already present in the automorphic studies [52, 54].

The fact that the holomorphic part $f_{1}(z)$ of the B-model one loop amplitude $F_{1}(2.50)$ appears in the prefactor (5.60) implies that the one-loop determinant of the non-chiral fivebrane partition function in the flux sector $H$ is proportional to $e^{F_{1}}$, as anticipated in [32]. The latter is a product of analytic Ray-Singer torsions [45],

$$
e^{F_{1}}=\prod_{0 \leq p, q \leq 3}\left[\operatorname{det}^{\prime} \Delta_{\bar{\partial}}^{p, q}\right]^{\frac{1}{2} p q(-1)^{p+q}}=\frac{\left(\operatorname{det}^{\prime} \Delta_{\bar{\partial}}^{0,0}\right)^{9 / 2}\left(\operatorname{det}^{\prime} \Delta_{\bar{\partial}}^{1,1}\right)^{1 / 2}}{\left(\operatorname{det}^{\prime} \Delta_{\bar{\partial}}^{1,0}\right)^{3}}
$$

where $\Delta_{\bar{\partial}}^{p, q}$ is the Laplacian on $p$-forms valued in $\Lambda^{q} T \mathcal{X}$, and $\operatorname{det}^{\prime}$ is the determinant with zero-modes removed. In the second equality we used the standard identities $\operatorname{det}^{\prime} \Delta_{\bar{\partial}}^{p, q}=$ $\operatorname{det}^{\prime} \Delta_{\bar{\partial}}^{3-p, q}=\operatorname{det}^{\prime} \Delta_{\bar{\partial}}^{q, p}$ (see e.g. [105]). The fact that this normalization factor differs from the one computed in $[35,81]$ should come as no surprise, since these authors considered the partition function of the chiral two-form, whereas our result applies to the partition function of the $(2,0)$ supersymmetric field theory on the NS5-brane with an insertion of $(-1)^{2 J_{3}}\left(2 J_{3}\right)^{2}$, as required for computing instanton corrections to the two-derivative low energy effective action. It would be very interesting to perform the one-loop determinant computation explicitly.

To summarize, we have found that the saddle point approximation of the Penrose transform (5.43) produces a refinement of the chiral Gaussian partition function constructed in section 2.2. It is therefore natural to consider the original Penrose transform as an extension of the Gaussian fivebrane partition function (5.59) in the regime where the energy stored in the three-form flux is of the same order or larger than the energy in the fivebrane itself. Building upon the discussion at the end of section 2.3, we thus define the full non-linear partition function of $k$ chiral NS5-branes as the integrated matrix element

$$
\mathcal{Z}_{\mathrm{NS} 5}^{(k, \mu)}=e^{\phi} \int \frac{\mathrm{d} t}{t} e^{-\mathrm{i} \pi k\left(\tilde{\alpha}+\xi^{\Lambda} \tilde{\xi}_{\Lambda}\right)}\left\langle\Psi^{\Gamma_{m}, k, \mu}\left|e^{\mathrm{i}\left(\xi^{\Lambda} \tilde{T}_{\Lambda}-\tilde{\xi}_{\Lambda} T^{\Lambda}\right)}\right| H_{\mathrm{NS} 5}^{(k, \mu)}\right\rangle,
$$

\footnotetext{
${ }^{39} \mathrm{It}$ is interesting to note that insertions of powers of $t_{s}$ in the sum do not spoil the modular properties of the theta series, at least in the semi-classical approximation. This is obvious for transformations of type (2.39) and (2.40). For the inversion $\overline{\mathcal{N}} \mapsto-\overline{\mathcal{N}}^{-1}$, it suffices to evaluate the Fourier transform

$$
\int \mathrm{d} \zeta^{\Lambda}\left(z^{\Lambda} \operatorname{Im} \mathcal{N}_{\Lambda \Sigma} \zeta^{\Sigma}\right)^{-1-\frac{\chi(\hat{\mathcal{X}})}{24}} e^{-\mathrm{i} \pi \zeta^{\Lambda} \overline{\mathcal{N}}_{\Lambda \Sigma} \zeta^{\Sigma}+2 \pi \mathrm{i} k \zeta^{\Lambda} \tilde{\zeta}_{\Lambda}}
$$

in the saddle point approximation. At the extremum $\zeta=\overline{\mathcal{N}}^{-1} \tilde{\zeta}$, we have $z^{\Lambda} \operatorname{Im} \mathcal{N}_{\Lambda \Sigma} \zeta^{\Sigma}=$ $\frac{1}{2 \mathrm{i}} z^{\Lambda}\left[\mathcal{N}\left(\overline{\mathcal{N}}^{-1}-\mathcal{N}^{-1}\right)\right]_{\Lambda}{ }^{\Sigma} \tilde{\zeta}_{\Sigma}=-F_{\Lambda}\left[\operatorname{Im}\left(\mathcal{N}^{-1}\right)\right]^{\Lambda \Sigma} \tilde{\zeta}_{\Sigma}$, where we used the identity $\mathcal{N}_{\Lambda \Sigma} z^{\Sigma}=F_{\Lambda}$, so that the integral becomes

$$
(\operatorname{det} \overline{\mathcal{N}})^{-1 / 2}\left(-F_{\Lambda}\left[\operatorname{Im}\left(\mathcal{N}^{-1}\right)\right]^{\Lambda \Sigma} \tilde{\zeta}_{\Sigma}\right)^{-1-\frac{\chi(\hat{\mathcal{X}})}{24}} e^{\mathrm{i} \pi \tilde{\zeta}_{\Lambda}\left(\overline{\mathcal{N}}^{-1}\right)^{\Lambda \Sigma} \tilde{\zeta}_{\Sigma}}
$$

consistently with the transformations properties of the various quantities involved.
} 
where $\Psi^{\Gamma_{m}, k, \mu}$ is the same distribution as in (2.43), and $\left|H_{\mathrm{NS} 5}^{(k, \mu)}\right\rangle$ is the state whose wavefunction in the real polarization is given by (5.37). Note that we are here including the dependence on the NS-axion $\sigma$ in the definition of the non-Gaussian partition function. This is quite natural given the observation at the end of section 3.2.3 that $\sigma$ is in fact not well defined by itself.

Finally, we note that the functional space where the generators $\tilde{T}_{\Lambda}$ and $T^{\Lambda}$ operate is recognized as a space of (local) holomorphic sections on $\mathcal{Z}_{\mathcal{M}}$. For supergravity theories with a symmetric moduli space, this same space is the habitat of the quaternionic discrete series representations, whose relevance to the issue of instanton corrections to the HM moduli space has been advocated previously $[2,52,54]$.

\section{Discussion}

In this work, we have taken steps towards understanding NS5-brane instanton corrections to the hypermultiplet moduli space $\mathcal{M}$ in type IIA string theory compactified on a CalabiYau threefold $\mathcal{X}$, and other moduli spaces related to it by T-duality and mirror symmetry (see table 1 on page 26).

Our first main result, announced previously in [1], is the identification of the topology of $\mathcal{M}$ around the weak coupling limit: $\mathcal{M}$ is a foliated by hypersurfaces $\mathcal{C}(r), r \in \mathbb{R}^{+}$, each of which being a circle bundle $\left(\mathcal{L}_{\Theta} \otimes \mathcal{L}^{\frac{\chi(\mathcal{X})}{24}}\right)^{\circ}$ over the intermediate Jacobian $\mathcal{J}_{c}(\mathcal{X})$. Recall from $(2.9)$ that $\mathcal{J}_{c}(\mathcal{X})$ is the total space of the torus bundle over the complex structure moduli space $\mathcal{M}_{c}(\mathcal{X})$ with fiber $\mathcal{T}=H^{3}(\mathcal{X}, \mathbb{R}) / H^{3}(\mathcal{X}, \mathbb{Z})$. Physically, $r \sim 1 / g_{(4)}^{2}$ denotes the $4 \mathrm{D}$ string coupling, $\mathcal{T}$ parametrizes the harmonic $C$-field on $\mathcal{X}$, and the circle fiber $S_{\sigma}^{1}$ of $\mathcal{C}(r)$ parametrizes the Neveu-Schwarz axion. The curvature of $\mathcal{C}(r)$ (1.3) has two components: i) the Kähler class $\omega_{\mathcal{T}}$ reflects the fact that translations on $\mathcal{T}$ commute up to a translation along the circle fiber $S_{\sigma}^{1}$, ii) while $\frac{\chi(\mathcal{X})}{24} \omega_{\mathcal{S K}}$ reflects the fact that $\sigma$ shifts under phase rotations of the holomorphic three-form $\Omega_{3,0}$ on $\mathcal{X}$ as well as under monodromies in $\mathcal{M}_{c}(\mathcal{X})$. In the strict weak coupling limit, $g_{(4)}=0$, the metric is invariant under continuous translations on $\mathcal{T}$ and $S_{\sigma}^{1}$, but these continuous isometries are broken to discrete identifications (3.15) by D-instantons and NS5-brane instantons, respectively. In particular, the identifications (3.15) and (3.22) involve a choice of quadratic refinement $\sigma_{\Theta}$ of the intersection form on $H^{3}(\mathcal{X}, \mathbb{Z})$, together with a unitary character $e^{2 \pi i \kappa(M)}$ of the monodromy group which, to our knowledge, had not been noticed previously (the former did however appear in [53], which was developed concurrently to the present work). The character $e^{2 \pi \mathrm{i} \kappa(M)}$ is related to the multiplier system of the one-loop amplitude of the topological B-model on $\mathcal{X}$, and enters in the definition of the twisted line bundle $\mathcal{L}^{\chi(\mathcal{X}) / 24}$ where the NS-axion is valued.

The hypermultiplet moduli space in type IIB string theory compactified on the mirror CY threefold $\hat{\mathcal{X}}$ exhibits the same structure as in type IIA, as required by quantum mirror symmetry. The hypersurfaces $\mathcal{C}(r)$ are now circle bundles over the "symplectic Jacobian" $\mathcal{J}_{K}(\hat{\mathcal{X}})$ (4.19), which is the total space of a torus bundle over the Kähler moduli space $\mathcal{M}_{K}(\hat{\mathcal{X}})$ with fiber $H^{\text {even }}(\hat{\mathcal{X}}, \mathbb{R}) / K(\hat{\mathcal{X}})$. In particular, we have clarified the map from the K-theory lattice $K(\hat{\mathcal{X}})$ to the D-brane charge lattice $H^{\text {even }}(\hat{\mathcal{X}}, \mathbb{Z})$, by showing explicitly 
that it is given by a modification (4.20) of the standard generalized Mukai map. This modified Mukai map crucially involves the quadratic real matrix $A_{\Lambda \Sigma}$ appearing in the prepotential (4.4). Due to the fractional value of the (primed) $\mathrm{D}(-1)$-brane charge, we furthermore found it necessary to modify the action of S-duality given in $[14,18,20]$ on the D3-brane axion $c_{a}$, by a shift proportional to the multiplier system of the Dedekind eta function. Of course, the same considerations also apply for D6-D4-D2-D0 branes in type IIA string theory compactified on the CY threefold $\hat{\mathcal{X}}$.

Using S-duality, we inferred the qualitative form of $(p, k) 5$-brane corrections to the metric, and verified agreement with the type IIA Gaussian result in the weak coupling limit. It should be stressed that although the type IIB fivebrane is non-chiral, its partition function can still be written as a theta series of a similar form as in the type IIA picture. In particular, it must involve a quadratic refinement $\sigma_{\Theta}$, which can in principle be computed by S-duality from the quadratic refinement $\sigma_{\Theta_{\mathrm{D}}}$ governing D-instantons. It would be interesting to understand the origin of this apparent "chirality" on the type IIB NS5-brane worldvolume, perhaps along the lines of [106]. The independence of the physical hypermultiplet metric on the choice of quadratic refinement (as is known to be the case in $\mathcal{N}=2$ field theories [24]) further suggests that the NS5- and D-instanton characteristics $\Theta$ and $\Theta_{D}$ should be equal. On the other hand, our naive implementation of Heisenberg and S-duality indicates a different answer, eq. (A.7) and (A.9). It is an important open problem to resolve this discrepancy.

To implement NS5-brane instanton corrections consistently with supersymmetry, it is necessary to reformulate them in terms of deformations of the complex contact structure on the twistor space $\mathcal{Z}_{\mathcal{M}}$ over $\mathcal{M}$. At the perturbative level and over a fixed point in $\mathcal{M}_{c}(\mathcal{X})$, $\mathcal{Z}_{\mathcal{M}}$ can be obtained as the quotient of $H^{3}(\mathcal{X}, \mathbb{C}) \times \mathbb{C}$ by the discrete Heisenberg identifications (5.13). Corrections to the perturbative metric are then encoded in holomorphic sections of $H^{1}\left(\mathcal{Z}_{\mathcal{M}}, \mathcal{O}(2)\right)$. As alluded to above, by applying S-duality to the holomorphic sections describing D5-D3-D1-D(-1) instanton corrections in type IIB, we constructed a candidate section $H_{k, p, \hat{\gamma}}$ in eq. (5.30) encoding the contribution of a charge $k$ NS5-brane instanton. The resulting deformation of the contact structure is formally invariant under Heisenberg shifts and under monodromies around the large volume point, up to phases $\nu(\eta)$ and $\nu^{\prime}(\epsilon)$ computed in appendix A. The most conservative explanation of this clash is probably that our assumption of the invariance of the transition functions is too restrictive, and one must allow for local compensating contact transformations, as already observed in [20]. It is also conceivable that one (or more) of the symmetries must give in. For instance, one might expect that S-duality is broken to a finite index subgroup, as is sometimes the case of electric-magnetic duality in $\mathcal{N}=2$ field theories.

In spite of the problems mentioned above, our results nonetheless indicate that our approach is reasonable. In particular, we find that the single fivebrane wave-function $H_{\mathrm{NS5}}^{(1,0)}(5.37)$ is proportional to the wave-function $\Psi_{\mathbb{R}}^{\text {top }}$ of the topological B-model in the real polarization, up to certain factors which deserve further study (see (5.42)). Moreover, its Penrose transform reproduces a variant of the Gaussian flux partition function with a normalization factor proportional to the one-loop B-model amplitude, consistent with the topology of the one-loop corrected HM moduli space. It would be interesting to verify this 
result by a direct computation of the twisted partition function of the $(2,0)$ field theory on the fivebrane worldvolume, and elucidate the origin of the flux-dependent insertion in the Gaussian sum.

While we feel that the above results constitute significant progress towards understanding fivebrane instanton corrections, they are still far from providing the exact metric on the HM moduli space. In particular, our prescription for "summing" over images under $\mathrm{SL}(2, \mathbb{Z})$ formally leads to a dense set of mutually intersecting "BPS rays" on the twistor fiber at fixed values of the moduli, whose mathematical status is unclear. While the twistorial construction of NS5-brane instantons presented in this paper is adapted to type IIB S-duality, it is natural to wonder if there exists an alternative construction, more suitable for type IIA, which would make symplectic invariance manifest, and hopefully remove the dense set of contours mentioned above, as in the D1-D(-1) sector considered in [20].

It is also important to understand how our prescription is consistent with wall-crossing. Indeed, one of the hints in uncovering the structure of the D-instanton corrections [18-20] was the Kontsevich-Soibelman wall-crossing formula [25], which involves a product of symplectomorphisms: as shown in [24], the KS formula finds a natural interpretation in the twistorial description of the hyperkähler moduli space of $\mathcal{N}=2$ gauge theories on $\mathbb{R}^{3} \times S^{1}$. In the presence of NS5-brane instantons we require a similar formula now involving a product of contact transformations, perhaps arising from a suitable limit of the motivic wall-crossing formula of [25]. We hope to return to these issues in future work.

\section{Acknowledgments}

We are especially grateful to G. Moore for numerous clarifying discussions. We also thank M. Douglas, J. Evslin, D. Jatkar, A. Kleinschmidt, J. Manschot, R. Minasian, S. Monnier, A. Neitzke, B. Nilsson, C. Petersson, Y. Soibelman, S. Vandoren, P. Vanhove, A. Wijns and D. Zagier for valuable discussions and correspondence. S.A. is grateful to Perimeter Institute for Theoretical Physics for the kind hospitality and the financial support. D.P. is grateful to LPTHE for hospitality where part of this work was carried out. D.P. and B.P. acknowledge the hospitality of the Chalmers University of Technology, Göteborg University and the Marine Biological Laboratory in Tjärnö during the final stage of this project.

\section{A Action of Heisenberg shifts and monodromies on $\boldsymbol{H}_{k, p, \hat{\gamma}}$}

In this appendix we derive how the transition functions (5.30) encoding NS5-brane corrections transform under the Heisenberg action and monodromies around the large volume point in $\mathcal{M}_{K}(\hat{\mathcal{X}})$. 


\section{A.1 Heisenberg symmetry}

Let us start from the transition function (5.30) obtained by S-duality on the D-instanton series. It can be presented in the following form

$$
\begin{aligned}
H_{k, p, \hat{\gamma}}= & \frac{1}{4 \pi^{2}} \bar{\Omega}(\gamma) \mathbf{E}\left(-\frac{k}{2}\left(\tilde{\alpha}+\xi^{\Lambda} \tilde{\xi}_{\Lambda}\right)+k n^{\Lambda}\left(\tilde{\xi}_{\Lambda}-\phi_{\Lambda}\right)-k \frac{N\left(\xi^{a}-n^{a}\right)}{\xi^{0}-n^{0}}\right. \\
& +\frac{k}{2} A_{\Lambda \Sigma}\left(\xi^{\Lambda}-n^{\Lambda}\right)\left(\xi^{\Sigma}-n^{\Sigma}\right)+\frac{p^{0}\left(k \hat{q}_{a}\left(\xi^{a}-n^{a}\right)+p^{0} \hat{q}_{0}\right)}{k^{2}\left(\xi^{0}-n^{0}\right)} \\
& \left.+a \frac{p^{0} q_{0}^{\prime}}{k}-c_{2, a} p^{a} \varepsilon(\delta)-\frac{k}{2} A_{\Lambda \Sigma} n^{\Lambda} n^{\Sigma}-\frac{1}{2} q_{\Lambda} p^{\Lambda}+q_{\Lambda} \theta_{\mathrm{D}}^{\Lambda}-p^{\Lambda} \phi_{\mathrm{D}, \Lambda}+k n^{\Lambda} \phi_{\Lambda}\right),
\end{aligned}
$$

where we distinguished between characteristics appearing in the D-instanton series, which we denoted by $\left(\theta_{\mathrm{D}}^{\Lambda}, \phi_{\mathrm{D}, \Lambda}\right)$, and the characteristics appearing in the Heisenberg symmetry transformation (5.13), $\left(\theta^{\Lambda}, \phi_{\Lambda}\right)$, which are relevant for NS5-branes. Note that the two terms proportional to the matrix $A_{\Lambda \Sigma}$ come from the difference between $\tilde{\xi}_{\Lambda}^{\prime}$ appearing in $S_{\alpha}$, eq. (5.31), and $\tilde{\xi}_{\Lambda}$ appearing in the Heisenberg action (5.13).

It is easy to see that invariance of $H_{k, p, \hat{\gamma}}$ under Heisenberg shifts (5.13) with $\eta^{\Lambda}=0$ is ensured provided the characteristics $\theta^{\Lambda}$ are set to zero. Thus, it remains to consider Heisenberg shifts with $\tilde{\eta}_{\Lambda}=\kappa=0$. It is clear that the shift $\xi^{\Lambda} \mapsto \xi^{\Lambda}+\eta^{\Lambda}$ should be compensated by a similar shift of the charges $n^{\Lambda}$. The shift $n^{0} \mapsto n^{0}+\eta^{0}$ corresponds to a S-duality transformation changing $p \mapsto p+k \eta^{0}$, whereas the shift $n^{a} \mapsto n^{a}+\eta^{a}$ amounts to a spectral flow transformation (4.24) on the magnetic charges $p^{a}$, with flow parameter $\epsilon^{a}=k \eta^{a} / p^{0}$. Applying the rest of the spectral flow transformation (4.24) to the charges $q_{a}, q_{0}$, and using the invariance of $\hat{q}_{a}, \hat{q}_{0}$, one observes that the first two lines in (A.1) are explicitly invariant under this combined action provided $\bar{\Omega}(\gamma)$ is invariant under the spectral flow, $\bar{\Omega}(\gamma[\epsilon])=\bar{\Omega}(\gamma)$. As a result, under this crucial assumption, we need to evaluate the transformation of only the constant terms in the last line and the full variation of $H_{k, p, \hat{\gamma}}$ takes the form

$$
H_{k, p+k \eta^{0}, \hat{\gamma}[\epsilon]}\left(\xi^{\Lambda}+\eta^{\Lambda}, \tilde{\xi}_{\Lambda}, \tilde{\alpha}+\eta^{\Lambda}\left(\tilde{\xi}_{\Lambda}-2 \phi_{\Lambda}\right)\right)=\nu(\eta) H_{k, p, \hat{\gamma}}\left(\xi^{\Lambda}, \tilde{\xi}_{\Lambda}, \tilde{\alpha}\right)
$$

where $\nu(\eta)$ is the phase factor to be found.

We split the phase $\nu(\eta)$ into three contributions. The first one is given by the variation of the quadratic refinement

$$
\begin{aligned}
\nu_{1}(\epsilon) \equiv \frac{\sigma_{\mathrm{D}}(\gamma[\epsilon])}{\sigma_{\mathrm{D}}(\gamma)}= & \mathbf{E}\left(\frac{1}{2} \kappa_{a b c} p^{a} p^{b} \epsilon^{c}-\frac{3}{2}\left(p^{0}\right)^{2} L_{0}(\epsilon)+\frac{1}{2}\left(p^{0}\right)^{2} L_{a}(\epsilon) \epsilon^{a}-p^{0} \epsilon^{a} \phi_{\mathrm{D}, a}\right. \\
& \left.-\theta_{\mathrm{D}}^{a}\left(\kappa_{a b c} p^{b} \epsilon^{c}+p^{0} L_{a}(\epsilon)\right)-\theta_{\mathrm{D}}^{0}\left(\epsilon^{a} q_{a}+p^{a} L_{a}(\epsilon)-L_{0}(\epsilon)-(p \epsilon \epsilon)\right)\right),
\end{aligned}
$$

where $L_{0}, L_{a}$ are the integer valued functions defined in (4.14). Second, the variation of 
the first two terms in the last line of (A.1) is given by

$$
\begin{aligned}
\nu_{2}\left(\epsilon, \eta^{0}\right) \equiv & \mathbf{E}\left(-c_{2, a}\left(p^{0} \epsilon^{a} \varepsilon\left(\delta\left[\eta^{0}\right]\right)+p^{a}\left(\epsilon\left(\delta\left[\eta^{0}\right]\right)-\epsilon(\delta)\right)+a \frac{p^{0}\left(q_{0}^{\prime}[\epsilon]-q_{0}^{\prime}\right)}{k}\right)\right. \\
= & \mathbf{E}\left(p^{0} c_{2, a} \epsilon^{a}\left(\frac{p}{24 k}+\frac{1}{2} s\left(\frac{p}{p^{0}},-\frac{k}{p^{0}}\right)+\frac{1}{8}\right)\right. \\
& \left.\quad+\frac{a p^{0}}{k}\left(p^{\Lambda} L_{\Lambda}(\epsilon)-q_{a} \epsilon^{a}+2 A_{a b} \epsilon^{a} p^{b}\right)+\left(p^{a}+p^{0} \epsilon^{a}\right) \frac{c_{2, a}}{24} \eta^{0}\right),
\end{aligned}
$$

where $q_{0}^{\prime}[\epsilon] \equiv q_{0}[\epsilon]-A_{0 \Lambda} p^{\Lambda}[\epsilon], \delta\left[\eta^{0}\right]$ is the $\mathrm{SL}(2, \mathbb{Z})$ matrix (5.28) with $d$ replaced by $d-c \eta^{0}$, and we used the fact that the Dedekind sum (4.35) satisfies $s\left(d-\eta^{0} c, c\right)=s(d, c)$. Finally, the third term in the same line generates the following contribution

$$
\begin{aligned}
\nu_{3}\left(\epsilon, \eta^{0}\right) & \equiv \mathbf{E}\left(-\frac{k}{2}\left(\eta^{\Lambda}+2 n^{\Lambda}\right) A_{\Lambda \Sigma} \eta^{\Sigma}\right) \\
& =\mathbf{E}\left(-\frac{p^{0}}{2 k} A_{a b}\left(p^{0} \epsilon^{a}+2 p^{a}\right) \epsilon^{b}-\frac{c_{2, a}}{24}\left(\frac{p p^{0}}{k} \epsilon^{a}+p^{a} \eta^{0}+p^{0} \epsilon^{a} \eta^{0}\right)-\frac{k}{2} A_{00}\left(\eta^{0}\right)^{2}\right) .
\end{aligned}
$$

As a result, the total variation of the transition function (A.1) is described by the following phase factor

$$
\begin{aligned}
\nu(\eta) \equiv & \mathbf{E}\left(k \eta^{\Lambda} \phi_{\Lambda}\right) \nu_{1}(\epsilon) \nu_{2}\left(\epsilon, \eta^{0}\right) \nu_{3}\left(\epsilon, \eta^{0}\right) \\
= & \mathbf{E}\left(k \eta^{0} \phi_{0}-\frac{k}{2} A_{00}\left(\eta^{0}\right)^{2}\right) \mathbf{E}\left(k \eta^{a}\left(\phi_{a}-\phi_{\mathrm{D}, a}\right)+\frac{k}{2 p^{0}}(p p \eta)+\frac{a p^{0}}{k} p^{\Lambda} L_{\Lambda}(\epsilon)\right. \\
& +\frac{k}{2} c_{2, a} \eta^{a}\left(s\left(\frac{p}{p^{0}},-\frac{k}{p^{0}}\right)+\frac{1}{4}\left(1-p^{0}\right)\right)-\frac{k(k+1)}{2} A_{a b} \eta^{a} \eta^{b}-\eta^{a} A_{a b} p^{b} \\
& \left.-\theta_{\mathrm{D}}^{a}\left(\kappa_{a b c} p^{b} \epsilon^{c}+p^{0} L_{a}(\epsilon)\right)-\theta_{\mathrm{D}}^{0}\left(\epsilon^{a} q_{a}+p^{a} L_{a}(\epsilon)-L_{0}(\epsilon)-(p \epsilon \epsilon)\right)\right) .
\end{aligned}
$$

One may try to cancel this phase by a suitable choice of characteristics. e.g. the first factor, which carries the dependence on $\eta^{0}$ and the only $q$-dependent term can be canceled by choosing, in a ad hoc fashion,

$$
\theta_{\mathrm{D}}^{0}=0, \quad \phi_{0}=\frac{1}{2} A_{00} .
$$

Note that vanishing of $\theta^{\Lambda}$ is required by the Heisenberg invariance and equivalently results from casting (5.34) into (5.35). For $k=1$, the phase simplifies in this case drastically into

$$
\nu_{k=1}(\eta)=\mathbf{E}\left(\eta^{a}\left(\phi_{a}-\phi_{\mathrm{D}, a}\right)-A_{a b} \eta^{a} \eta^{b}-\theta_{\mathrm{D}}^{a}\left(\kappa_{a b c} p^{b} \eta^{c}+p^{0} L_{a}(\eta)\right)\right),
$$

where we took into account that $p^{0}=1, s(p,-1)=0$. It may therefore be canceled completely by further choosing

$$
\theta_{\mathrm{D}}^{a}=0, \quad \phi_{a}-\phi_{\mathrm{D}, a}=A_{a a} .
$$

Thus, provided one takes $\theta^{\Lambda}=\theta_{\mathrm{D}}^{\Lambda}=0$ and (A.7) together with (A.9), for $k=1$, the Heisenberg symmetry can be kept unbroken. Unfortunately, the choices (A.7), (A.9) are inconsistent with the transformation properties of the characteristics under monodromies, as we now discuss. 


\section{A.2 Monodromy transformations}

Next we discuss the transformation of the transition functions (5.30) under monodromies $M$ around the large volume point in $\mathcal{M}_{K}(\hat{\mathcal{X}})$ which acts holomorphically on the twistor space by (5.12). It is clear that to compensate this transformation, one should supplement it by a spectral flow transformation of charges (4.24) where the transformation parameter is taken to be $\epsilon^{\prime a}=\left(p / p^{0}\right) \epsilon^{a}$. Under this simultaneous variation, the quantity $S_{\alpha}$ defined in (5.31) simply varies by a constant

$$
S_{\alpha} \mapsto S_{\alpha}+\kappa_{a b c} \epsilon^{a}\left[n^{b} n^{c}+n^{0} n^{b} \epsilon^{c}+\frac{1}{3}\left(n^{0}\right)^{2} \epsilon^{b} \epsilon^{c}\right] .
$$

In addition one should take into account that the monodromy induces a transformation of characteristics given in (2.24). It is explicit form can be obtained using the matrix $\rho(M)$ given in (4.25). Then taking into account that $\theta^{\Lambda}=0$, the characteristics undergo the following transformation

$$
\phi_{0} \mapsto \phi_{0}-\epsilon^{a} \phi_{a}-\frac{1}{2}\left(L_{0}(\epsilon)-\epsilon^{a} L_{a}(\epsilon)-(\epsilon \epsilon \epsilon)\right), \quad \phi_{a} \mapsto \phi_{a}+\frac{1}{2} \kappa_{a a c} \epsilon^{c},
$$

where we took into account that $\frac{1}{2}\left(\kappa_{a a c}-\epsilon^{b} \kappa_{a b c}\right) \epsilon^{a} \epsilon^{c} \in \mathbb{Z}$ and can be neglected.

As a result, assuming again that the invariants $\bar{\Omega}(\gamma)$ are not affected by the spectral flow, we can write the total variation as in (5.33) with the phase factor $\nu^{\prime}(\epsilon)$ given by

$$
\begin{aligned}
\nu^{\prime}(\epsilon)=\mathbf{E}( & -k \kappa_{a} \epsilon^{a}-\frac{k}{2} \delta_{\epsilon} S_{\alpha}+\frac{p^{0}}{k} \hat{q}_{a} \epsilon^{a}+p^{0} \epsilon^{a} \phi_{\mathrm{D}, a}+\frac{p^{0}}{2}\left(L_{0}(\epsilon)-\epsilon^{a} L_{a}(\epsilon)-(\epsilon \epsilon \epsilon)\right) \\
- & \left.\frac{1}{2}\left((p p \epsilon)+\frac{p}{p^{0}}(\epsilon \epsilon \epsilon)\right)\right) \nu_{1}\left(p \epsilon^{a} / p^{0}\right) \nu_{2}\left(p \epsilon^{a} / p^{0}, 0\right) .
\end{aligned}
$$

Unfortunately, it does not seem to be possible to dispose of this phase factor either. For $k=p^{0}=1$ it can be simplified to

$$
\nu_{k=1}^{\prime}(\epsilon)=\mathbf{E}\left(\frac{c_{2, a} \epsilon^{a}}{24}\left(3 p^{2}+2\right)-\frac{(p-1)^{2}}{2} A_{a b} \epsilon^{a} \epsilon^{b}-\left(\kappa_{a}+(p-1) \phi_{\mathrm{D}, a}\right) \epsilon^{a}\right)
$$

and does not vanish even in this particular situation.

Moreover, the transformation of the characteristics (A.11) seems to be inconsistent with the identifications (A.7) and (A.9). This shows that although the Heisenberg invariance can be achieved for $k=1$, there is a tension between the conditions ensuring this invariance and the monodromy transformations. In particular, this is why the considerations of the previous subsection do not necessarily imply that the D-instanton and fivebrane characteristics are not equal. 


\begin{tabular}{|c|c|}
\hline$\Psi_{\mathrm{BCOV}}$ & $\mathcal{L}^{1-\frac{\chi(\mathcal{X})}{24}}$ \\
$e^{f_{1}}$ & $\mathcal{L}^{1-\frac{\chi(\mathcal{X})}{24}+\frac{b_{3}}{4} \otimes K_{c}^{\frac{1}{2}}}$ \\
$e^{\mathcal{K}}$ & $\mathcal{L} \otimes \overline{\mathcal{L}}$ \\
$\Psi_{\mathrm{G}}$ & $\mathcal{L}^{\frac{b_{3}}{4}} \otimes K_{c}^{\frac{1}{2}}$ \\
$\Psi_{\mathrm{W}}$ & $\mathcal{L}^{\frac{b_{3}}{4}-1} \otimes K_{c}^{\frac{1}{2}}$ \\
$J_{\mathrm{G}}$ & $\mathcal{L}^{-b_{3} / 2} \otimes K_{c}^{-1} \otimes \operatorname{det}_{\mathrm{G}}^{-1}$ \\
$J_{\mathrm{W}}$ & $\mathcal{L}^{1-\frac{b_{3}}{2}} \otimes \overline{\mathcal{L}}^{-1} \otimes K_{c}^{-1} \otimes \overline{\operatorname{det}}_{\mathrm{W}}$ \\
$\operatorname{det}(-\operatorname{Im} \mathcal{N})$ & $\operatorname{det}_{\mathrm{W}}^{-1} \otimes \overline{\operatorname{det}_{\mathrm{W}}}-1$ \\
$\operatorname{det}(\operatorname{Im} \tau)$ & $\operatorname{det}_{\mathrm{G}}^{-1} \otimes \overline{\operatorname{det}_{\mathrm{G}}}-1$ \\
$X^{\Lambda} \operatorname{Im} \tau_{\Lambda \Sigma} X^{\Sigma}$ & $\mathcal{L}^{2} \otimes \operatorname{det}_{\mathrm{W}} \otimes \overline{\operatorname{det}_{\mathrm{G}}}-1$ \\
$X^{\Lambda} \operatorname{Im} \mathcal{N}_{\Lambda \Sigma} X^{\Sigma}$ & $\mathcal{L}^{2} \otimes \operatorname{det}_{\mathrm{G}} \otimes \overline{\operatorname{det}_{\mathrm{W}}}-1$ \\
$e^{-\frac{\chi(\mathcal{X})}{48} \mathcal{K}+\mathrm{i} \pi \sigma}$ & $\mathcal{L}_{\Theta} \otimes \mathcal{L}^{\chi / 24}$ \\
\hline
\end{tabular}

Table 2. Transformation properties of various quantities under $\mathcal{L} \otimes K_{c} \otimes \operatorname{det}_{\mathrm{G}} \otimes \operatorname{det}_{\mathrm{W}}$.

\section{B List of notations}

Here we present a list of the most important notations used throughout the paper (roughly in order of appearance):

$\mathbf{E}(z) \quad \exp [2 \pi \mathrm{i} z]$

$(\mathcal{X}, \hat{\mathcal{X}}) \quad$ mirror pair of Calabi-Yau threefolds

$\mathcal{M} \mathcal{Q}_{c}(\mathcal{X})($ IIA $)$ or $\mathcal{Q}_{K}(\hat{\mathcal{X}})($ IIB), quaternion-Kähler manifold

$\mathcal{Q}_{c}(\mathcal{X})$ hypermultiplet moduli space in type IIA on $\mathcal{X}$

$\mathcal{Q}_{K}(\mathcal{X})$ hypermultiplet moduli space in type IIB on $\hat{\mathcal{X}}$

$\mathcal{S K} \quad \mathcal{M}_{c}(\mathcal{X})($ IIA $)$ or $\mathcal{M}_{K}(\hat{\mathcal{X}})($ IIB), special Kähler manifold

$\mathcal{M}_{c}(\mathcal{X}) \quad$ complex structure moduli space of $\mathcal{X}$

$\mathcal{M}_{K}(\hat{\mathcal{X}}) \quad$ (complexified) Kähler moduli space of $\hat{\mathcal{X}}$

$\mathcal{K} \quad$ Kähler potential for the special Kähler metric on $\mathcal{S K}$

$\omega_{\mathcal{S K}} \quad$ Kähler form on $\mathcal{S K}$

$K_{c} \quad$ canonical bundle over $\mathcal{S K}$

$\tau_{\Lambda \Sigma} \quad$ period matrix in Griffiths complex structure $\left(\Lambda, \Sigma=0,1, \ldots, h_{2,1}\right)$

$\mathcal{N}_{\Lambda \Sigma} \quad$ period matrix in Weil complex structure (eq. (2.12))

$\mathcal{T} \quad \operatorname{torus} H^{3}(\mathcal{X}, \mathbb{R}) / H^{3}(\mathcal{X}, \mathbb{Z})(\mathrm{IIA}), H^{\text {even }}(\hat{\mathcal{X}}, \mathbb{R}) / K(\mathcal{X})(\mathrm{IIB})$

$\Gamma \quad$ charge lattice, $H^{3}(\mathcal{X}, \mathbb{Z})($ IIA) or $K(\mathcal{X})$ (IIB), or its Poincaré dual

$\left\langle\gamma, \gamma^{\prime}\right\rangle \quad q_{\Lambda} p^{\prime \Lambda}-p^{\Lambda} q_{\Lambda}^{\prime}$, integer symplectic pairing on $\Gamma$

$\Gamma_{e}, \Gamma_{m} \quad$ electric and magnetic charge sublattices of $\Gamma=\Gamma_{e} \oplus \Gamma_{m}$, isotropic 


\begin{tabular}{|c|c|}
\hline$\omega_{\mathcal{T}}$ & Kähler form on $\mathcal{T}$, (eq. (2.8)) \\
\hline $\mathcal{J}_{c}(\mathcal{X})$ & intermediate Jacobian: total space of $\mathcal{T} \rightarrow \mathcal{J}_{c}(\mathcal{X}) \rightarrow \mathcal{M}_{c}(\mathcal{X})$ \\
\hline $\mathcal{J}_{K}(\hat{\mathcal{X}})$ & symplectic Jacobian: total space of $\mathcal{T} \rightarrow \mathcal{J}_{K}(\hat{\mathcal{X}}) \rightarrow \mathcal{M}_{K}(\hat{\mathcal{X}})$ \\
\hline$\Omega_{3,0}$ & holomorphic 3 -form on $\mathcal{X}$ \\
\hline $\mathcal{L}$ & Hodge line bundle $\mathcal{L} \rightarrow \mathcal{M}_{c}(\mathcal{X})$ where $\Omega_{3,0}$ is valued \\
\hline $\mathcal{L}_{\Theta}$ & "theta line bundle" $\mathcal{L}_{\Theta} \rightarrow \mathcal{T}$, defined by periodicity (eq. (2.36)) \\
\hline $\mathcal{C}_{\Theta}$ & unit circle bundle $\mathcal{L}_{\Theta}^{\circ}$ inside $\mathcal{L}_{\Theta}$ \\
\hline $\mathcal{Z}_{\mathrm{G}}^{(k)}=\mathcal{Z}_{\Theta, \mu}^{(k)}$ & $\begin{array}{l}\text { Gaussian partition function of a chiral 3-form obtained by } \\
\text { holomorphic factorization }\end{array}$ \\
\hline$\underset{\mathcal{Z}_{\mathrm{NS} 5}^{(k)}}{\mathcal{Z}_{k}}$ & non-linear NS5-partition function governing instanton corrections \\
\hline $\mathcal{Z}_{\mathrm{NS} 5-\mathrm{G}}^{(k)}$ & weak-coupling limit of $\mathcal{Z}_{\mathrm{NS} 5}^{(k)} /\left[e^{-4 \pi|k| e^{\phi}-\pi \mathrm{i} k \sigma}\right]$ \\
\hline $\mathcal{F}$ & metric-dependent normalization of $\mathcal{Z}_{\Theta, \mu}^{(k)}$ \\
\hline $\mathcal{C}_{\mathrm{NS} 5}$ & circle bundle $\mathcal{C}_{\mathrm{NS} 5} \rightarrow \mathcal{J}_{c}(\mathcal{X})$ of which $\mathcal{Z}_{\mathrm{NS} 5}^{(1)}$ is a section \\
\hline$\Theta=\left(\theta^{\Lambda}, \phi_{\Lambda}\right)$ & characteristics of the theta function $\mathcal{Z}_{\Theta}^{(k)}$ \\
\hline$\sigma_{\Theta}$ & quadratic refinement of the intersection form on $H^{3}(\mathcal{X}, \mathbb{Z})$ modulo 2 \\
\hline$\left(\mathcal{A}^{\Lambda}, \mathcal{B}_{\Lambda}\right)$ & symplectic basis of $H_{3}(\mathcal{X}, \mathbb{Z})\left(\Lambda=0,1, \ldots, h_{2,1}\right)$ \\
\hline$\left(\alpha_{\Lambda}, \beta^{\Lambda}\right)$ & symplectic basis of $H^{3}(\mathcal{X}, \mathbb{Z})$ \\
\hline C & RR 3-form potential in $D=10$ type IIA string theory \\
\hline$B$ & 2 -form in $D=4$ spacetime \\
\hline $\mathcal{B}$ & "chiral" 2-form on the worldvolume $\mathcal{X}$ of the NS5-brane \\
\hline$H=d \mathcal{B}$ & imaginary self-dual field strength of $\mathcal{B}$ \\
\hline$C=\left(\zeta^{\Lambda}, \tilde{\zeta}_{\Lambda}\right)$ & periods of $C$ along $\left(\mathcal{A}^{\Lambda}, \mathcal{B}_{\Lambda}\right)$, "RR-axions" (eq. (2.6)) \\
\hline$\Omega=\left(X^{\Lambda}, F_{\Lambda}\right)$ & periods of $\Omega_{3,0}$ along $\mathcal{A}^{\Lambda}, \mathcal{B}_{\Lambda}$ (eq. (2.2)) \\
\hline$z^{a}=X^{a} / X^{0}$ & $\begin{array}{l}\text { projective coordinates on } \mathcal{M}_{c}(\mathcal{X})=\mathcal{M}_{K}(\hat{\mathcal{X}}) \\
\left(a=1, \ldots, h_{2,1}(\mathcal{X})=h_{1,1}(\hat{\mathcal{X}})\right)\end{array}$ \\
\hline$z^{\Lambda}$ & $\left(1, z^{a}\right)$ \\
\hline$F(X)$ & prepotential, $F_{\Lambda}=\partial_{X^{\Lambda}} F(X)$ \\
\hline$\sigma$ & dual of the 2-form $B$ ("NS-axion") \\
\hline$H=\left(n^{\Lambda}, m_{\Lambda}\right)$ & integral periods of $H$ along $\left(\mathcal{A}^{\Lambda}, \mathcal{B}_{\Lambda}\right)$ \\
\hline$\chi(\mathcal{X})$ & Euler number of $\mathcal{X}$ \\
\hline$r=e^{\phi}=g_{(4)}^{-2}$ & four-dimensional dilaton $\phi$ and string coupling $g_{(4)}$ \\
\hline$\tau_{2}=1 / g_{s}^{2}$ & ten-dimensional type IIB string coupling, related to $\phi$ via eq. (4.30) \\
\hline$\Psi_{\mathbb{R}}$ & wave function in the real polarization \\
\hline$F_{1}$ & one-loop topological string vacuum amplitude \\
\hline$f_{1}$ & holomorphic part of $F_{1}$ (eq. (2.50)) \\
\hline$\kappa(M)$ & logarithm of a unitary character of the monodromy group of $\mathcal{M}_{c}$ \\
\hline$\gamma$ & $\begin{array}{l}\text { charge vector (Mukai vector), valued in } H_{3}(\mathcal{X}, \mathbb{Z}), H_{\text {even }}(\hat{\mathcal{X}}, \mathbb{Z}) \\
\text { for IIA, IIB }\end{array}$ \\
\hline
\end{tabular}




\begin{tabular}{|c|c|}
\hline$Z_{\gamma}$ & central charge $e^{\mathcal{K} / 2}\left(q_{\Lambda} X^{\Lambda}-p^{\Lambda} F_{\Lambda}\right)$ (stability data) \\
\hline$W_{\gamma}$ & rescaled central charge, $W_{\gamma}=\frac{\tau_{2}}{2} e^{-\mathcal{K} / 2} Z_{\gamma}$ \\
\hline $\mathcal{C}(r)$ & circle bundle $\mathcal{C}(r) \rightarrow \mathcal{J}_{c}(\mathcal{X})$ (IIA), or $\mathcal{C}(r) \rightarrow \mathcal{J}_{K}(\hat{\mathcal{X}})\left(\right.$ IIB), with fiber $S_{\sigma}^{1}$ \\
\hline$N\left(X^{a}\right)$ & $\frac{1}{6} \kappa_{a b c} X^{a} X^{b} X^{c}$ \\
\hline$\omega_{\hat{\mathcal{X}}}, \omega^{a}, \omega_{a}, 1$ & symplectic basis of $H^{6}(\hat{\mathcal{X}}) \oplus H^{4}(\hat{\mathcal{X}}) \oplus H^{2}(\hat{\mathcal{X}}) \oplus H^{0}(\hat{\mathcal{X}})$ \\
\hline$\hat{\mathcal{X}}, \gamma_{a}, \gamma^{a},[\mathrm{pt}]$ & symplectic basis of $H_{6}(\hat{\mathcal{X}}) \oplus H_{4}(\hat{\mathcal{X}}) \oplus H_{2}(\hat{\mathcal{X}}) \oplus H_{0}(\hat{\mathcal{X}})$ \\
\hline$A_{\Lambda \Sigma}$ & real symmetric matrix defined up to integer shifts (eq. (4.4)) \\
\hline$c_{2, a}$ & $\int_{\hat{\mathcal{X}}} c_{2}(\hat{\mathcal{X}}) \omega_{a}$ \\
\hline$\gamma=\left(p^{\Lambda}, q_{\Lambda}\right)$ & $\begin{array}{l}\text { integer magnetic and electric D2-brane charges } \\
\text { (resp. D(-1)-D1-D3-D5 in IIB) }\end{array}$ \\
\hline$q_{\Lambda}^{\prime}$ & primed electric charges, $\mathbb{Q}$-valued (eq. (4.9)) \\
\hline$\zeta^{\prime \Lambda}$ & primed RR axions (eq. (4.18)) \\
\hline$\hat{q}_{\Lambda}$ & spectral flow-invariant combination of electric charges (eq. (4.26)) \\
\hline$\hat{\gamma}$ & reduced charge vector $\left(p^{a}, \hat{q}_{a}, \hat{q}_{0}\right)$ \\
\hline$Q_{a}, J$ & electric charge and momentum of 5D black hole (eq. (4.28)) \\
\hline$A^{\text {even }}$ & type IIB RR potential \\
\hline$\tau^{1}, c^{a}, c_{a}, c_{0}, \psi$ & RR and NS axions adapted to S-duality (eq. (4.29)) \\
\hline$k$ & integer NS5-brane charge \\
\hline $\mathcal{Z}$ & twistor space of $\mathcal{M}$, a fibration $\mathcal{Z} \rightarrow \mathcal{M}$ with fiber $\mathbb{C} P^{1}$ \\
\hline $\mathcal{U}_{i}$ & open covering of $\mathcal{Z}$ \\
\hline $\mathcal{X}^{[i]}$ & complex contact one-form (eq. (5.2)) \\
\hline$\Phi_{[i]}$ & contact potential (eq. (5.2)) \\
\hline $\mathcal{K}_{\mathcal{Z}}^{[i]}$ & Kähler potential on $\mathcal{Z}$ (eq. (5.3)) \\
\hline$\left(x^{\mu}, t\right)$ & generic coordinates on $\mathcal{M} \times \mathbb{C} P^{1}$ \\
\hline$\left(\xi_{[i]}^{\Lambda}, \tilde{\xi}_{\Lambda}^{[i]}, \alpha^{[i]}\right)$ & complex Darboux coordinates on $\mathcal{U}_{i} \subset \mathcal{Z}, \mathcal{X}^{[i]}=\mathrm{d} \alpha^{[i]}+\xi_{[i]}^{\Lambda} \mathrm{d} \tilde{\xi}_{\Lambda}^{[i]}$ \\
\hline$\tilde{\alpha}$ & $-2 \alpha-\xi^{\Lambda} \tilde{\xi}_{\Lambda}$, symplectic invariant Darboux coordinate \\
\hline$H^{[i j]}$ & holomorphic section of $H^{1}(\mathcal{Z}, \mathcal{O}(2))$, controlling deformations of $\mathcal{Z}$ \\
\hline$H_{\gamma}$ & $\begin{array}{l}\text { complex symplectomorphism encoding deformations of } \mathcal{Z} \text { due } \\
\text { to D-branes }\end{array}$ \\
\hline$\ell_{\gamma}$ & "BPS ray", defined as $\left\{t: Z_{\gamma}\left(z^{a}\right) / t \in \mathrm{i} \mathbb{R}^{-}\right\}$ \\
\hline$\Omega(\gamma)$ & generalized Donaldson-Thomas invariants (Z्Z-valued) \\
\hline $\bar{\Omega}(\gamma)$ & generalized Donaldson-Thomas invariants ( $\mathbb{Q}$-valued) (eq. (5.21)) \\
\hline$N_{\mathrm{DT}}$ & standard (rank 1) Donaldson-Thomas invariants (Z्Z-valued) \\
\hline$Z_{\mathrm{DT}}$ & Donaldson-Thomas partition function \\
\hline$Z_{\mathrm{GW}}$ & Gromov-Witten partition function \\
\hline$\delta$ & $\mathrm{SL}(2, \mathbb{Z})$-transformation (eq. (4.39)) \\
\hline$\varepsilon(\delta)$ & multiplier system of the Dedekind $\eta$-function (eq. (4.34)) \\
\hline$s(d, c)$ & Dedekind sum (eq. (4.35)) \\
\hline
\end{tabular}


$W_{k, p, \hat{\gamma}} \quad$ image of D-instanton central charge $W_{\gamma}$ under $\delta$

$\ell_{k, p, \hat{\gamma}} \quad$ image of the BPS ray $\ell_{\gamma}$ under $\delta$

$H_{k, p, \hat{\gamma}}$ image of D-instanton transition function $H_{\gamma}$ under $\delta$

$H_{\mathrm{NS} 5}^{(k)} \quad$ formal sum of $H_{k, p, \hat{\gamma}}$ over all $p, \hat{\gamma}$

Open Access. This article is distributed under the terms of the Creative Commons Attribution Noncommercial License which permits any noncommercial use, distribution, and reproduction in any medium, provided the original author(s) and source are credited.

\section{References}

[1] S. Alexandrov, D. Persson and B. Pioline, On the topology of the hypermultiplet moduli space in type-II/CY string vacua, Phys. Rev. D 83 (2011) 026001 [arXiv:1009.3026] [SPIRES].

[2] M. Günaydin, A. Neitzke, B. Pioline and A. Waldron, BPS black holes, quantum attractor flows and automorphic forms, Phys. Rev. D 73 (2006) 084019 [hep-th/0512296] [SPIRES].

[3] K. Becker, M. Becker and A. Strominger, Five-branes, membranes and nonperturbative string theory, Nucl. Phys. B 456 (1995) 130 [hep-th/9507158] [SPIRES].

[4] J. Bagger and E. Witten, Matter Couplings in $\mathcal{N}=2$ Supergravity, Nucl. Phys. B 222 (1983) 1 [SPIRES].

[5] C.G. Callan Jr., J.A. Harvey and A. Strominger, Worldbrane actions for string solitons, Nucl. Phys. B 367 (1991) 60 [SPIRES].

[6] S. Cecotti, S. Ferrara and L. Girardello, Geometry of Type II Superstrings and the Moduli of Superconformal Field Theories, Int. J. Mod. Phys. A 4 (1989) 2475 [SPIRES].

[7] S. Ferrara and S. Sabharwal, Quaternionic Manifolds for Type II Superstring Vacua of Calabi-Yau Spaces, Nucl. Phys. B 332 (1990) 317 [SPIRES].

[8] D.R. Morrison, Mirror symmetry and the type-II string, Nucl. Phys. Proc. Suppl. 46 (1996) 146 [hep-th/9512016] [SPIRES].

[9] M. Roček, C. Vafa and S. Vandoren, Quaternion-Kähler spaces, hyperKähler cones and the c-map, math/0603048. MATH/0603048;

[10] A. Neitzke, B. Pioline and S. Vandoren, Twistors and Black Holes, JHEP 04 (2007) 038 [hep-th/0701214] [SPIRES].

[11] S. Alexandrov, B. Pioline, F. Saueressig and S. Vandoren, Linear perturbations of quaternionic metrics. I. The HyperKähler case, Lett. Math. Phys. 87 (2009) 225 [arXiv: 0806 .4620] [SPIRES].

[12] S. Alexandrov, B. Pioline, F. Saueressig and S. Vandoren, Linear perturbations of quaternionic metrics. II. The quaternionic-Kähler case, Commun. Math. Phys. 296 (2010) 353 [arXiv:0810.1675] [SPIRES].

[13] I. Antoniadis, S. Ferrara, R. Minasian and K.S. Narain, $R^{4}$ couplings in $M$ - and type-II theories on Calabi-Yau spaces, Nucl. Phys. B 507 (1997) 571 [hep-th/9707013] [SPIRES].

[14] H. Günther, C. Herrmann and J. Louis, Quantum corrections in the hypermultiplet moduli space, Fortsch. Phys. 48 (2000) 119 [hep-th/9901137] [SPIRES].

[15] I. Antoniadis, R. Minasian, S. Theisen and P. Vanhove, String loop corrections to the universal hypermultiplet, Class. Quant. Grav. 20 (2003) 5079 [hep-th/0307268] [SPIRES]. 
[16] D. Robles-Llana, F. Saueressig and S. Vandoren, String loop corrected hypermultiplet moduli spaces, JHEP 03 (2006) 081 [hep-th/0602164] [SPIRES].

[17] S. Alexandrov, Quantum covariant c-map, JHEP 05 (2007) 094 [hep-th/0702203] [SPIRES].

[18] S. Alexandrov, B. Pioline, F. Saueressig and S. Vandoren, D-instantons and twistors, JHEP 03 (2009) 044 [arXiv:0812.4219] [SPIRES].

[19] S. Alexandrov, D-instantons and twistors: some exact results, J. Phys. A 42 (2009) 335402 [arXiv: 0902.2761] [SPIRES].

[20] S. Alexandrov and F. Saueressig, Quantum mirror symmetry and twistors, JHEP 09 (2009) 108 [arXiv:0906.3743] [SPIRES].

[21] D. Robles-Llana, M. Roček, F. Saueressig, U. Theis and S. Vandoren, Nonperturbative corrections to $4 D$ string theory effective actions from $\mathrm{SL}(2, Z)$ duality and supersymmetry, Phys. Rev. Lett. 98 (2007) 211602 [hep-th/0612027] [SPIRES].

[22] F. Saueressig and S. Vandoren, Conifold singularities, resumming instantons and non-perturbative mirror symmetry, JHEP 07 (2007) 018 [arXiv: 0704.2229] [SPIRES].

[23] D. Robles-Llana, F. Saueressig, U. Theis and S. Vandoren, Membrane instantons from mirror symmetry, Commun. Num. Theor. Phys. 1 (2007) 681 [arXiv:0707.0838] [SPIRES].

[24] D. Gaiotto, G.W. Moore and A. Neitzke, Four-dimensional wall-crossing via three-dimensional field theory, Commun. Math. Phys. 299 (2010) 163 [arXiv:0807.4723] [SPIRES].

[25] M. Kontsevich and Y. Soibelman, Stability structures, motivic Donaldson-Thomas invariants and cluster transformations, arXiv:0811.2435.

[26] D. Joyce, Generalized Donaldson-Thomas invariants, arXiv:0910.0105 [SPIRES].

[27] B. Pioline and S. Vandoren, Large D-instanton effects in string theory, JHEP 07 (2009) 008 [arXiv:0904.2303] [SPIRES].

[28] E. Witten, Five-brane effective action in M-theory, J. Geom. Phys. 22 (1997) 103 [hep-th/9610234] [SPIRES].

[29] L. Dolan and C.R. Nappi, A modular invariant partition function for the fivebrane, Nucl. Phys. B 530 (1998) 683 [hep-th/9806016] [SPIRES].

[30] M. Henningson, B.E.W. Nilsson and P. Salomonson, Holomorphic factorization of correlation functions in (4k+2)-dimensional (2k)-form gauge theory, JHEP 09 (1999) 008 [hep-th/9908107] [SPIRES].

[31] M. Henningson, The quantum Hilbert space of a chiral two-form in $D=5+1$ dimensions, JHEP 03 (2002) 021 [hep-th/0111150] [SPIRES].

[32] R. Dijkgraaf, E.P. Verlinde and M. Vonk, On the partition sum of the NS five-brane, hep-th/0205281 [SPIRES].

[33] E. Diaconescu, G.W. Moore and D.S. Freed, The M-theory 3-form and E 8 gauge theory, hep-th/0312069 [SPIRES].

[34] G.W. Moore, Anomalies, Gauss laws and page charges in M-theory, Comptes Rendus Physique 6 (2005) 251 [hep-th/0409158] [SPIRES]. 
[35] D. Belov and G.W. Moore, Holographic action for the self-dual field, hep-th/0605038 [SPIRES].

[36] D.M. Belov and G.W. Moore, Type II actions from 11-dimensional Chern-Simons theories, hep-th/0611020 [SPIRES].

[37] I.A. Bandos et al., Covariant action for the super-five-brane of M-theory, Phys. Rev. Lett. 78 (1997) 4332 [hep-th/9701149] [SPIRES].

[38] M. Aganagic, J. Park, C. Popescu and J.H. Schwarz, World-volume action of the M-theory five-brane, Nucl. Phys. B 496 (1997) 191 [hep-th/9701166] [SPIRES].

[39] P.S. Howe, E. Sezgin and P.C. West, Covariant field equations of the M-theory five-brane, Phys. Lett. B 399 (1997) 49 [hep-th/9702008] [SPIRES].

[40] I.A. Bandos, A. Nurmagambetov and D.P. Sorokin, The type IIA NS5-brane, Nucl. Phys. B 586 (2000) 315 [hep-th/0003169] [SPIRES].

[41] M. Cederwall, B.E.W. Nilsson and P. Sundell, An action for the super-5-brane in D $=11$ supergravity, JHEP 04 (1998) 007 [hep-th/9712059] [SPIRES].

[42] E. Sezgin and P. Sundell, Aspects of the M5-brane, hep-th/9902171 [SPIRES].

[43] M. Berkooz, M. Rozali and N. Seiberg, Matrix description of M-theory on $T^{4}$ and $T^{5}$, Phys. Lett. B 408 (1997) 105 [hep-th/9704089] [SPIRES].

[44] N. Seiberg, New theories in six dimensions and matrix description of M-theory on $T^{5}$ and $T^{5} / \mathbb{Z}_{2}$, Phys. Lett. B 408 (1997) 98 [hep-th/9705221] [SPIRES].

[45] M. Bershadsky, S. Cecotti, H. Ooguri and C. Vafa, Kodaira-Spencer theory of gravity and exact results for quantum string amplitudes, Commun. Math. Phys. 165 (1994) 311 [hep-th/9309140] [SPIRES].

[46] M. Mariño, R. Minasian, G.W. Moore and A. Strominger, Nonlinear instantons from supersymmetric p-branes, JHEP 01 (2000) 005 [hep-th/9911206] [SPIRES].

[47] L. Bao, M. Cederwall and B.E.W. Nilsson, A note on topological M5-branes and string-fivebrane duality, JHEP 06 (2008) 100 [hep-th/0603120] [SPIRES].

[48] V. Bengtsson and M. Cederwall, The B-model on the A-model NS5-brane, arXiv:0805.1782 [SPIRES].

[49] M. de Vroome and S. Vandoren, Supergravity description of spacetime instantons, Class. Quant. Grav. 24 (2007) 509 [hep-th/0607055] [SPIRES].

[50] J.D. Blum and J.A. Harvey, Anomaly inflow for gauge defects, Nucl. Phys. B 416 (1994) 119 [hep-th/9310035] [SPIRES].

[51] M.J. Duff, J.T. Liu and R. Minasian, Eleven-dimensional origin of string/string duality: A one-loop test, Nucl. Phys. B 452 (1995) 261 [hep-th/9506126] [SPIRES].

[52] L. Bao, A. Kleinschmidt, B.E.W. Nilsson, D. Persson and B. Pioline, Instanton Corrections to the Universal Hypermultiplet and Automorphic Forms on $\mathrm{SU}(2,1)$, Commun. Num. Theor. Phys. 4 (2010) 187 [arXiv:0909.4299] [SPIRES].

[53] L. Bao, A. Kleinschmidt, B.E.W. Nilsson, D. Persson and B. Pioline, Rigid Calabi-Yau threefolds, Picard Eisenstein series and instantons, arXiv:1005.4848 [SPIRES].

[54] B. Pioline and D. Persson, The automorphic NS5-brane, Commun. Num. Theor. Phys. 3 (2009) 697 [arXiv:0902.3274] [SPIRES]. 
[55] P. Candelas, X.C. De La Ossa, P.S. Green and L. Parkes, A pair of Calabi-Yau manifolds as an exactly soluble superconformal theory, Nucl. Phys. B 359 (1991) 21 [SPIRES].

[56] A. Klemm and S. Theisen, Considerations of one modulus Calabi-Yau compactifications: Picard-Fuchs equations, Kähler potentials and mirror maps, Nucl. Phys. B 389 (1993) 153 [hep-th/9205041] [SPIRES].

[57] P. Candelas, X. De La Ossa, A. Font, S.H. Katz and D.R. Morrison, Mirror symmetry for two parameter models. I, Nucl. Phys. B 416 (1994) 481 [hep-th/9308083] [SPIRES].

[58] P. Candelas, A. Font, S.H. Katz and D.R. Morrison, Mirror symmetry for two parameter models. 2, Nucl. Phys. B 429 (1994) 626 [hep-th/9403187] [SPIRES].

[59] A. Kapustin, Gauge theory, topological strings and S-duality, JHEP 09 (2004) 034 [hep-th/0404041] [SPIRES].

[60] D. Maulik, N. Nekrasov, A. Okounkov and R. Pandharipande, Gromov-Witten theory and Donaldson-Thomas theory. I, Compos. Math. 142 (2006) 1263.

[61] D. Maulik, N. Nekrasov, A. Okounkov, and R. Pandharipande, Gromov-Witten theory and Donaldson-Thomas theory. II, Compos. Math. 142 (2006) 1286 [math/0406092].

[62] S. Alexandrov, B. Pioline and S. Vandoren, Self-dual Einstein Spaces, Heavenly Metrics and Twistors, J. Math. Phys. 51 (2010) 073510 [arXiv:0912.3406] [SPIRES].

[63] L. Álvarez-Gaumé, G.W. Moore, P.C. Nelson, C. Vafa and J.b. Bost, Bosonization in arbitrary genus, Phys. Lett. B 178 (1986) 41 [SPIRES].

[64] L. Álvarez-Gaumé, J.B. Bost, G.W. Moore, P.C. Nelson and C. Vafa, Bosonization on higher genus Riemann surfaces, Commun. Math. Phys. 112 (1987) 503 [SPIRES].

[65] C. Birkenhake and H. Lange, Fundamental Principles of Mathematical Sciences. Vol. 302: Complex abelian varieties, second edition, Springer-Verlag, Berlin Germany (2004).

[66] E.P. Verlinde, Attractors and the holomorphic anomaly, hep-th/0412139 [SPIRES].

[67] M. Günaydin, A. Neitzke and B. Pioline, Topological wave functions and heat equations, JHEP 12 (2006) 070 [hep-th/0607200] [SPIRES].

[68] E. Witten, Quantum background independence in string theory, hep-th/9306122 [SPIRES].

[69] M. Aganagic, V. Bouchard and A. Klemm, Topological Strings and (Almost) Modular Forms, Commun. Math. Phys. 277 (2008) 771 [hep-th/0607100] [SPIRES].

[70] A. Schwarz and X. Tang, Quantization and holomorphic anomaly, JHEP 03 (2007) 062 [hep-th/0611281] [SPIRES].

[71] I. Antoniadis, E. Gava, K.S. Narain and T.R. Taylor, Topological amplitudes in string theory, Nucl. Phys. B 413 (1994) 162 [hep-th/9307158] [SPIRES].

[72] A. Dabholkar, F. Denef, G.W. Moore and B. Pioline, Precision counting of small black holes, JHEP 10 (2005) 096 [hep-th/0507014] [SPIRES].

[73] F. Denef and G.W. Moore, Split states, entropy enigmas, holes and halos, hep-th/0702146 [SPIRES].

[74] T. Eguchi, P.B. Gilkey and A.J. Hanson, Gravitation, Gauge Theories and Differential Geometry, Phys. Rept. 66 (1980) 213 [SPIRES].

[75] S. Sethi, C. Vafa and E. Witten, Constraints on low-dimensional string compactifications, Nucl. Phys. B 480 (1996) 213 [hep-th/9606122] [SPIRES]. 
[76] I. Brunner and R. Schimmrigk, F-theory on Calabi-Yau fourfolds, Phys. Lett. B 387 (1996) 750 [hep-th/9606148] [SPIRES].

[77] J.A. Harvey and G.W. Moore, Superpotentials and membrane instantons, hep-th/9907026 [SPIRES].

[78] D. Gaiotto, G.W. Moore and A. Neitzke, Wall-crossing, Hitchin Systems and the WKB Approximation, arXiv:0907.3987 [SPIRES].

[79] E. Witten, Non-Perturbative Superpotentials In String Theory, Nucl. Phys. B 474 (1996) 343 [hep-th/9604030] [SPIRES].

[80] M. Atiyah, The logarithm of the Dedekind $\eta$-function, Math. Ann. 278 (1987) 335.

[81] S. Monnier, Geometric quantization and the metric dependence of the self-dual field, arXiv: 1011.5890 [SPIRES].

[82] A. Baarsma, J. Stienstra, T. van der Aalst and S. Vandoren, Special quaternionic manifolds from the variation of Hodge structures, in preparation.

[83] R. Minasian and G.W. Moore, K-theory and Ramond-Ramond charge, JHEP 11 (1997) 002 [hep-th/9710230] [SPIRES].

[84] M.R. Douglas, R. Reinbacher and S.-T. Yau, Branes, bundles and attractors: Bogomolov and beyond, math/0604597. MATH/0604597;

[85] S. Hosono, A. Klemm and S. Theisen, Lectures on mirror symmetry, hep-th/9403096 [SPIRES].

[86] J.M. Maldacena, A. Strominger and E. Witten, Black hole entropy in M-theory, JHEP 12 (1997) 002 [hep-th/9711053] [SPIRES].

[87] J. de Boer, M.C.N. Cheng, R. Dijkgraaf, J. Manschot and E. Verlinde, A farey tail for attractor black holes, JHEP 11 (2006) 024 [hep-th/0608059] [SPIRES].

[88] D. Gaiotto, A. Strominger and X. Yin, The M5-brane elliptic genus: Modularity and BPS states, JHEP 08 (2007) 070 [hep-th/0607010] [SPIRES].

[89] P.S. Aspinwall, D-branes on Calabi-Yau manifolds, hep-th/0403166 [SPIRES].

[90] D. Gaiotto, A. Strominger and X. Yin, New Connections Between $4 D$ and 5D Black Holes, JHEP 02 (2006) 024 [hep-th/0503217] [SPIRES].

[91] D. Gaiotto, A. Strominger and X. Yin, 5D black rings and $4 D$ black holes, JHEP 02 (2006) 023 [hep-th/0504126] [SPIRES].

[92] P. Gao and B. Pioline, Topological wave functions and the $4 D$ - 5D lift, JHEP 07 (2008) 087 [arXiv:0803.0562] [SPIRES].

[93] R. Böhm, H. Günther, C. Herrmann and J. Louis, Compactification of type IIB string theory on Calabi-Yau threefolds, Nucl. Phys. B 569 (2000) 229 [hep-th/9908007] [SPIRES].

[94] J. Manschot, On the space of elliptic genera, Commun. Num. Theor. Phys. 2 (2008) 803 [arXiv:0805.4333] [SPIRES].

[95] J. Manschot, Stability and duality in $N=2$ supergravity, Commun. Math. Phys. 299 (2010) 651 [arXiv:0906.1767] [SPIRES].

[96] M. Chiodaroli and M. Gutperle, Instantons and Wormholes in $N=2$ supergravity, Phys. Rev. D 79 (2009) 085023 [arXiv:0901.1616] [SPIRES]. 
[97] S.M. Salamon, Quaternionic Kähler manifolds, Invent. Math. 67 (1982) 143.

[98] C. LeBrun, Quaternionic-Kähler manifolds and conformal geometry, Math. Ann. 284 (1989) 353 .

[99] C. LeBrun and S. Salamon, Strong rigidity of positive quaternion-Kähler manifolds, Invent. Math. 118 (1994) 109.

[100] S. Alexandrov and P. Roche, TBA for non-perturbative moduli spaces, JHEP 06 (2010) 066 [arXiv: 1003.3964] [SPIRES].

[101] J. Manschot, Wall-crossing of D4-branes using flow trees, arXiv: 1003.1570 [SPIRES].

[102] J. Manschot, B. Pioline and A. Sen, Wall-crossing from Boltzmann black hole halos, arXiv: 1011.1258 [SPIRES].

[103] S. Alexandrov, J. Manschot and B. Pioline, S-Duality and D-Instantons, in progress.

[104] N. Nekrasov, H. Ooguri and C. Vafa, S-duality and topological strings, JHEP 10 (2004) 009 [hep-th/0403167] [SPIRES].

[105] J.A. Harvey and G.W. Moore, Exact gravitational threshold correction in the FHSV model, Phys. Rev. D 57 (1998) 2329 [hep-th/9611176] [SPIRES].

[106] E. Witten, Duality relations among topological effects in string theory, JHEP 05 (2000) 031 [hep-th/9912086] [SPIRES]. 